Oktaven und Reduktionstheorie

von Karsten Roeseler

Dissertation 



\section{Oktaven und Reduktionstheorie}

Dissertation

zur Erlangung des mathematisch-naturwissenschaftlichen Doktorgrades

„Doctor rerum naturalium“

der Georg-August-Universität zu Göttingen

vorgelegt von

Karsten Roeseler

aus Hamburg

Göttingen 2011 
Referent: Prof. Dr. Ulrich Stuhler

Korreferent: Prof. Dr. Ralf Meyer

Tag der mündlichen Prüfung: 7. Februar 2011 


\section{Inhaltsverzeichnis}

\begin{tabular}{lll}
\hline 1 & Einleitung & 3
\end{tabular}

\begin{tabular}{|ll|}
2 & Kompositionsalgebren - Oktaven \\
\hline
\end{tabular}

2.1 Definition einer Kompositionsalgebra . . . . . . . . . . . . . 5

2.2 Kompositionsalgebren über Ringen . . . . . . . . . . . . . 7

2.3 Konstruktion von Kompositionsalgebren . . . . . . . . . . . 7

2.4 Beschreibung der zerfallenden Oktav-Algebra . . . . . . . . . 10

\begin{tabular}{lll}
\hline 3 & Affine Gruppenschemata & 12
\end{tabular}

\begin{tabular}{lll}
\hline Arakelov-Bündel & 16
\end{tabular}

\begin{tabular}{|lll}
5 & Wurzelsysteme und Komplementärpolyeder & 22
\end{tabular}

$5.1 \quad$ Wurzelsysteme . . . . . . . . . . . . . . . . . 22

5.2 Ecken von Facetten . . . . . . . . . . . . . . . . . . . . 26

5.3 Reduktion und Projektion von Wurzelsystemen . . . . . . . . 27

5.4 Komplementärpolyeder. . . . . . . . . . . . . . . . . . 28

$5.5 \quad$ Stabilität und spezielle Facetten . . . . . . . . . . . . . . . 29

$\begin{array}{|lll|}6 & \text { Die Automorphismengruppe der Oktaven } & 31\end{array}$

6.1 Die Weyl-Gruppe . . . . . . . . . . . . . . . . . . 31

6.2 Bestimmung der Wurzelunterguppen . . . . . . . . . . . . . 33

$\begin{array}{lll}7 & \text { Apartments und Normen } & 40\end{array}$

7.1 Allgemeine Konstruktion eines Apartments . . . . . . . . . . 40

7.2 Die Verbindung Normen - Apartments . . . . . . . . . . . . . 43

4.3 Transfer der Wurzeluntergruppen . . . . . . . . . . . . . . . . 45

8 Weitere Strukturuntersuchungen im Normen-Gebäude 46

9 Cartan-Killing-Form und symmetrischer Raum $\quad 55$

9.1 Die Cartan-Killing-Form . . . . . . . . . . . . . . . 55

9.2 Eigenschaften der Cartan-Killing-Form . . . . . . . . . . . 60

9.3 Skalarprodukte . . . . . . . . . . . . . . . . . 60

9.4 Renormalisierung . . . . . . . . . . . . . . . . . . . 61

9.5 Ein Zusammenhang zwischen den Normen und den Einbettungen der Hamiltonschen Quaternionen . . . . . . . . . . . 62

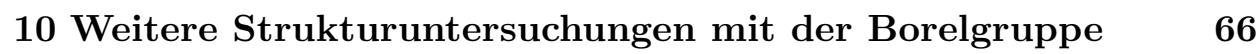

10.1 Die Filtration zur Standard-Borelgruppe . . . . . . . . . . 66

10.2 Invariante Unterräume . . . . . . . . . . . . . . . . . . 68

10.3 Versuch einer Deutung . . . . . . . . . . . . . . . . . . . 70

10.4 Algebraische Beschreibung der Flagge . . . . . . . . . . 72 
10.5 Zerfällung des Torus . . . . . . . . . . . . . . . . 74

11 Das Komplementärpolyeder für Bewertungen aus den Standardapartments

A Anhang 81

A.1 Berechnung der weiteren Schnittbedingungen aus Kapitel 8 . 81

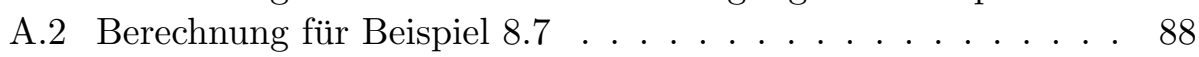
A.3 $\quad$ Berechnung der Gleichung (10.3D . . . . . . . . . . . . . . . 89

\begin{tabular}{ll}
\hline Literaturverzeichnis & 93
\end{tabular}

\begin{tabular}{ll}
\hline Index & 95
\end{tabular} 


\section{Einleitung}

"The octonions are the crazy old uncle nobody lets out of the attic."

(John C. Baez)

Oktonionen oder auch Oktaven bilden eine Kompositionsalgebra höchstmöglicher Dimension. Sie können aus einer Quaternionenalgebra ähnlich konstruiert werden wie die Quaternionenalgebra selbst aus den komplexen Zahlen. Was die Oktaven gewöhnungsbedürftig macht, ist die Eigenschaft, dass ihre Multiplikation nicht mehr assoziativ ist. Dennoch oder gerade deswegen bieten sie eine reichhaltige Grundlage für mathematische Untersuchungen.

Die vorliegende Arbeit befasst sich mit der zerfallenden Oktaven-Algebra und mit deren Automorphismengruppe $G$.

In der Hauptsache ist das Ziel der Arbeit, Untersuchungen von Behrend, Harder und Stuhler für den konkreten Fall der Gruppe $G$ zu beleuchten, die eine Lie-Gruppe vom Typ $\mathrm{G}_{2}$ ist.

Behrend hat in Beh95] das Konzept der Komplementärpolyeder eingeführt, um Instabilitätsphänomene von reduktiven Gruppenschemata über einer glatten, vollständigen algebraischen Kurve zu beschreiben. In einem Preprint HS03 haben Harder und Stuhler dieses Verfahren auf beliebige reduktive algebraische Gruppen über einem beliebigen globalen Körper verallgemeinert. Zur Beschreibung der Systeme der verschiedenen Bewertungen werden dabei symmetrische Räume für die unendlichen Primstellen sowie BruhatTits-Gebäude für die endlichen Primstellen herangezogen.

Nimmt man konkret die Automorphismengruppe der Oktaven, so gewinnt man bei Strukturuntersuchungen den Vorteil, dass hinter der Gruppenstruktur noch die Algebrenstruktur der Oktaven steht. Das legt insbesondere nahe, gruppentheoretische Beschreibungen durch algebrentheoretische zu ersetzen oder zu ergänzen. Zu einem guten Teil ist dies in dieser Arbeit gelungen: Die Beschreibung der Komplementärpolyeder zu Bewertungen im jeweiligen Standardapartment konnte auf die Grade der beiden ersten Räume in der invarianten Flagge zurückgeführt werden, die zu der Operation der zugehörigen Borelgruppe auf den Oktaven gehört. Für das Fernziel einer kompletten algebraischen Beschreibung der Komplementärpolyeder zu beliebigen kohärenten Bewertungen besteht die Hoffnung, dass mit den Ergebnissen dieser Arbeit eine gute Grundlage für weitergehende Untersuchungen gelegt wurde.

Zum Aufbau der Arbeit: In den Kapiteln 2 bis 4 werden Grundlagen über Kompositionsalgebren, affine Gruppenschemata und Arakelov-Bündel wiederholt. In Kapitel 5 wird neben der Beschreibung von Wurzelsystemen und Gewichten auch der Begriff des Komplementärpolyeders eingeführt. Es 
schließt sich in Kapitel 6 eine eingehende Beschreibung der Automorphismengruppe der Oktaven mit ihrer Struktur als reduktive Gruppe an.

Kapitel 7 und 8 beschreiben die Gebäudestruktur des Systems der Klassen von Bewertungen. Dabei wird auch darauf eingegangen, welche Schnittmenge zwei durch gewisse Elemente konjugierte Apartments haben.

Die in Kapitel 9 beschriebene Cartan-Killing-Form liefert eine mögliche Beschreibung des symmetrischen Raumes der archimedischen Bewertungen. Der Vorbereitung der schon erwähnten algebraischen Beschreibung der Komplementärpolyeder dient Kapitel 10. Ein bemerkenswertes Ergebnis ist hier die Feststellung, dass es auf den Oktaven Flaggen von Unteralgebren gibt, die unter einer gegebenen Borelgruppe invariant sind und Räume fast jeder Dimension besitzen - einzig Dimension 7 fehlt. Außerdem ergibt sich aus den Betrachtungen eine sehr prägnante Beschreibung des zu $G$ gehörenden Gebäudes.

Im letzten Kapitel wird schließlich gezeigt, dass für Bewertungen auf den jeweiligen Standardapartments das Komplementärpolyeder zu einem Punkt degradiert. Als algebraische Beschreibung wird die Lage dieses Punktes aus der invarianten Flagge heraus beschrieben und festgestellt, unter welchen Bedingungen Semistabilität im Sinne von Behrend erreicht wird.

\section{Danksagung}

Ich danke Herr Prof. Dr. Stuhler herzlich für die Themenstellung und Betreuung dieser Arbeit, für viel Geduld und viele nützliche Anregungen.

Meinen Eltern danke ich sehr für ihre in jeder Hinsicht gegebene Unterstützung während meines gesamten Studiums.

Für Korrekturlesen, technische Unterstützung und sonstigen Rat möchte ich meiner Schwester Maren, außerdem Ulrike Ober, Alexander Malinowski, Carsten Thiel und Kirstin Strokorb sowie für ganz besonderen Einsatz Kristin Stroth einen großen Dank aussprechen. 


\section{Kompositionsalgebren - Oktaven}

Grundlage für die Untersuchungen dieser Arbeit sind die 1843 zuerst von John T. Graves in einem Brief an Hamilton beschriebenen Oktaven. Ausgangspunkt war dessen Beschäftigung mit den Quaternionen. Die erste $\mathrm{Pu}-$ blikation zu den Oktaven legte jedoch Arthur Cayley 1845 mit einer von ihm selbst gefundenen Beschreibung dieser Algebra vor, so dass die Oktaven auch als Cayley-Zahlen bezeichnet werden. Mehr zur Geschichte der Konstruktion der Oktaven findet sich beispielsweise in der lesenswerten Einführung bei Baez [Bae02]. - Im Englischen scheint die Bezeichnung „octonions" weit verbreiteter als „octaves“ zu sein; im Deutschen scheint umgekehrt „Oktaven" den Vorzug vor "Oktonionen" zu genießen, und so wird es auch in der vorliegenden Arbeit gehandhabt.

In diesem Kapitel werden nun die Grundbegriffe für die Einführung der Oktaven behandelt. Für Beweise sei auf das Skript [Spr63, erster Abschnitt] von Springer bzw. das daraus weiterentwickelte Buch [SV00, Kapitel 1] von Springer und Veldkamp verwiesen.

Bei Springer (und Veldkamp) wird der Begriff der Kompositionsalgebra nur über Körpern entwickelt. Eine gewisse Erweiterung der Definition auf Ringe mit Eins ist jedoch möglich und wird im Abschnitt 2.2 behandelt.

\subsection{Definition einer Kompositionsalgebra}

Im Folgenden bezeichne $R$ immer einen kommutativen Ring mit Eins. Der Fall der Charakteristik 2 wird hier mit betrachtet, er erfordert wie an so mancher anderer Stelle einige Detailbetrachtungen.

Definition 2.1 Eine quadratische Form auf einem $R$-Modul $M$ ist eine Abbildung $N: M \rightarrow R$ mit den Eigenschaften

1. $\forall \lambda \in R, x \in M: \quad N(\lambda x)=\lambda^{2} N(x)$

2. Die Abbildung $\langle\cdot, \cdot\rangle: M \times M \rightarrow R$, die durch

$$
\langle x, y\rangle:=N(x+y)-N(x)-N(y)
$$

für $x, y \in M$ gegeben ist, ist bilinear.

$\langle\cdot, \cdot\rangle$ ist die $z u N$ assoziierte Bilinearform. Offensichtlich ist sie symmetrisch. Die Bilinearform - bzw. auch die zugehörige quadratische Form - heißt nichtausgeartet, wenn für jedes $x \in M$ gilt:

$$
(\forall y \in M:\langle x, y\rangle=0) \Rightarrow x=0 .
$$

Verallgemeinernd heißt ein Untermodul $L$ von $M$ nichtsingulär, wenn die Einschränkung der Bilinearform auf $L$ nichtausgeartet ist. 
Die Definition, wann eine quadratische Form nichtausgeartet ist, gilt hier auch im Fall der Charakteristik 2. Manche Autoren fordern abweichend davon in diesem Spezialfall eine weitere Bedingung für die Implikation, namentlich: $(N(x)=0$ und $\forall y \in M:\langle x, y\rangle=0) \Rightarrow x=0$. Für $\operatorname{char}(R) \neq 2$ sind beide Forderungen äquivalent.

Die hier nach Springer gewählte und zum Beispiel auch bei Kneser [Kne02] angewandte Definition zählt mehr Formen als ausgeartet.

Eine Kompositionsalgebra wird zunächst nur über Körpern definiert:

Definition 2.2 Eine Kompositionsalgebra $C$ über einem Körper $k$ ist eine nicht notwendigerweise assoziative $k$-Algebra mit Einselement $e$, auf der es eine nichtausgeartete quadratische Form $N$ gibt, die Komposition erlaubt, das heißt, für beliebige $x, y \in C$ gilt

$$
N(x y)=N(x) N(y) .
$$

Die quadratische Form $N$ wird dabei oft als Norm auf $C$ bezeichnet. Deren zugehörige Bilinearform $\langle\cdot, \cdot\rangle$ heißt das innere Produkt auf $C$.

Unter einer Kompositions-Unteralgebra versteht man einen nichtausgearteten Unterraum, der bezüglich der Multiplikation abgeschlossen ist und die Eins enthält.

Definition und Bemerkung 2.3 Eine wichtige Operation in einer Kompositionsalgebra ist die Konjugation. Sie ist eine Abbildung ${ }^{-}: C \rightarrow C$, definiert durch

$$
\bar{x}:=\langle x, e\rangle e-x .
$$

Die wichtigsten Rechenregeln für die Konjugation entsprechen denen der Konjugation bei den komplexen Zahlen. Insbesondere rechnet man leicht nach, dass die Konjugation eine Involution ist und dass gilt: $\forall x, y \in C$ : $\overline{x y}=\bar{y} \bar{x}$.

Diese Übereinstimmung der Rechenregeln ist insofern kein Zufall, als die komplexen Zahlen eine Kompositionsalgebra über den reellen Zahlen darstellen und die beiden Begriffe der Konjugation dann übereinstimmen.

So natürlich die Forderung nach Komposition auch erscheinen mag, so hat sie doch erhebliche Konsequenzen für die möglichen Strukturen einer Kompositionsalgebra. Zum einen äußert sich das darin, dass es bezüglich der Norm auf einer Kompositionsalgebra keine Wahlmöglichkeiten mehr gibt ganz offensichtlich muss beispielsweise $N(e)=1$ sein, und viel schärfer gilt:

Lemma 2.4 Die Norm $N$ auf einer Kompositionsalgebra ist durch die Algebrenstruktur eindeutig bestimmt.

Zum anderen ergibt die systematische Strukturuntersuchung, dass Kompositionsalgebren nicht nur von endlicher Dimension über $k$ sein müssen, sondern dass noch spezieller nur ganz bestimmte Dimensionen möglich sind: 
Satz 2.5 Eine Kompositionsalgebra $C$ über einem Körper $k$ kann nur folgende Dimensionen über $k$ haben: 1, 2, 4 und 8. Dabei kann im Fall der Charakteristik 2 die Dimension 1 nicht auftreten.

Kompositionsalgebren der Dimensionen 1 oder 2 sind kommutativ und assoziativ, solche mit Dimension 4 assoziativ, aber in keinem Fall mehr kommutativ, und solche mit Dimension 8 sind weder kommutativ noch assoziativ.

Die Erkenntnis dieses Satzes steht in direkter Verbindung mit einem Verfahren, das der Konstruktion von Kompositionsalgebren dient und im Abschnitt 2.3 erklärt wird.

\subsection{Kompositionsalgebren über Ringen}

Definition 2.6 Als eine Kompositionsalgebra $C$ über einem Ring $R$ sei eine nicht notwendigerweise assoziative $R$-Algebra mit Einselement $e$ definiert, auf der es eine nichtausgeartete quadratische Form $N$ gibt, die Komposition erlaubt, das heißt, für beliebige $x, y \in C$ gilt

$$
N(x y)=N(x) N(y)
$$

zusätzlich sei $C$ als $R$-Modul projektiv und endlich erzeugt, und für jedes maximale Ideal $\mathfrak{m}$ wird gefordert, dass die Körper-Algebra, die durch Reduktion modulo $\mathfrak{m}$ entsteht, eine Kompositionsalgebra von gleichem Rang ist.

Die Begriffe Norm, inneres Produkt und Unteralgebra übertragen sich analog aus dem Körperfall.

Wegen der geforderten guten Reduktion gilt auch bei Kompositionsalgebren über Ringen das Klassifikationsergebnis:

Satz 2.7 Eine Kompositionsalgebra $C$ über einem Ring $R$ kann nur folgende Ränge über $R$ haben: 1, 2, 4 und 8. Dabei kann im Fall der Charakteristik 2 der Rang 1 nicht auftreten.

Kompositionsalgebren vom Rang 1 oder 2 sind kommutativ und assoziativ, solche vom Rang 4 assoziativ, aber in keinem Fall mehr kommutativ, und solche vom Rang 8 sind weder kommutativ noch assoziativ.

\subsection{Konstruktion von Kompositionsalgebren}

Kompositionsalgebren können schrittweise konstruiert werden. Außer im Fall der Charakteristik 2 wird dabei mit dem Grundring begonnen. In jedem Schritt der Konstruktion wird der Rang des Moduls verdoppelt; das Verfahren heißt dementsprechend Verdopplungsverfahren beziehungsweise nach seinen Erfindern Cayley-Dickson-Verfahren. 
Satz 2.8 Sei $D$ eine Kompositionsalgebra und sei $\lambda \in R^{*}$. Auf $C:=D \oplus D$ sei über

$$
(x, y)(u, v):=(x u+\lambda \bar{v} y, v x+y \bar{u}), \quad x, y, u, v \in D
$$

ein Produkt erklärt und eine Norm durch

$$
N((x, y)):=N(x)-\lambda N(y), \quad x, y \in D
$$

definiert.

Das so erhaltene $C$ ist genau dann eine Kompositionsalgebra, wenn $D$ assoziativ ist. Außerdem ist $C$ genau dann assoziativ, wenn $D$ kommutativ und assoziativ ist.

Bemerkung 2.9 Für das Konjugieren gilt dann

$$
\overline{(x, y)}=(\bar{x},-y) \text {. }
$$

Umgekehrt kann auch jede Kompositionsalgebra durch ein solches Verfahren konstruiert werden:

Bemerkung 2.10 Innerhalb einer gegebenen Kompositionsalgebra $C$ lässt sich ein Verdopplungsverfahren durchführen, indem zu einer echten Kompositionsunteralgebra $D$ ein Element a aus $D^{\perp}$ mit $\lambda:=-N(a) \neq 0$ gewählt wird - dann ist

$$
D_{1}:=D \oplus D a
$$

eine Kompositionsunteralgebra. Für die Verknüpfungen gelten entsprechende Formeln wie in Satz2.8.

Es sei noch erwähnt, dass jedes Element $x$ einer Kompositionsalgebra folgender quadratischer Gleichung genügt:

$$
x^{2}=\langle x, e\rangle x-N(x) e .
$$

Wie man an Satz 2.7 bereits erkennen kann, ist ein Ring genau dann bereits für sich genommen eine Kompositionsalgebra (mit der identischen Abbildung als Norm), wenn nicht Charakteristik 2 vorliegt. Dies liegt an der gewählten Definition von Nichtausgeartetheit: Über einem Ring der Charakteristik 2 verschwindet das innere Produkt. Man kann aber leicht Kompositionsunteralgebren vom Rang 2 finden bzw. analog konstruieren: Wählt man ein Element $a \in C$ mit $\langle e, a\rangle \neq 0$, so ist $R e \oplus R a$ eine zweidimensionale Kompositions(unter)algebra.

Kompositionsalgebren vom Rang 2 über einem Körper $k$ sind entweder eine Körpererweiterung vom Grad 2 über $k$ oder isomorph zur direkten Summe $k \oplus k$. Letzteres ist genau dann der Fall, wenn $\mu:=\sqrt{\lambda} \in k$ ist; dann ist mit $e \mapsto(1,1)$ und $a \mapsto(\mu,-\mu)$ (mit $a$ und $\lambda$ wie in Bemerkung 2.10) ein Isomorphismus gegeben. Dass im Fall $\sqrt{\lambda} \notin k$ wieder ein Körper vorliegt, erkennt man daran, dass die Norm nicht verschwinden kann und man somit Inverse $\frac{1}{N(x)} \bar{x}$ hat. 
Definition 2.11 Eine Kompositionsalgebra vom Rang 4 über dem Grundring heißt Quaternionenalgebra, ihre Elemente sind die Quaternionen. Eine Kompositionsalgebra vom Rang 8 heißt Oktav- oder Oktonionen-Algebra, die Elemente entsprechend Oktaven oder Oktonionen.

Bei diesen höherrangigen Kompositionsalgebren ist die Klassifikation im Allgemeinen aufwendiger, es sei dafür weitgehend auf die Literatur verwiesen - bei Springer ([Spr63]/[SV00] $)$ ist dies für einige spezielle Klassen von Körpern zu finden.

Einfacher wird es im Konkreten, wenn die Algebra Nullteiler besitzt, gleichbedeutend damit, dass es $y$ ungleich null mit $N(y)=0$ gibt. Die quadratische Form $N$ ist dann also isotrop, und die Kompositionsalgebra heißt zerfallend. Es gilt in diesem Zusammenhang folgender Satz:

Satz 2.12 Für jede der Dimensionen 2, 4 und 8 gibt es bei gegebenem Körper $k$ bis auf Isomorphie genau eine zerfallende Kompositionsalgebra.

In der Dimension 4 ist das jeweils die Algebra der $2 \times 2$-Matrizen über $k$. Die Determinante stellt die Norm der Algebra dar, die Konjugation ist die Abbildung $\left(\begin{array}{ll}a & b \\ c & d\end{array}\right) \mapsto\left(\begin{array}{cc}d & -b \\ -c & a\end{array}\right)$.

Hiervon ausgehend kann man über das Verdopplungsverfahren die zerfallende Kompositionsalgebra vom Rang 8 konstruieren. Da alle zerfallenden Algebren isomorph sind, kann man $\lambda$ in Satz 2.8 beliebig wählen.

Etwas abstrakter betrachtet sind Kompositionsalgebren vom Grad 4 über einem Körper $k$ zentrale einfache Algebren und damit Azumaya-Algebren. Somit kann man auch auf die mit diesen Algebren verbundene Darstellungsweise zurückgreifen (für Körper der Charakteristik ungleich 2): Seien $r, s \in k^{*}$, und $i, j$ seien linear unabhängige Unbestimmte. Dann ist jeweils eine Kompositionsalgebra vom Rang 4 über $k$ diejenige Algebra, die von $i$ und $j$ und den folgenden Relationen erzeugt wird: $i^{2}=r, j^{2}=s, i j=-j i$. (Vergleiche hierzu zum Beispiel [Str02] oder [GS06].)

Wählt man in der eben beschriebenen Darstellung $r=s=-1$ bzw. äquivalent im Verdopplungsverfahren zweimal $\lambda=-1$ und $k=\mathbb{R}$, so erhält man den historischen Ausgangspunkt der Betrachtung solcher Algebren, die Hamiltonschen Quaternionen. Fortführend nach Dimension 8 bekommt man die sogenannten CAYleyschen ZahlenindexCayley-Zahlen im engeren Sinne. Diese sind nicht zerfallend und besitzen eine Basis aus der Eins und sieben weiteren Elementen, deren Quadrat -1 ist. Siehe hierzu zum Beispiel [Bae02], Kap. 1.

Näher an der Betrachtung von quadratischen Formen ist die folgende Darstellung einer Kompositionsalgebra: 
Lemma 2.13 Jede Oktav-Algebra über einem Körper $k$ mit char $(k) \neq 2$ hat eine Orthogonalbasis der Form e, $a, b, a b, c, a c, b c,(a b) c$ mit

$$
N(a) N(b) N(c) \neq 0
$$

Für die Kompositionsalgebren von kleinerem Rang gibt es entsprechende Basen.

\subsection{Beschreibung der zerfallenden Oktav-Algebra}

Um schließlich einmal konkret die Rechnungen in einer zerfallenden OktavAlgebra zu verdeutlichen, sei noch einmal zusammengefasst und im Detail ausgerechnet:

Bemerkung 2.14 Identifiziert man eine zerfallende Quaternionenalgebra mit einer $2 \times 2$-Matrizenalgebra, so ist die Konjugierte einer Matrix A gleich $\operatorname{det}(A) \cdot A^{-1}$, also:

$$
\overline{\left(\begin{array}{ll}
a & b \\
c & d
\end{array}\right)}=\left(\begin{array}{rr}
d & -b \\
-c & a
\end{array}\right)
$$

Die Norm auf dieser Algebra ist die Determinante.

Die Multiplikation in der daraus konstruierten Oktav-Algebra $C$ mit $\lambda=1$ stellt sich also wie folgt dar:

$$
\begin{aligned}
& \left(\left(\begin{array}{ll}
a & b \\
c & d
\end{array}\right),\left(\begin{array}{ll}
e & f \\
g & h
\end{array}\right)\right) \cdot\left(\left(\begin{array}{cc}
j & k \\
l & m
\end{array}\right),\left(\begin{array}{ll}
n & o \\
p & q
\end{array}\right)\right)= \\
& \left(\left(\begin{array}{ll}
a j+b l+q e-o g & a k+b m+q f-o h \\
c j+d l-p e+n g & c k+d m-p f+n h
\end{array}\right),\right. \\
& \left.\quad\left(\begin{array}{ll}
n a+o c+e m-f l & n b+o d-e k+f j \\
p a+q c+g m-h l & p b+q d-g k+h j
\end{array}\right)\right) .
\end{aligned}
$$

Die Konjugation in der Oktav-Algebra ist die Abbildung

$$
\left(\left(\begin{array}{ll}
a & b \\
c & d
\end{array}\right),\left(\begin{array}{ll}
e & f \\
g & h
\end{array}\right)\right) \mapsto \quad\left(\left(\begin{array}{rr}
d & -b \\
-c & a
\end{array}\right),\left(\begin{array}{ll}
-e & -f \\
-g & -h
\end{array}\right)\right) .
$$

Die Norm einer Oktave $(A, B)$ ist

$$
N((A, B))=\operatorname{det}(A)-\operatorname{det}(B) .
$$

Definition 2.15 Im weiteren Verlauf dieser Arbeit wird immer auf diese Darstellung der Oktaven $C$ Bezug genommen. Es sei dafür

$$
\boldsymbol{a}:=\left(\left(\begin{array}{ll}
0 & 0 \\
0 & 0
\end{array}\right),\left(\begin{array}{ll}
1 & 0 \\
0 & 1
\end{array}\right)\right)
$$


festgelegt, also

$$
\left(\left(\begin{array}{ll}
a & b \\
c & d
\end{array}\right),\left(\begin{array}{ll}
e & f \\
g & h
\end{array}\right)\right)=\left(\begin{array}{ll}
a & b \\
c & d
\end{array}\right)+\left(\begin{array}{ll}
e & f \\
g & h
\end{array}\right) \boldsymbol{a} .
$$

Mit dem Buchstaben $C$ wird im Folgenden nur noch eine Oktav-Algebra über einem Ring $R$ bezeichnet. Es ist also

$$
C=\mathrm{M}_{2}(R) \oplus \mathrm{M}_{2}(R) \boldsymbol{a} .
$$

Bemerkung 2.16 Wegen $\operatorname{det}(A+E)=\operatorname{det}(A)+\operatorname{Spur}(A)+1$ für eine $2 \times 2$ Matrix A mit Einheitsmatrix E stellt sich die quadratische Gleichung 2.1 für eine Oktave $x=(A, B)$ in dieser Form dar:

$$
x^{2}=\operatorname{Spur}(A) x-N(x) e .
$$




\section{Affine Gruppenschemata}

Affine Gruppenschemata sind bestimmte gruppenwertige Funktoren auf Ringen. Von solch einem Funktor wird verlangt, dass er darstellbar ist - siehe dazu in Satz 3.1.

Sehr prominente Beispiele sind die Matrixgruppen $\mathrm{GL}_{n}(R)$ oder $\mathrm{SL}_{n}(R)$, die über einem Ring $R$ gebildet werden.

Mit $r$ sei in diesem Kapitel ein Grundring von Konstanten bezeichnet, über dem die behandelten Objekte definiert sein sollen. Weiterhin werden mit $R$ kommutative Ringe mit Eins bezeichnet, die ebenfalls über $r$ definiert sein sollen und somit als $r$-Algebren aufgefasst werden können. (Injektivität der Abbildung $r \rightarrow R$ wird im Allgemeinen nicht gefordert.) Sei $r$-ALG die Kategorie der $r$-Algebren, GrP die der Gruppen.

Affine Gruppenschemata sind anschaulich beschrieben aus Ringen $R$ gebildete Gruppen, die durch Gleichungen bestimmt werden. Weil diese Beschreibung in direkter Form jedoch schwer zu fassen ist, wird oftmals auf die zu den Gruppen dualen Algebren übergegangen.

Satz 3.1 (nach [Wat79], Abschnitt 1.2) Sei F ein Funktor von $r$-ALG nach GrP. Wenn die Elemente von $F(R)$ mit den in $R$ liegenden Lösungen einer Familie von Gleichungen korrespondieren, dann gibt es eine $r$-Algebra $A$ mit einer natürlichen Entsprechung zwischen $F(R)$ und $\operatorname{Hom}_{r}(A, R)$. Die Umkehrung gilt ebenfalls.

Definition 3.2 Ein Funktor $F$, der die Bedingung aus dem vorigen Satz erfüllt, heißt darstellbar, und man sagt, dass $F$ von $A$ dargestellt wird.

Bei Gruppen wie $\mathrm{SL}_{n}(R)$ oder $\mathrm{O}_{n}(R)$ ist klar, dass die entsprechenden Funktoren darstellbar sind, da die Definition auf ein Gleichungssystem in den Matrixeinträgen führt.

Invertierbarkeitsbedingungen wie bei $\mathrm{GL}_{n}(R)$ können ebenfalls zu einer Gleichung gemacht werden, indem man einen zusätzlichen Parameter $z$ einführt und fordert, dass das Produkt aus dem zu Invertierenden und $z$ gleich 1 wird.

Eine ganz allgemeine Lösung einer Familie von Gleichungen erhält man, indem man die Unbestimmten der Gleichungen als Unbestimmte in einem Polynomring über $r$ heranzieht und die gegebenen Gleichungen als Relationen herausteilt; für die $\mathrm{SL}_{n}(R)$ ergibt dies beispielsweise den Ring $A=r\left[X_{11}, X_{12}, X_{21}, X_{22}\right] /\left(X_{11} X_{22}-X_{12} X_{21}-1\right)$. Jede spezielle Lösung der Gleichungsfamilie entspricht dann einem Homomorphismus des konstruierten Quotientenrings in den Ring $R$ - das ist die in Satz 3.1 genannte Entsprechung von $F(R)$ und $\operatorname{Hom}_{r}(A, R)$.

Definition 3.3 Ein affines Gruppenschema über $r$ ist ein darstellbarer Funktor von $r$-ALG nach GRP. 
Bemerkung 3.4 Die in der algebraischen Geometrie betrachteten Schemata sind Verallgemeinerungen des hier definierten Begriffs. Der Zusatz „affin" drückt aus, dass die Mengen mit Lösungen von Polynomen in Zusammenhang stehen.

Diesem Zusammenhang zur algebraischen Geometrie entsprechend hat man auf den $r$-Algebren, die die Funktoren im obigen Sinne darstellen, eine Kostruktur.

Seien $E$ und $F$ zwei darstellbare Funktoren, die durch $A$ bzw. $B$ dargestellt werden. Der Produktfunktor

$$
(E \times F)(R)=\{(e, f) \mid e \in E(R), f \in F(R)\}
$$

wird dann durch $A \otimes_{r} B$ dargestellt, denn die Homomorphismen von $A \otimes B$ nach $R$ entsprechen genau den Paaren von Homomorphismen von $A$ und $B$ nach $R$.

Der Funktor, der jede $r$-Algebra $R$ auf die einelementige Gruppe abbildet, wird durch $r$ dargestellt.

Zur Übersetzung der Strukturen - Gruppenstruktur auf der einen Seite, Kostruktur auf der Algebren-Seite - ist das folgende Yoneda-Lemma hilfreich (zitiert nach [Wat79], Abschnitt 1.3). Dazu wird definiert, dass eine Abbildung zwischen Funktoren $E$ und $F$ natürlich heißt, wenn für jeden Morphismus $\varphi: R \rightarrow S$ das Diagramm

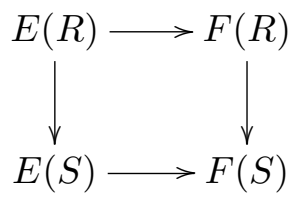

kommutiert.

Lemma 3.5 (Yoneda) Seien $E$ und F Funktoren, die durch die $r$-Algebren $A$ bzw. $B$ dargestellt werden. Die natürlichen Abbildungen $E \rightarrow F$ entsprechen dann den $r$-Algebrenhomomorphismen $B \rightarrow A$.

Zunächst wird noch eine kategorientheoretische Beschreibung einer Gruppe benötigt.

Eine Gruppe ist eine Menge $\Gamma$ zusammen mit Abbildungen

$$
\begin{array}{rlrl} 
& \text { mult }: & \Gamma \times \Gamma & \rightarrow \Gamma \\
\text { unit : } & & \{e\} & \rightarrow \Gamma \\
\text { inv : } & \Gamma & \rightarrow \Gamma
\end{array}
$$


und der Eigenschaft, dass die folgenden Diagramme kommutieren:

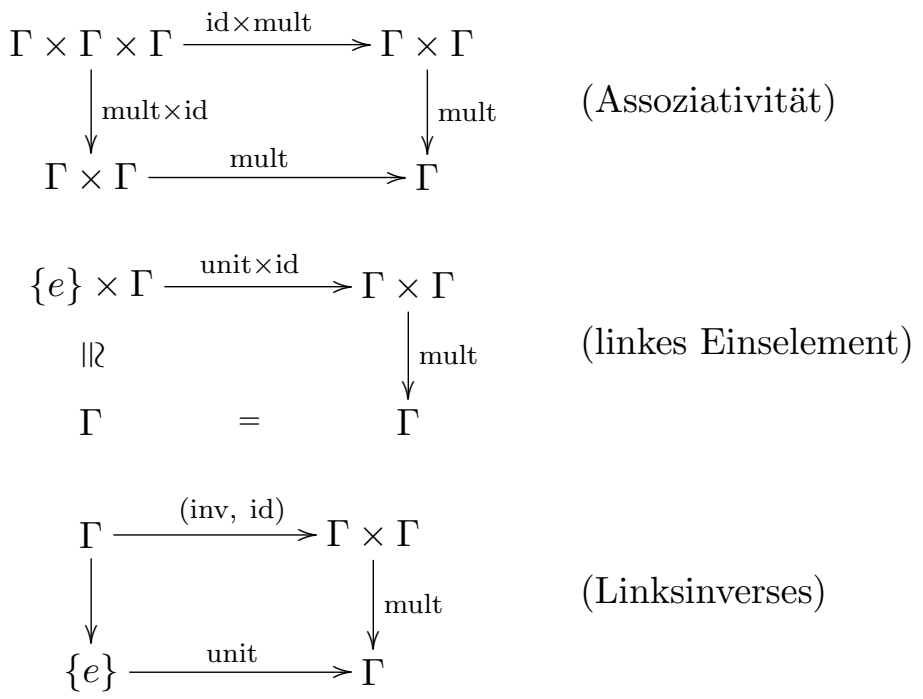

Wenn $G$ ein Gruppenfunktor und $R \rightarrow S$ ein Algebrenhomomorphismus ist, dann ist die induzierte Abbildung $G(R) \rightarrow G(S)$ ein Homomorphismus; das heißt, dass das Diagramm

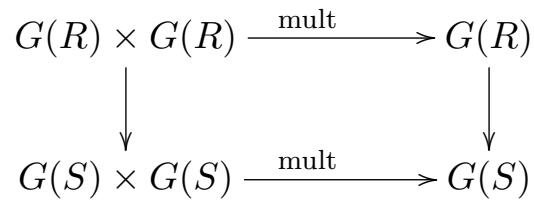

kommutiert. Dies wiederum besagt nichts anderes, als dass die Abbildung mult eine natürliche Abbildung ist. Auf ähnliche Weise kann man zeigen, dass auch unit und inv natürliche Abbildungen sind.

Nun kann auf diese Abbildungen das Yoneda-Lemma angewandt werden. Man erhält daraus die dualen Abbildungen

$$
\begin{aligned}
& \Delta: A \rightarrow A \otimes A \quad \text { Komultiplikation, } \\
& \varepsilon: A \rightarrow r \quad \text { Koeins bzw. Vergrößerung, } \\
& S: A \rightarrow A \quad \text { Koinverses (Antipode) }
\end{aligned}
$$

und die Kommutativität der folgenden Diagramme in der dualen Kategorie: 


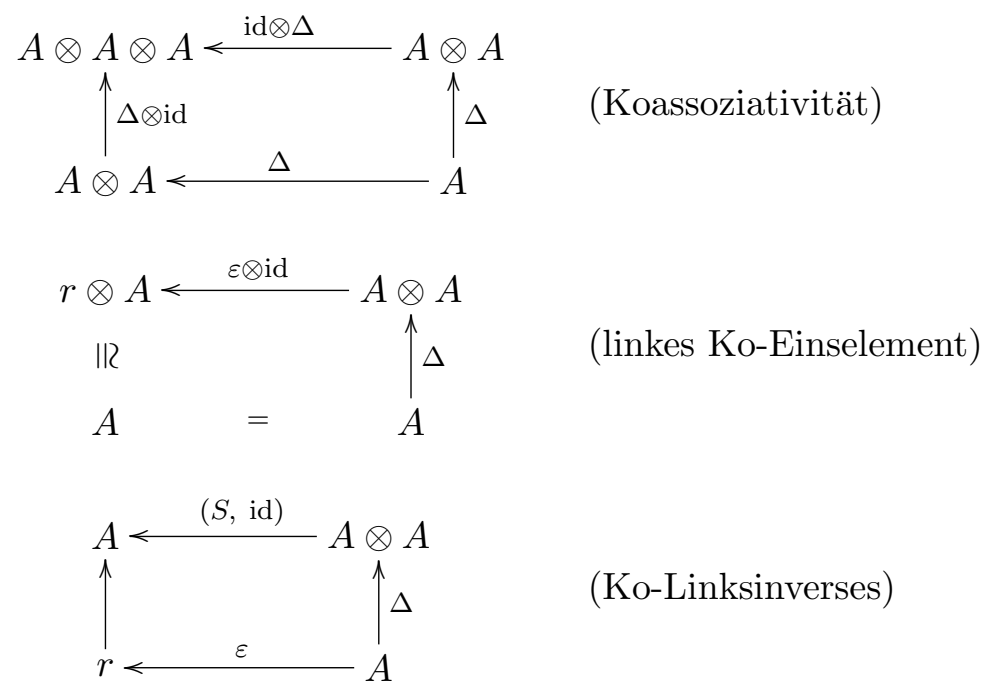

Eine $r$-Algebra mit der Angabe von Abbildungen $\Delta, \varepsilon$ und $S$, die diese Bedingungen erfüllen, heißt Hopf-Algebra.

Satz 3.6 Affine Gruppenschemata über r korrespondieren zu Hopf-Algebren über $r$.

In dieser Arbeit wird als affines Gruppenschema vor allem die Automorphismengruppe der in Kapitel 2 definierten Oktaven betrachtet. Die Darstellbarkeit des entsprechenden Funktors soll hier kurz gezeigt werden.

Dazu seien $C$ die über $r$ definierten zerfallenden Oktaven und $R$ eine $r$ Algebra. Man betrachte die Konstruktion von $C \otimes_{r} R$ nach dem Verdopplungsverfahren als $\mathrm{M}_{2}(r) \otimes_{r} R \oplus \mathrm{M}_{2}(r) \otimes_{r} R$. Mit der Wahl $\lambda=1$ ergibt sich wie schon in Abschnitt 2.4 beschrieben die Multiplikationsvorschrift $(x, y)(u, v)=(x u+\bar{v} y, v x+y \bar{u})$. Das Dualisieren $z \mapsto \bar{z}$ einer Matrix geschieht hierbei durch $\left(\begin{array}{ll}a & b \\ c & d\end{array}\right) \mapsto\left(\begin{array}{cc}d & -b \\ -c & a\end{array}\right)$, und für invertierbare $z$ ist also $z \bar{z}=\operatorname{det}(z)$.

$C \otimes_{r} R$ ist ein 8-dimensionaler freier $R$-Modul, seine Automorphismengruppe daher eine Untergruppe der $\mathrm{GL}_{8}(R)=\mathrm{GL}_{8}(r) \otimes_{r} R$. Die Forderung, dass ein Automorphismus $\alpha \in \operatorname{Aut}(C \otimes R)$ mit der Multiplikation verträglich ist, führt über den Koeffizientenvergleich auf polynomiale Gleichungen in den Einträgen der Matrix $\alpha \in \mathrm{GL}_{8}(r) \otimes R$, ebenso gilt dies für die Forderung nach Verträglichkeit mit der Dualisierung.

Korollar 3.7 Damit ist $R \mapsto \operatorname{Aut}_{R}(C \otimes R)$ ein darstellbarer Funktor und somit ein affines Gruppenschema. 


\section{Arakelov-Bündel}

Der Inhalt dieses Kapitels folgt dem ersten Kapitel der Arbeit [HS03] von Harder und Stuhler. Dort finden sich auch Angaben zu den nicht aufgeführten Beweisen.

Grundlegende Notationen und Definitionen. Im Folgenden sei mit $K$ immer ein globaler Körper bezeichnet, das heißt ein Zahlkörper oder ein Körper algebraischer Funktionen von Transzendenzgrad 1 über einem endlichen Körper von Konstanten.

$X$ bezeichne die Menge der Äquivalenzklassen von Beträgen auf $K$. Darin eingeschlossen sei im Fall eines Zahlkörpers die endliche Menge $X_{\infty}$ an archimedischen Beträgen.

Die Elemente von $X$ werden üblicherweise mit $|\cdot|_{v}$ bzw. vereinfachend $v$ oder $|\cdot|$ bezeichnet.

Für beliebiges $v \in X$ sei $K_{v}$ eine Vervollständigung von $K$ an der Stelle $v$. In dem Fall, dass $v$ eine nicht-archimedische Primstelle ist, wird der zugehörige diskrete Bewertungsring mit $\mathcal{O}_{v}=\left\{\left.\lambda \in K_{v}|| \lambda\right|_{v} \leq 1\right\}$ bezeichnet.

Die möglichen Normierungen der Beträge $v \in X$ seien so gewählt, dass die Produktformel

$$
\prod_{v \in X}|\lambda|_{v}=1
$$

für alle $\lambda \in K, \lambda \neq 0$ gilt.

Definition 4.1 Ein normierter $K_{v}$-Vektorraum $(V,\|\cdot\|)$ ist ein endlichdimensionaler $K_{v}$-Vektorraum zusammen mit einer reellwertigen Norm

$$
\|\cdot\|: V \rightarrow \mathbb{R}, \quad a \mapsto\|a\|,
$$

die die folgenden Bedingungen erfüllt:

1. $\forall a \in V:\|a\| \geq 0$ und $\|a\|=0 \Leftrightarrow a=0$,

2. $\forall \lambda \in K_{v}, a \in V:\|\lambda a\|=|\lambda|_{v}\|a\|$,

3. (a) Falls $v \in X^{(\infty)}:=X \backslash X_{\infty}$, d. h. eine nicht-archimedische Primstelle ist, gelte für alle $a_{1}, a_{2} \in V$ :

$$
\left\|a_{1}+a_{2}\right\| \leq \max \left\{\left\|a_{1}\right\|,\left\|a_{2}\right\|\right\} .
$$

(b) Falls $v \in X_{\infty}$, also eine archimedische Primstelle ist, gelte für alle $a_{1}, a_{2} \in V$

$$
\left\|a_{1}+a_{2}\right\| \leq\left\|a_{1}\right\|+\left\|a_{2}\right\| .
$$

Zur eindeutigen Unterscheidung wird gegebenenfalls die Norm mit $\|\cdot\|_{v}$ bezeichnet. 
Definition 4.2 Sei $v \in X^{(\infty)}$ eine nicht-archimedische Primstelle, sei $V$ ein $K_{v^{-}}$Vektorraum und $E \subset V$ ein $\mathcal{O}_{v}$-Gitter in $V$, das heißt, $E$ ist ein freier $\mathcal{O}_{v}$-Modul von endlichem Rang so, dass $K_{v} \cdot E=V$ ist.

Dann wird für $a \in V, a \neq 0$ definiert:

$$
\|a\|:=\inf \left\{|\lambda|_{v} \mid \lambda \in K_{v}^{*}, \lambda^{-1} a \in E\right\},
$$

zudem selbstverständlich $\|0\|:=0$.

Auf diese Weise erhält man eine Norm auf $V$, die so gewählt ist, dass $E$ gleich der Einheitskugel

$$
\{a \in V \mid\|a\| \leq 1\}
$$

von $V$ ist.

\section{Bemerkungen 4.3}

- Für $K=\mathbb{Q}, V=K^{n}$ und den üblichen Absolutbetrag $|\cdot|_{p}$ bezüglich einer Primzahl $p$ ist

$$
\|a\|_{p}=\left\|\left(a_{1}, a_{2}, \ldots, a_{n}\right)\right\|_{p}=\max _{i=1, \ldots, n}\left|a_{i}\right|_{p} .
$$

- Im Fall, dass $v \in X_{\infty}$ eine archimedische Primstelle ist, folgt aus den Bedingungen 2. und 3. von Definition 4.1 insbesondere, dass die Einheitskugel $\{a \in V:\|a\| \leq 1\}$ eine konvexe Teilmenge von $V$ ist.

- Normen zu archimedischen Primstellen werden im späteren Verlauf dieser Arbeit meist durch ein reelles oder hermitesches Skalarprodukt $(\cdot, \cdot)$ so gegeben, dass $\|a\|=(a, a)^{\frac{1}{2}}$ für $a \in V$ ist.

Definition 4.4 Sei $V$ ein endlich-dimensionaler Vektorraum über dem globalen Körper $K$. Eine Menge von Normen

$$
\left\{\|\cdot\|_{v}: K_{v} \otimes_{K} V \rightarrow \mathbb{R} \mid v \in X\right\}
$$

heiße kohärent, wenn es eine ko-endliche Teilmenge $X^{\prime} \subseteq X^{(\infty)}$, einen freien Modul $E$ über dem Ring der ganzen Zahlen $\mathcal{O}\left(X^{\prime}\right):=\left\{\left.\lambda \in K|| \lambda\right|_{v} \leq\right.$ $\left.1 \forall v \in X^{\prime}\right\}$ und einen $K$-Vektorraum-Isomorphismus $\varphi: K \otimes_{\mathcal{O}\left(X^{\prime}\right)} E \stackrel{\sim}{\rightarrow} V$ so gibt, dass die Normen $\|\cdot\|_{v}$ für $v \in X^{\prime}$ durch die Gitter

$$
\mathcal{O}_{v} \otimes_{\mathcal{O}\left(X^{\prime}\right)} E \hookrightarrow V_{v}:=K_{v} \otimes_{K} V
$$

gegeben sind, wobei die Einbettungen durch die von $\varphi$ induzierten Isomorphismen

$$
\operatorname{id}_{K_{v}} \otimes \varphi: K_{v} \otimes_{\mathcal{O}\left(X^{\prime}\right)} E \stackrel{\sim}{\rightarrow} V_{v}=K_{v} \otimes_{K} V
$$

gegeben sind.

Bemerkung 4.5 Selbstverständlich ist $X^{\prime}$ durch die obigen Bedingungen nicht eindeutig definiert, es kann zum Beispiel durch eine kleinere ko-endliche Menge $X^{\prime \prime} \subset X^{\prime}$ ersetzt werden. 
Definition 4.6 Ein Arakelov-Bündel $E=\left(V ;\|\cdot\|_{v}\right.$ für $\left.v \in X\right)$ vom Rang $d$ über $K$ besteht aus einem $d$-dimensionalen $K$-Vektorraum $V$ und einer kohärenten Menge von Normen $\left\{\|\cdot\|_{v} \mid v \in X\right\}$ auf den $K_{v}$-Vektorräumen $K_{v} \otimes_{K} V$, wobei zusätzlich angenommen wird, dass für jedes $v \in X_{\infty}$ die Norm $\|\cdot\|_{v}$ durch ein Skalarprodukt $(\cdot, \cdot)_{v}$ auf $V_{v}$ gegeben ist.

\section{Bemerkungen 4.7}

1. Falls $X^{\prime} \subseteq X^{(\infty)}$ ko-endlich ist, $\mathcal{O}\left(X^{\prime}\right)=\left\{\left.\lambda \in K|| \lambda\right|_{v} \leq 1 \forall v \in\right.$ $\left.X^{\prime}\right\}$ gilt und $M$ ein freier oder noch allgemeiner projektiver Modul vom Rang d über $\mathcal{O}\left(X^{\prime}\right)$ ist, ist ein Arakelov-Bündel über $K$ bereits charakterisiert durch die Daten $\left(M ;\|\cdot\|_{v}\right.$ für $\left.v \in X \backslash X^{\prime}\right)$, wobei $\|\cdot\|_{v}$ Normen auf $K_{v} \otimes_{\mathcal{O}\left(X^{\prime}\right)} M$ sind.

2. Für ein gegebenes Arakelov-Bündel $E=\left(V ;\|\cdot\|_{v}\right.$ für $\left.v \in X\right)$ sei als $E_{\eta}:=V$ die generische Faser, also die Faser des Arakelov-Bündels $E$ an der generischen Primstelle bezeichnet.

Definition 4.8 Ein Homomorphismus $\varphi: E_{1} \rightarrow E_{2}$ zwischen ArakelovBündeln $E_{i}=\left(V_{i} ;\|\cdot\|_{v}^{(i)}\right.$ für $\left.v \in X\right), i=1,2$ ist eine $K$-lineare Abbildung $\varphi_{\eta}: V_{1} \rightarrow V_{2}$, die für alle $v \in X, a \in V_{1, v}$ der Ungleichung

$$
\left\|\left(\operatorname{id}_{K_{v}} \otimes_{K} \varphi_{\eta}\right)(a)\right\|_{v}^{(2)} \leq\|a\|_{v}^{(1)}
$$

genügt.

Bemerkung 4.9 Sofern das eben definierte $\varphi_{\eta}$ ein Isomorphismus ist und die $\varphi_{v}:=\operatorname{id}_{K_{v}} \otimes_{K} \varphi_{\eta}$ normerhaltend sind - das heißt, dass bei (4.2) Gleichheit gilt -, dann ist $\varphi$ ein Isomorphismus in der Kategorie der ArakelovBündel.

Beweis. $\varphi_{\eta}^{-1}$ induziert einen Umkehrmorphismus.

Bemerkung 4.10 Wenn die Arakelov-Bündel $E_{i}$ durch $\left(M_{i} ;\|\cdot\|_{v}^{(i)}\right.$ für $v \in$ $S), i=1,2$ im Sinne von Teil 1 der Bemerkungen 4.7 gegeben sind, kann ein Homomorphismus $\varphi: E_{1} \rightarrow E_{2}$ durch einen $\mathcal{O}$-Modul-Homomorphismus $\varphi: M_{1} \rightarrow M_{2}$ gegeben werden, der die Kontraktionseigenschaft von Definition 4.8 für $v \in S$ erfüllt.

\section{Definition 4.11}

1. Ein Arakelov-Bündel $E=\left(V ;\|\cdot\|_{v}\right.$ für $\left.v \in X\right)$ von Rang 1, das heißt mit $\operatorname{dim}_{K}(V)=1$, wird auch Arakelov-Geradenbündel genannt.

2. Angenommen, es sei $d=\operatorname{rang}(E)=\operatorname{dim}_{K}(V)$. Dann heißt die $d$-te äußere Potenz

$$
\Lambda^{d}(E):=\left(\Lambda^{d}(V) ; \Lambda^{d}\left(\|\cdot\|_{v}\right) \text { für } v \in X\right)
$$

das Determinanten-Geradenbündel von E. Es ist ein Arakelov-Geradenbündel, das oft als $\operatorname{det}(E)$ bezeichnet wird. 
Bemerkung 4.12 Auf ähnliche Weise hat man die üblichen Tensor-Konstruktionen in der Kategorie der Arakelov-Bündel, insbesondere das Tensorprodukt $E_{1} \otimes E_{2}$ und das Hom-Bündel $\underline{\operatorname{Hom}}\left(E_{1}, E_{2}\right)$.

\section{Definition und Satz 4.13}

1. Wenn $E=\left(V ;\|\cdot\|_{v}\right.$ für $\left.v \in X\right)$ ein Arakelov-Bündel ist, hat man für jeden $K$-linearen Unterraum $V_{1} \subset V$ das Unterbündel

$$
\left(V_{1} ; \|\left.\cdot||_{v}\right|_{K_{v} \otimes V_{1}} \text { für } v \in X\right) .
$$

Die Inklusionsabbildung $i: V_{1} \hookrightarrow V$ induziert einen Homomorphismus von Arakelov-Bündeln.

2. Dual betrachtet erhält der Quotientenvektorraum $V_{2}:=V / V_{1}$ Normen auf $K_{v} \otimes_{K} V_{2}$ für $v \in X$ wie folgt: Es sei $\bar{a}=a+V_{1} \in V / V_{1}$, dann definiert man:

$$
\begin{aligned}
\|\bar{a}\|_{v}^{(2)} & :=\inf \left\{\left\|a+a_{1}\right\|_{v} \mid a_{1} \in V_{1, v}\right\} \\
E / E_{1} & :=\left(V / V_{1} ;\|\cdot\|_{v}^{(2)} \text { für } v \in X\right) .
\end{aligned}
$$

Damit ist dann $E / E_{1}$ ein Arakelov-Bündel, das das Quotientenbündel von $E$ durch $E_{1}$ genannt wird. Die Projektion $p: V \rightarrow V / V_{1}$ induziert einen Homomorphismus $p: E \rightarrow E / E_{1}=: E_{2}$ von Arakelov-Bündeln.

Der Beweis ist klar.

\section{Definition und Bemerkung 4.14 Man betrachte}

$$
0 \rightarrow E_{1} \rightarrow E \rightarrow E_{2}=E / E_{1} \rightarrow 0
$$

als eine exakte Sequenz in der Kategorie von Arakelov-Bündeln. Jede Sequenz, die isomorph zu dieser Sequenz ist, wird exakt genannt. Anders als im Funktionenkörperfall ist die Kategorie der Arakelov-Bündel im Allgemeinen jedoch nicht mehr additiv.

Definition 4.15 Wie schon erwähnt seien die Betragsfunktionen $|\cdot|_{v}, v \in$ $X$ so gewählt, dass die Produktbeziehung $\prod_{v \in X}|\lambda|_{v}=1$ für jedes von null verschiedene Element $\lambda \in K$ gilt.

1. Es sei $L=\left(V ;\|\cdot\|_{v}\right.$ für $\left.v \in X\right)$ ein Arakelov-Geradenbündel auf $X$. Wenn $a \in V$ ein beliebiges von null verschiedenes Element von $V$ ist, wird der Grad von $L$ definiert als

$$
\operatorname{deg}(L):=-\sum_{v \in X} \log \|a\|_{v},
$$

er ist also im Allgemeinen eine reelle Zahl. Der Wert des Grades ist dabei unabhängig von der Wahl von $a$. 
2. Für ein Arakelov-Bündel $E$ von beliebigem Rang definiert man

$$
\operatorname{deg}(E):=\operatorname{deg}(\operatorname{det}(E)) .
$$

Die folgenden Sätze werden in dieser Arbeit nicht benötigt, werden aber mit höchster Wahrscheinlichkeit bei sich anschließenden Untersuchungen von Bedeutung sein. Denn bei den Gittern in den Gebäuden der Normen, die außerhalb des Standardgitters liegen, müssen die komplizierteren Strukturen beachtet werden.

\section{Satz 4.16}

1. Seien $L_{1}$ und $L_{2}$ Arakelov-Geradenbündel. Dann gilt:

$$
\operatorname{deg}\left(L_{1} \otimes L_{2}\right)=\operatorname{deg}\left(L_{1}\right)+\operatorname{deg}\left(L_{2}\right) .
$$

2. $S e i$

$$
0 \rightarrow E_{1} \rightarrow E \rightarrow E_{2} \rightarrow 0
$$

eine exakte Sequenz von Arakelov-Bündeln. Dann gibt es eine kanonische Isomorphie

$$
\operatorname{det}\left(E_{1}\right) \otimes \operatorname{det}\left(E_{2}\right) \stackrel{\sim}{\rightarrow} \operatorname{det}(E)
$$

von Geradenbündeln.

Insbesondere gilt:

$$
\operatorname{deg}(E)=\operatorname{deg}\left(E_{1}\right)+\operatorname{deg}\left(E_{2}\right)
$$

Definition 4.17 Sei $E$ ein nichttriviales Arakelov-Bündel. Dann definiert man als

$$
\mu(E):=\frac{\operatorname{deg}(E)}{\operatorname{rang}(E)}
$$

die Steigung des Arakelov-Bündels E.

Definition 4.18 Ein Arakelov-Bündel $E$ heißt stabil (semistabil), wenn für alle von Null verschiedenen Unterbündel $E_{1} \subsetneq E$ die Ungleichung

$$
\mu\left(E_{1}\right)<\mu(E) \quad\left(\text { bzw. } \quad \mu\left(E_{1}\right) \leq \mu(E)\right)
$$

gilt.

Satz 4.19 Ein Arakelov-Bündel E hat eine eindeutige Flagge von Unterbündeln

$$
0=E_{0} \subsetneq E_{1} \subsetneq \ldots \subsetneq E_{r}=E,
$$

die die folgenden Eigenschaften erfüllt: 
1. $E_{i} / E_{i-1}$ ist für jedes $i$ semistabil.

2. Es gilt $\mu\left(E_{i} / E_{i-1}\right)>\mu\left(E_{i+1} / E_{i}\right)$ für jedes $i$.

Diese Flagge heißt Harder-Narasimhan-Filtration, abgekürzt HN-Filtration. Die Filtration erfüllt zudem Folgendes:

3. $E_{i} / E_{i-1}$ ist das größte Unterbündel von $E / E_{i-1}$ mit der Eigenschaft, dass

$$
\mu\left(E_{i} / E_{i-1}\right)=\mu_{\max }\left(E / E_{i-1}\right)
$$

gilt. Dabei sei mit $\mu_{\max }\left(E / E_{i-1}\right)$ der größte vorkommende Wert von $\mu$ für alle Unterbündel von $E / E_{i-1}$ bezeichnet.

4. $E_{i} / E_{i-1}$ ist das kleinste Quotientenbündel von $E_{i}$ mit der Eigenschaft, dass

$$
\mu\left(E_{i} / E_{i-1}\right)=\mu_{\min }\left(E_{i}\right)
$$

gilt. Dabei sei mit $\mu_{\min }\left(E_{i}\right)$ der kleinste vorkommende Wert von $\mu$ für alle Quotienten von $E_{i}$ bezeichnet.

Satz 4.20 Sei $E^{\prime} \subset E$ ein Unterbündel eines Arakelov-Bündels E. Sei zudem $E_{1}^{\prime} \subset \ldots \subset E_{r}^{\prime}$ die $H N$-Filtration von $E^{\prime}$ und

$$
E_{1} / E^{\prime} \subset \ldots \subset E_{s-1} / E^{\prime}
$$

die HN-Filtration des Bündels E/E'. Schließlich sei noch die Gültigkeit von

$$
\mu_{\max }\left(E / E^{\prime}\right)<\mu_{\min }\left(E^{\prime}\right)
$$

vorausgesetzt. Dann ist die HN-Filtration von E die Flagge

$$
0 \subset E_{1}^{\prime} \subset \ldots \subset E_{r-1}^{\prime} \subset E^{\prime} \subset E_{1} \subset \ldots \subset E_{s-1} \subset E .
$$

Insbesondere ist $E^{\prime}$ ein Teil der kanonischen Filtration von E. 


\section{Wurzelsysteme und Komplementärpolyeder}

\subsection{Wurzelsysteme}

Der Text dieses Abschnitts folgt in weiten Teilen den Einführungen von [HS03, Abschnitt 2] und [Beh95, Abschnitt 1], ergänzt durch eigene Zusätze. Nicht ausgeführte Beweise finden sich in der angegebenen Literatur.

Definition 5.1 Sei $V$ ein reeller Vektorraum und $V^{*}$ sein Dualraum. Ein Wurzelsystem $\Phi$ ist eine Teilmenge von $V$ - die Elemente von $\Phi$ heißen Wurzeln - mit den folgenden Eigenschaften:

1. Die Menge $\Phi$ ist endlich und erzeugt $V$.

2. Für jede Wurzel $\alpha \in \Phi$ gibt es ein eindeutig bestimmtes Element $\check{\alpha} \in V^{*}$ derart, dass mit der kanonischen Paarung $\langle\cdot, \cdot\rangle$ von $V$ und seinem Dualraum die Gleichheit

$$
\langle\alpha, \check{\alpha}\rangle=2
$$

gilt und dass für die zu $\alpha$ gehörige Spiegelung

$$
s_{\alpha}: V \rightarrow V, x \mapsto s_{\alpha}(x):=x-\langle x, \check{\alpha}\rangle \alpha
$$

die Beziehung $s_{\alpha}(\Phi)=\Phi$ gilt.

3. Für jede Wurzel $\alpha \in \Phi$ ist $\langle\Phi, \check{\alpha}\rangle \subseteq \mathbb{Z}$.

Ein Wurzelsystem $\Phi$ heißt reduziert, wenn außerdem gilt:

4. Für alle $\alpha \in \Phi$ ist $2 \alpha \notin \Phi$.

Die Menge der Kowurzeln $\check{\alpha}$ wird analog mit $\Phi^{\vee}$ bezeichnet. Sie ist ein Wurzelsystem in $V^{*}$.

Definition 5.2 Für $\alpha \in \Phi$ definiert man die Hyperebene

$$
H(\alpha):=\{x \in V \mid\langle x, \check{\alpha}\rangle=0\} .
$$

Die Menge $\{H(\alpha) \mid \alpha \in \Phi\}$ liefert die bekannte Zerlegung von $V$ in Facetten: in Formeln ausgedrückt liegen zwei Elemente $x, y \in V$ genau dann in derselben Facette, wenn für alle $\alpha \in \Phi$ gilt:

$$
\langle x, \check{\alpha}\rangle \lesseqgtr 0 \quad \Longleftrightarrow\langle y, \check{\alpha}\rangle \lesseqgtr 0 .
$$

Die Facetten maximaler Dimension sind dabei offene Teilmengen von $V$ und heißen Zimmer. 
Beispielhaft sei das zweidimensionale Wurzelsystem vom Typ $\mathrm{A}_{2}$ mit seinem Kowurzelsystem gezeichnet. Die gestrichelten Geraden stellen die $H(\alpha)$ dar, auf deren Beschriftung der Übersichtlichkeit halber verzichtet wurde.
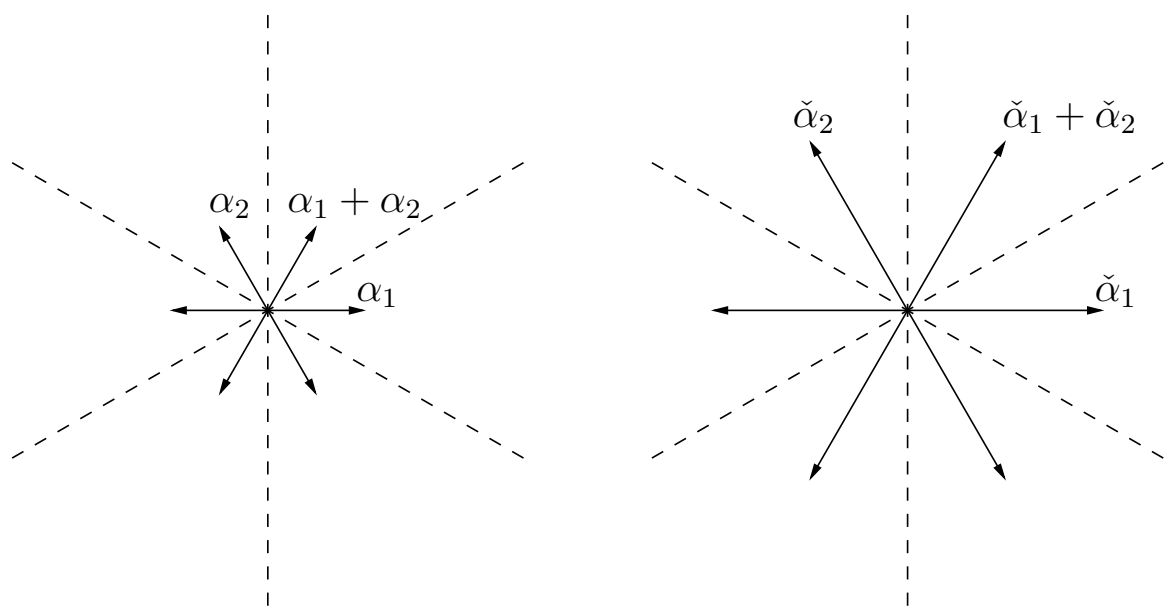

Die Skalierung der Längen der Vektoren ist dabei für einen der Räume beliebig; hier wurden die Wurzeln so gewählt, dass sie die euklidische Länge 1 darstellen sollen, daher mussten die Kowurzeln entsprechend doppelt so lang gewählt werden.

Die strikte Trennung von Vektor- und Dualraum macht es möglich, die Basiswurzeln $\alpha_{1}$ und $\alpha_{2}$ orthogonal bezüglich des standard-euklidischen Skalarprodukts zu wählen. Dabei wird deutlich, dass der Dualraum wirklich etwas anderes ist, denn die Kowurzeln bieten dann ein erkennbar anderes Bild als die Wurzeln:
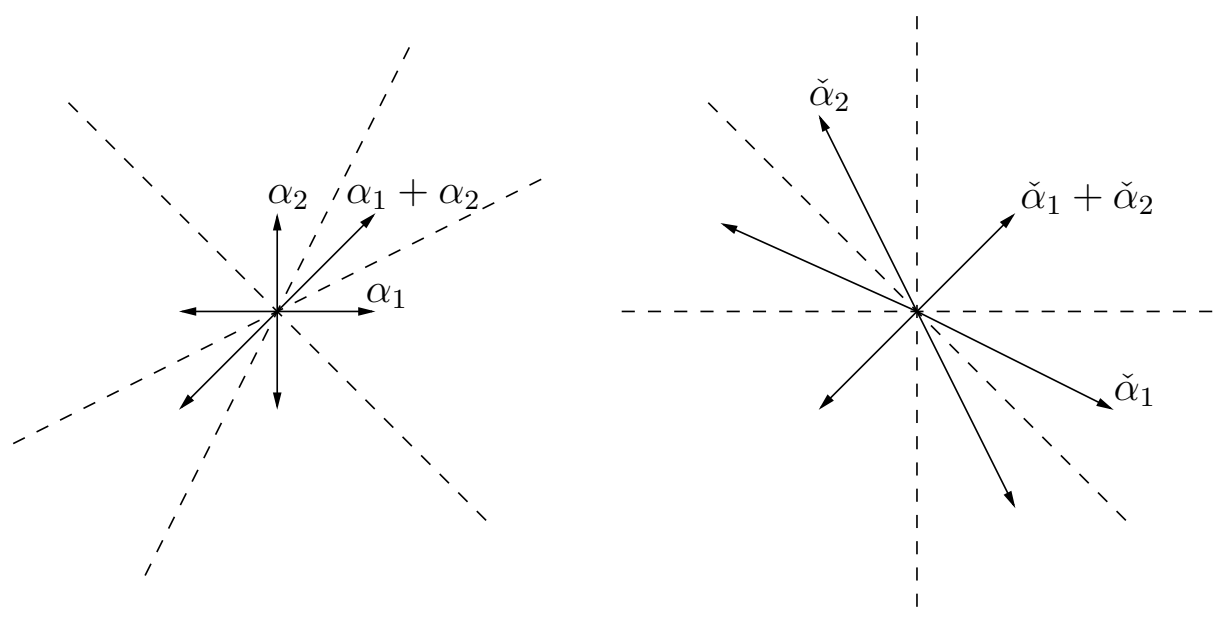

Allerdings ist hierbei zu bemerken, dass jeder der beiden Räume ein inneres Skalarprodukt besitzt, das invariant unter der Weylgruppe ist. Die sich daraus ergebende Metrik wird in dieser Darstellung missachtet. 
Für den in dieser Arbeit besonders interessierenden Fall des Wurzelsystems $\mathrm{G}_{2}$ sehen die Abbildungen folgendermaßen aus:
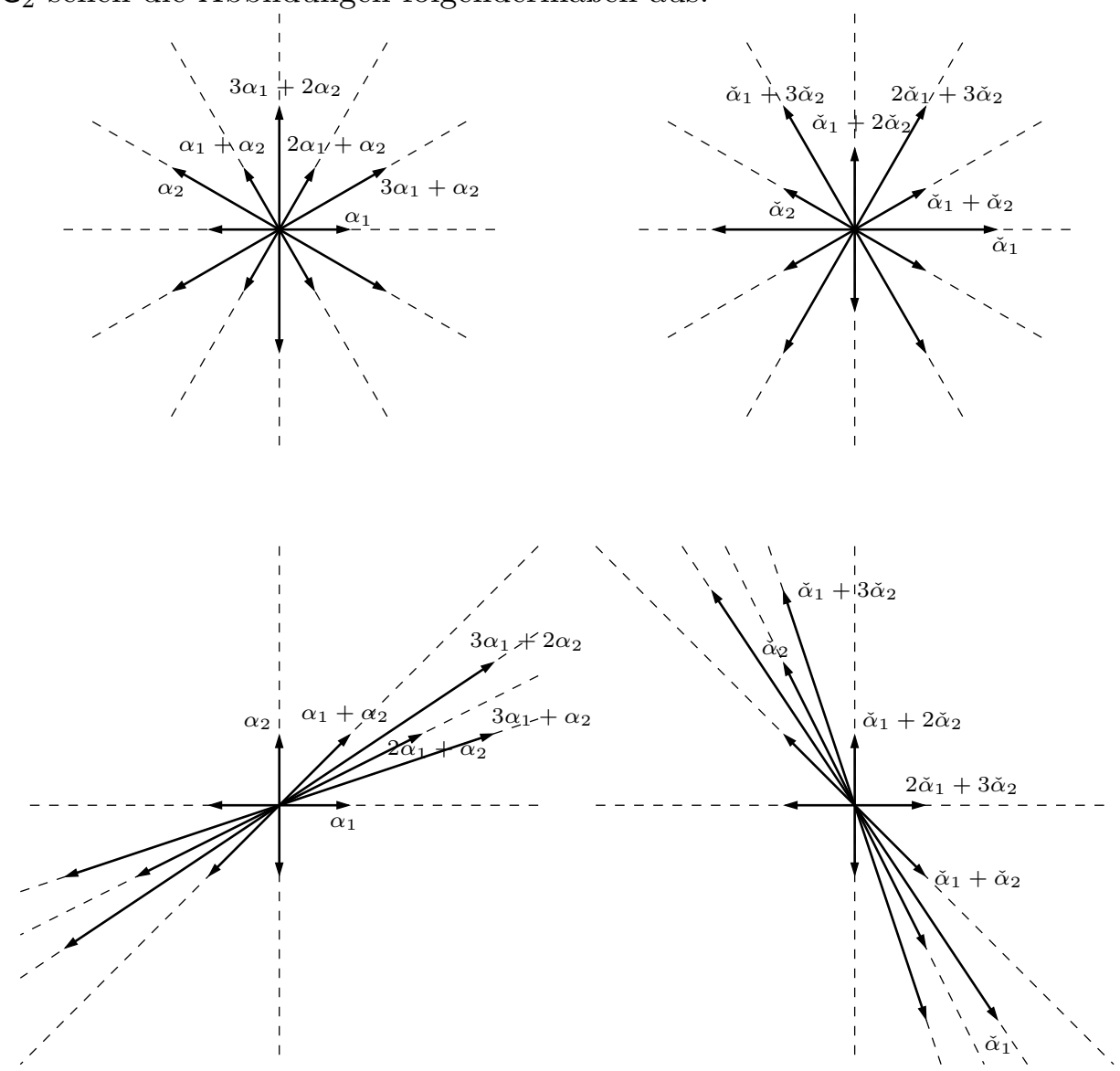

Man beachte, dass die Kowurzelbildung nicht mit der Addition verträglich ist und zum Beispiel für $G_{2}$ die Beziehungen $\left(\alpha_{1}+\alpha_{2}\right)^{\vee}=\check{\alpha}_{1}+3 \check{\alpha}_{2}$, $\left(2 \alpha_{1}+\alpha_{2}\right)^{\vee}=2 \check{\alpha}_{1}+3 \check{\alpha}_{2},\left(3 \alpha_{1}+\alpha_{2}\right)^{\vee}=\check{\alpha}_{1}+\check{\alpha}_{2}$ und $\left(3 \alpha_{1}+2 \alpha_{2}\right)^{\vee}=\check{\alpha}_{1}+2 \check{\alpha}_{2}$ gelten.

Auf der Menge der Facetten kann man über $F_{1} \leq F_{2}: \Leftrightarrow F_{1} \subseteq \bar{F}_{2}$ (diese Definition ist nach [HS03 zitiert, sie ist anders als in Beh95]) eine Partialordnung definieren. Mit $\mathcal{F}(V, \Phi)$ werde die entsprechend teilgeordnete Menge der Facetten bezeichnet, und $\mathcal{C}=\mathcal{C}(V, \Phi)$ bezeichne die Menge der Zimmer.

Die Wurzeln sind Teilmenge des Gitters der Gewichte

$$
\Lambda:=\left\{\lambda \in V \mid\langle\lambda, \check{\alpha}\rangle \in \mathbb{Z} \text { für alle } \check{\alpha} \in \Phi^{\vee}\right\} ;
$$

ebenso gehören die Kowurzeln zum Kogewichtegitter

$$
\Lambda^{\vee}:=\left\{\check{\lambda} \in V^{*} \mid\langle\alpha, \check{\lambda}\rangle \in \mathbb{Z} \text { für alle } \alpha \in \Phi\right\} .
$$


Eine Wurzelbasis oder Basis $\left\{\alpha_{1}, \ldots, \alpha_{n}\right\}=\Delta$ eines Wurzelsystems $\Phi$ ist eine Basis des Gewichtegitters mit der Eigenschaft, dass es eine disjunkte Zerlegung

$$
\Phi=\Phi_{+} \cup \Phi_{-}
$$

in die Menge

$$
\Phi_{+}:=\left\{\alpha \in \Phi \mid \alpha=\sum_{i=1}^{n} r_{i} \alpha_{i}, r_{i} \geq 0 \text { für alle } i\right\}
$$

der positiven Wurzeln und diejenige der negativen Wurzeln

$$
\Phi_{-}:=\left\{\alpha \in \Phi \mid \alpha=\sum_{i=1}^{n} r_{i} \alpha_{i}, r_{i} \leq 0 \text { für alle } i\right\}
$$

gibt.

Auch hierzu gibt es selbstverständlich die dualen Begriffe $\Phi_{+}^{\vee}$ und $\Phi_{-}^{\vee}$ zu $\Delta^{\vee}=\left\{\check{\alpha}_{1}, \ldots, \check{\alpha}_{n}\right\}$.

Zu jedem Zimmer $C$ gibt es eine Wurzelbasis $\left\{\alpha_{1}, \ldots, \alpha_{n}\right\}$ so, dass $C$ im positiven Halbraum bezüglich jedes Basiselements liegt:

$$
\forall x \in C, \forall i:\left\langle x, \alpha_{i}\right\rangle>0 .
$$

Dadurch ist eine Bijektion zwischen den Zimmern und den Wurzelbasen gegeben.

Mit Bezug auf eine gegebene Wurzelbasis $\left\{\alpha_{1}, \ldots, \alpha_{n}\right\}$ heißt ein Gewicht $\lambda \in \Lambda$ dominant, wenn $\left\langle\lambda, \check{\alpha}_{i}\right\rangle \geq 0$ für alle $i$ gilt.

Eine Menge

$$
\left\{\lambda_{1}, \ldots, \lambda_{n}\right\} \subset \Lambda
$$

von Gewichten wird als System fundamental dominanter Gewichte zu einer gegebenen Wurzelbasis $\left\{\alpha_{1}, \ldots, \alpha_{n}\right\}$ bezeichnet, wenn $\left\langle\lambda_{i}, \check{\alpha}_{j}\right\rangle=\delta_{i j}$ gilt.

Mit der im vorhergenden Absatz bezeichneten Bijektion kann man ein System fundamental dominanter Gewichte auch eineindeutig einem bestimmten Zimmer zuordnen.

Schließlich heißt ein Gewicht $\lambda \in \Lambda$ fundamental, wenn es eine Wurzelbasis gibt, bezüglich der es fundamental dominant ist.

$\mathrm{Zu}$ einem fundamentalen Gewicht $\lambda$ gibt es auf natürliche Weise ein duales fundamentales Gewicht $\check{\lambda}$ : Wenn für ein $i$ und eine beliebige Wurzelbasis $\left\{\alpha_{1}, \ldots, \alpha_{n}\right\}$ die Beziehung $\left\langle\lambda, \check{\alpha}_{j}\right\rangle=\delta_{i j}$ gilt, dann ist $\check{\lambda}$ durch $\left\langle\alpha_{j}, \check{\lambda}\right\rangle=\delta_{j i}$ gegeben.

Die zu einer gegebenen Facette $F$ duale Facette $F^{\vee}$ ergibt sich analog über die Zuordnung

$$
\forall \alpha \in \Phi: \quad\left(\langle F, \check{\alpha}\rangle \lesseqgtr 0 \quad \Longleftrightarrow \quad\left\langle\alpha, F^{\vee}\right\rangle \lesseqgtr 0\right) .
$$


Zur Ergänzung der Beschreibung seien die fundamentalen Gewichte zum Wurzelsystem $A_{2}$ angegeben:

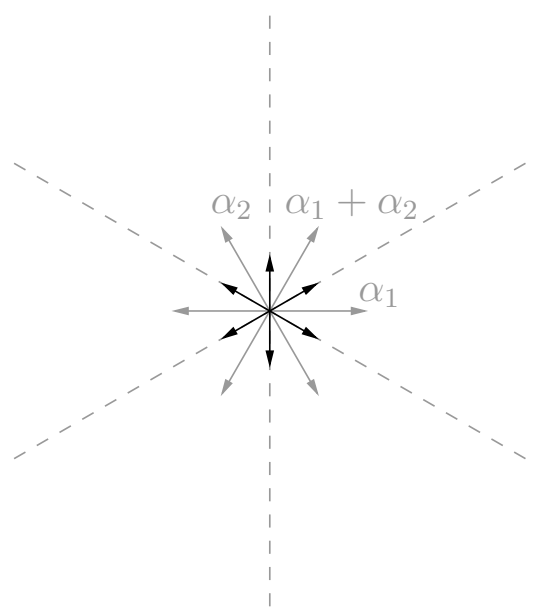

Die fundamentalen Gewichte zum Wurzelsystem $G_{2}$ entsprechen hingegen genau den Wurzeln, daher erübrigt sich eine weitere Abbildung.

\subsection{Ecken von Facetten}

Definition und Bemerkung 5.3 Sei F eine Facette von $(V, \Phi)$. Ein fundamental dominantes Gewicht $\lambda \in \Lambda$ heißt genau dann eine Ecke der Facette $F$, wenn es im Abschluss $\bar{F}$ der Facette in $V$ enthalten ist. Die Menge aller Ecken von $F$ sei mit vert $(F)$ bezeichnet.

Sie sind auch in der Menge

$$
\operatorname{vert}^{\prime}(F):=\{\lambda \in \Lambda \mid \exists C \in \mathcal{C}: \bar{C} \supset F, \lambda \in \operatorname{vert}(C)\},
$$

enthalten. Für Facetten $F_{1} \leq F_{2}$ gilt:

$$
\begin{aligned}
\operatorname{vert}\left(F_{1}\right) & \subseteq \operatorname{vert}\left(F_{2}\right) \text { und } \\
\operatorname{vert}^{\prime}\left(F_{1}\right) & \supseteq \operatorname{vert}^{\prime}\left(F_{2}\right) .
\end{aligned}
$$

Bemerkung 5.4 Geometrisch gesehen ist ein Zimmer ein von Hyperebenen begrenzter offener Kegel bzw. sein Abschluss ist ein simplizialer Kegel. Als „Ecken" eines solchen Zimmers kann man die Schnittstrahlen von je $n-1$ Hyperebenen des Kegelrands interpretieren. Aus der Definition eines fundamental dominanten Gewichts heraus ist zu erkennen, dass es zu jeder solchen "Ecke" eines Zimmers genau eine Ecke im Sinne der obigen Definition gibt und dass es keine weiteren Ecken in ihrem Sinne geben kann; der Eckenvektor erzeugt den Schnittstrahl.

$D a$ der Abschluss eines Zimmers ein simplizialer Kegel ist, ist zudem klar, dass für jedes $\lambda \in \operatorname{vert}(F)$ die Menge $\operatorname{vert}(F) \backslash\{\lambda\}$ ebenfalls die Eckenmenge einer Facette ist. 
Umgekehrt besteht $\operatorname{vert}^{\prime}(F)$ genau aus den $\lambda \in \Lambda$, für die es eine Facette $F^{\prime}$ mit $\operatorname{vert}\left(F^{\prime}\right)=\operatorname{vert}(F) \cup\{\lambda\}$ gibt.

\section{Lemma 5.5}

1. Sei $F$ eine Facette. Dann ist vert $(F)$ eine endliche Menge linear unabhängiger Vektoren.

2. Sei $\operatorname{vert}(F)=\left\{\lambda_{1}, \ldots, \lambda_{k}\right\}$.

Dann ist $F=\left\{x \in V \mid \exists a_{1}, \ldots, a_{k}>0: x=\sum_{i=1}^{k} a_{i} \lambda_{i}\right\}$.

\subsection{Reduktion und Projektion von Wurzelsystemen}

Lemma 5.6 Sei $F \in \mathcal{F}(V, \Phi)$ eine Facette. Dann erhält man folgende Zerlegungen in direkte Summen:

$$
\begin{aligned}
V & =\langle F\rangle \oplus\left(F^{\vee}\right)^{\perp} \\
V^{*} & =\left\langle F^{\vee}\right\rangle \oplus F^{\perp} .
\end{aligned}
$$

Die spitzen Klammern stehen hier für den aufgespannten Vektorraum und ()$^{\perp}$ für das orthogonale Komplement im Dualraum.

Die strikte Trennung in Raum und Dualraum lässt hier einen Beweis geboten erscheinen:

$\left\langle F^{\vee}\right\rangle$ ist per Definition das orthogonale Komplement zu der Menge der Wurzeln, deren Kowurzeln zu $F$ orthogonal sind: $\forall \alpha \in \Phi:(\langle F, \check{\alpha}\rangle=0 \Leftrightarrow$ $\left.\left\langle\alpha, F^{\vee}\right\rangle=0\right)$. Daher wird $\left\langle F^{\vee}\right\rangle^{\perp}$ genau von $O R(F):=\{\alpha \in \Phi \mid\langle F, \check{\alpha}\rangle=0\}$ aufgespannt. Der Schnitt von $\langle F\rangle$ und $\left(F^{\vee}\right)^{\perp}=\left\langle F^{\vee}\right\rangle^{\perp}$ ist somit genau dann trivial, wenn kein Element von $O R(F)$ zu $O R^{\vee}(F):=\left\{\check{\alpha} \in \Phi^{\vee} \mid \alpha \in\right.$ $O R(F)\}$ orthogonal ist. Das wiederum ist genau dann der Fall, wenn die bilineare Paarung von $O R(F)$ und $O R^{\vee}(F)$ nicht ausartet. Insbesondere gilt dies also für jedes endliche Wurzelsystem, worauf sich die Betrachtungen hier bekanntlich beschränken.

Es sei $V_{F}:=\left(F^{\vee}\right)^{\perp}, V_{F}^{*}:=F^{\perp}$ definiert. Damit ist $\Phi_{F}:=\Phi \cap V_{F}$ ein Wurzelsystem in $V_{F}$, genannt die Reduktion des Wurzelsystems $\Phi$ auf die Facette $F$. Man kann $\Phi_{F}^{\vee}:=\Phi^{\vee} \cap V_{F}^{*}$ als das duale Wurzelsystem bezüglich der Identifikation des Dualraums von $V_{F}$ mit $V_{F}^{*}$ auffassen.

Lemma 5.7 Die Projektion

$$
\text { pr: } V \rightarrow V_{F},
$$

die sich aus der direkten Summenzerlegung $V=\langle F\rangle \oplus\left(F^{\vee}\right)^{\perp}=\langle F\rangle \oplus V_{F}$ ergibt, induziert eine Bijektion

$$
\begin{aligned}
\operatorname{pr}:\left\{F^{\prime} \in \mathcal{F}(V, \Phi): F^{\prime} \geq F\right\} & \rightarrow \mathcal{F}\left(V_{F}, \Phi_{F}\right), \\
F^{\prime} & \mapsto \operatorname{pr}\left(F^{\prime}\right) .
\end{aligned}
$$


Dabei gilt: $\operatorname{dim} \operatorname{pr}\left(F^{\prime}\right)=\operatorname{dim}\left(F^{\prime}\right)-\operatorname{dim}(F)$. Insbesondere erhält man eine Bijektion der Mengen der Zimmer, die ebenso mit pr bezeichnet wird:

$$
\text { pr: }\{C \in \mathcal{C}(V, \Phi): C \geq F\} \rightarrow \mathcal{C}\left(V_{F}, \Phi_{F}\right) .
$$

Mit $\Lambda_{F}$ sei die Menge der fundamentalen Gewichte in $V_{F}$ bezeichnet.

Lemma 5.8 Die Projektion pr induziert eine Bijektion

$$
\begin{aligned}
\mathrm{pr}: \quad \operatorname{vert}^{\prime}(F)-\operatorname{vert}(F) & \rightarrow \Lambda_{F} \\
\lambda & \mapsto \operatorname{pr}(\lambda) .
\end{aligned}
$$

\subsection{Komplementärpolyeder}

Definition 5.9 Sei $(V, \Phi)$ ein Wurzelsystem. Ein Komplementärpolyeder zu $(V, \Phi)$ ist eine Abbildung $d: \mathcal{C}(V, \Phi) \rightarrow V^{*}, C \mapsto d(C)$, die die folgenden beiden Bedingungen erfüllt:

1. Seien $C, D \in \mathcal{C}(V, \Phi)$ Zimmer und $\lambda \in \operatorname{vert}(C) \cap \operatorname{vert}(D)$ eine gemeinsame Ecke, dann gilt

$$
\langle\lambda, d(C)\rangle=\langle\lambda, d(D)\rangle .
$$

2. Für je zwei benachbarte Zimmer $C, D \in \mathcal{C}(V, \Phi)$ (das heißt, dass der Schnitt ihrer jeweiligen Abschlüsse Kodimension 1 hat) und eine Wurzel $\alpha \in \Phi$ mit den Eigenschaften $\left.\check{\alpha}\right|_{C}>0$ und $\left.\check{\alpha}\right|_{D}<0$ gilt:

$$
\langle\alpha, d(C)\rangle \leq\langle\alpha, d(D)\rangle .
$$

Definition 5.10 Das duale Polyeder $d(F)$ zu einer Facette $F \in \mathcal{F}(V, \Phi)$ in Bezug auf ein gegebenes Komplementärpolyeder $d: \mathcal{C} \rightarrow V^{*}$ ist definiert als

$$
d(F):=\text { konvexe Hülle }\{d(C) \mid \bar{C} \supseteq F\} .
$$

Insbesondere ist also

$$
d(\{0\})=\text { konvexe Hülle }\{d(C) \mid C \in \mathcal{C}(V, \Phi)\}
$$

das vollständige Polyeder im geometrischen Sinne.

Bemerkung 5.11 In geometrischer Umschreibung bedeuten die beiden Forderungen an ein Komplementärpolyeder:

1. Die Bildpunkte aller Zimmer mit einer gemeinsamen Ecke $\lambda$ liegen in einer Ebene, die orthogonal zu $\lambda$ ist.

Dies rechtfertigt, speziell für das duale Polyeder, die Benutzung des Begriffs Polyeder.

2. Die zweite Bedingung fixiert die Orientierung des Komplementärpolyeders bzw. des dualen Polyeders. Quasi durch eine Punktspiegelung wird die Orientierung im Vergleich zur Anordnung der Ecken in $V$ umgedreht (wenn man gedanklich $V$ und $V^{*}$ übereinanderlegt und eine Darstellung gewählt hat, in der die Wurzeln und ihre Kowurzeln dann in dieselbe Richtung weisen). 
Beispielhaft sei ein Komplementärpolyeder zum Wurzelsystem $A_{2}$ gezeigt: Die Zimmer zum Wurzelsystem sind mit $A$ bis $F$ bezeichnet, im rechten Teil ist ein Komplementärpolyeder von in gewisser Weise kanonischer Gestalt (wegen der gleichen Längen seiner Seiten) zusammen mit seiner konvexen Hülle angegeben. Gestrichelt ist eine weitere Möglichkeit für eine konvexe Hülle eines Komplementärpolyeders gezeigt. Die Figuren können jeweils beliebig in $V^{*}$ verschoben werden.

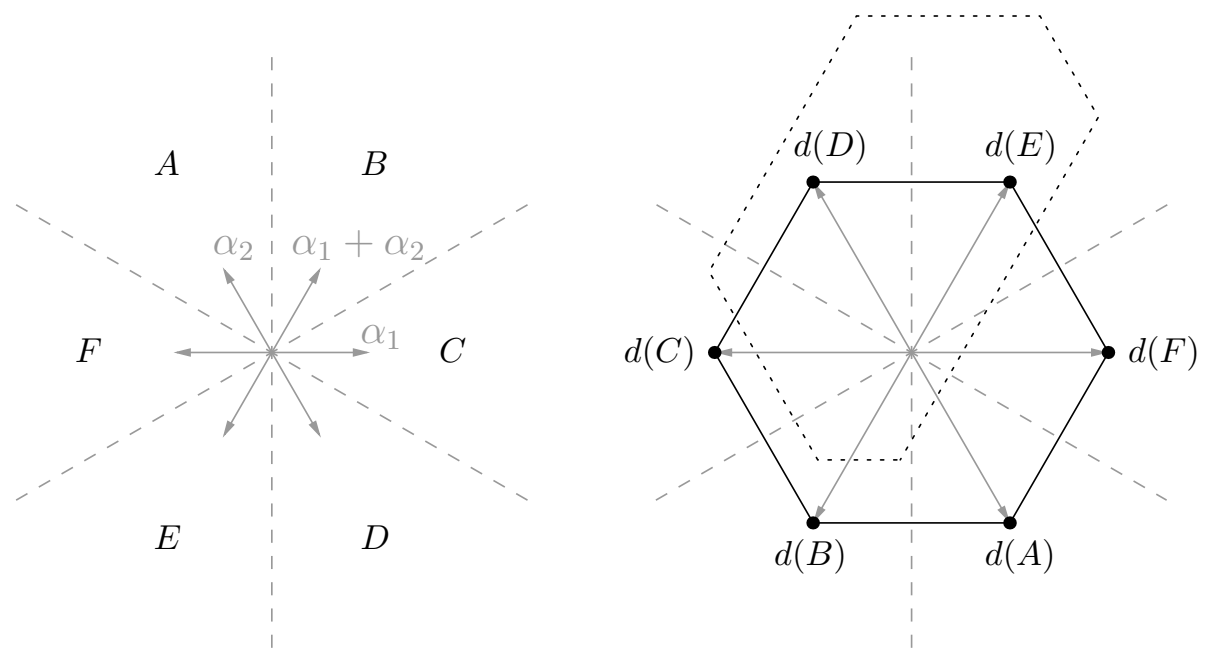

\subsection{Stabilität und spezielle Facetten}

Definition 5.12 Sei $(V, \Phi)$ ein Wurzelsystem und $F \in \mathcal{F}(V, \Phi)$ eine Facette. Es sei definiert:

$$
\begin{aligned}
& R(F):=\{\alpha \in \Phi \mid\langle\alpha, \check{\lambda}\rangle \geq 0 \text { für alle } \lambda \in \operatorname{vert}(F)\} \\
& U(F):=\{\alpha \in R(F) \mid \exists \lambda \in \operatorname{vert}(F):\langle\alpha, \check{\lambda}\rangle>0\} .
\end{aligned}
$$

Sei ferner $d: \mathcal{C} \rightarrow V^{*}$ ein Komplementärpolyeder. Dann wird der Grad von $F$ definiert als

$$
\begin{aligned}
\operatorname{deg}(F) & :=\sum_{\alpha \in U(F)}\langle\alpha, d(C)\rangle \\
& =\sum_{\alpha \in R(F)}\langle\alpha, d(C)\rangle,
\end{aligned}
$$

wobei $C \in \mathcal{C}(V, \Phi)$ beliebig unter der Voraussetzung $\bar{C} \supseteq F$ gewählt werden kann.

Beispiel: Für das Wurzelsystem $\mathrm{A}_{2}$ mit dem oben genannten Komplementärpolyeder in kanonischer Gestalt hat jedes Zimmer den Grad -4 und jede Ecke den Grad -3. 
Lemma 5.13 Die folgenden Eigenschaften sind zueinander äquivalent:

1. Für jede Facette $F \in \mathcal{F}(V, \Phi)$ gilt $\operatorname{deg}(F) \leq 0$.

2. Für jede Facette $F \in \mathcal{F}(V, \Phi)$ mit $\operatorname{dim}(F)=1$ gilt $\operatorname{deg}(F) \leq 0$.

3. Es ist $0 \in d(F)$.

Zum Beweis siehe [HS03, Lemma 3.2].

Definition 5.14 Ein Komplementärpolyeder, das die (äquivalenten) Bedingungen aus Lemma 5.13 erfüllt, heißt semistabil.

Der Begriff der Stabilität von Komplementärpolyedern wird in [Beh95] und [HS03] genutzt, um Stabilitätseigenschaften auf reduktiven Gruppen zu untersuchen. In der vorliegenden Arbeit wird der weitere Schwerpunkt auf die konkrete Betrachtung der Komplementärpolyeder gelegt. Natürlich auch in der Hoffnung, dass daraus später bessere Erkenntnisse über Stabilitätseigenschaften gewonnen werden können. 


\section{Die Automorphismengruppe der Oktaven}

\subsection{Die Weyl-Gruppe}

In diesem Kapitel soll die Struktur der Automorphismengruppe als eine reduktive Gruppe im Konkreten untersucht werden. $\mathrm{Zu}$ den Grundbegriffen für reduktive Gruppen siehe beispielweise bei [Bor91] oder [Ker07.

Als Oktaven werden hier weiterhin die zerfallenden Oktaven behandelt, und zwar in der Darstellung $C=D \oplus D \boldsymbol{a}$, wie sie sich aus dem in Abschnitt 2.3 vorgestellten Verdopplungsverfahren ergibt. Dabei ist $D$ die Algebra der reellen $(2 \times 2)$-Matrizen, $\boldsymbol{a}$ wurde in Definition 2.15 eingeführt. Als Parameter im Verdopplungsverfahren wurde $\lambda=1$ gewählt.

Mit $e_{i j}$ werde die $(2 \times 2)$-Matrix mit genau einem Eintrag ungleich null, und zwar einer Eins in der Zeile $i$ in der $j$-ten Spalte, bezeichnet. Die Automorphismengruppe der Oktaven werde $G$ genannt.

Als Erstes wird ein Torus als Standard-Torus in $G$ ausgewählt. Springer und Veldkamp beschreiben eine mögliche Wahl in [SV00, Lemma 2.3.1] als die Gruppe $T=\left\{t_{\kappa, \mu} \mid \kappa, \mu \in \mathbb{R}^{*}\right\}$ aller Automorphismen

$$
t_{\kappa, \mu}: x+y \boldsymbol{a} \mapsto\left(\begin{array}{cc}
\kappa & 0 \\
0 & \kappa^{-1}
\end{array}\right) x\left(\begin{array}{cc}
\kappa^{-1} & 0 \\
0 & \kappa
\end{array}\right)+\left(\begin{array}{cc}
\mu & 0 \\
0 & \mu^{-1}
\end{array}\right) y\left(\begin{array}{cc}
\kappa^{-1} & 0 \\
0 & \kappa
\end{array}\right) \boldsymbol{a} .
$$

Die Gewichte, also die Charaktere $T \rightarrow \mathbb{R}$ auf dem Torus, sowie die zugehörigen Gewichtsräume zu $T$ sind:

\begin{tabular}{l|c|c|c|c|c|c|c} 
Gewicht & 1 & $\kappa^{2}$ & $\kappa^{-2}$ & $\mu \kappa^{-1}$ & $\mu^{-1} \kappa$ & $\mu \kappa$ & $\mu^{-1} \kappa^{-1}$ \\
\hline $\begin{array}{l}\text { Gewichtsraum } \\
\text { aufgespannt von }\end{array}$ & $e_{11}, e_{22}$ & $e_{12}$ & $e_{21}$ & $e_{11} \boldsymbol{a}$ & $e_{22} \boldsymbol{a}$ & $e_{12} \boldsymbol{a}$ & $e_{21} \boldsymbol{a}$
\end{tabular}

Man kann die Automorphismen offensichtlicherweise auch als eine Untergruppe der $\mathrm{GL}_{8}$ auffassen. Häufig ist das Rechnen in dieser Gruppe einfacher nachzuvollziehen.

Für die Matrixdarstellung wird dann hier die folgende Reihenfolge der Basiselemente gewählt:

$$
\left\{e_{11}, e_{22}, e_{12}, e_{21}, e_{11} \boldsymbol{a}, e_{22} \boldsymbol{a}, e_{12} \boldsymbol{a}, e_{21} \boldsymbol{a}\right\} .
$$

Als ein Element der GL 8 lässt sich $t_{\kappa, \mu}$ schreiben als

$$
t_{\kappa, \mu}=\operatorname{diag}\left(1,1, \kappa^{2}, \kappa^{-2}, \mu \kappa^{-1}, \mu^{-1} \kappa, \mu \kappa, \mu^{-1} \kappa^{-1}\right) .
$$

Zur Erinnerung - vergleiche Satz 2.8, wobei ja $\lambda=1$ gewählt wurde -: Die Multiplikationsvorschrift in $D$ lautet

$$
(x+y \boldsymbol{a})(u+v \boldsymbol{a})=x u+\bar{v} y+(v x+y \bar{u}) \boldsymbol{a},
$$

und die Norm ist

$$
N(x+y \boldsymbol{a})=\operatorname{det}(x)-\operatorname{det}(y) .
$$


Aus der Multiplikationsvorschrift - alternativ kann man die Darstellung aus Bemerkung 2.14 nutzen - ergibt sich die folgende Multiplikationstabelle für die Standardbasis (mit $L=$ linker bzw. $R=$ rechter Faktor):

\begin{tabular}{l||l|l|l|l|l|l|l|l|}
\multicolumn{1}{c||}{$R$} & $e_{11}$ & $e_{22}$ & $e_{12}$ & $e_{21}$ & $e_{11} \boldsymbol{e}$ & $e_{22} \boldsymbol{a}$ & $e_{12} \boldsymbol{a}$ & $e_{21} \boldsymbol{a}$ \\
\hline$e_{11}$ & $e_{11}$ & 0 & $e_{12}$ & 0 & $e_{11} \boldsymbol{a}$ & 0 & 0 & $e_{21} \boldsymbol{a}$ \\
\hline$e_{22}$ & 0 & $e_{22}$ & 0 & $e_{21}$ & 0 & $e_{22} \boldsymbol{a}$ & $e_{12} \boldsymbol{a}$ & 0 \\
\hline$e_{12}$ & 0 & $e_{12}$ & 0 & $e_{11}$ & $e_{12} \boldsymbol{a}$ & 0 & 0 & $e_{22} \boldsymbol{a}$ \\
\hline$e_{21}$ & $e_{21}$ & 0 & $e_{22}$ & 0 & 0 & $e_{21} \boldsymbol{a}$ & $e_{11} \boldsymbol{a}$ & 0 \\
\hline$e_{11} \boldsymbol{a}$ & 0 & $e_{11} \boldsymbol{a}$ & $-e_{12} \boldsymbol{a}$ & 0 & 0 & $e_{11}$ & 0 & $-e_{21}$ \\
\hline$e_{22} \boldsymbol{a}$ & $e_{22} \boldsymbol{a}$ & 0 & 0 & $-e_{21} \boldsymbol{a}$ & $e_{22}$ & 0 & $-e_{12}$ & 0 \\
\hline$e_{12} \boldsymbol{a}$ & $e_{12} \boldsymbol{a}$ & 0 & 0 & $-e_{11} \boldsymbol{a}$ & 0 & $e_{12}$ & 0 & $-e_{22}$ \\
\hline$e_{21} \boldsymbol{a}$ & 0 & $e_{21} \boldsymbol{a}$ & $-e_{22} \boldsymbol{a}$ & 0 & $e_{21}$ & 0 & $-e_{11}$ & 0 \\
\hline
\end{tabular}

Zum Beispiel mit Hilfe der Multiplikationstabelle liefert eine systematische Untersuchung (siehe unten) diejenigen Automorphismen der Oktaven, die die vom Torus gegebene Gewichtsraumstruktur respektieren, sprich: im Normalisator des Torus liegen. Damit erhält man - nach Herausteilen des Zentralisators, der hier gleich dem Torus selbst ist - die in der folgenden Tabelle angegebenen Repräsentanten. Sie bilden zusammen die Weylgruppe zum Torus $T$.

\begin{tabular}{l||c|c|c|c|c|c|c|c|} 
& $e_{11}$ & $e_{22}$ & $e_{12}$ & $e_{21}$ & $e_{11} \boldsymbol{a}$ & $e_{22} \boldsymbol{a}$ & $e_{12} \boldsymbol{a}$ & $e_{21} \boldsymbol{a}$ \\
\hline \hline$\tau$ (Def.) & $e_{11}$ & $e_{22}$ & $e_{12}$ & $e_{21}$ & $e_{21} \boldsymbol{a}$ & $-e_{12} \boldsymbol{a}$ & $e_{22} \boldsymbol{a}$ & $-e_{11} \boldsymbol{a}$ \\
\hline$\sigma$ (Def.) & $e_{22}$ & $e_{11}$ & $e_{22} \boldsymbol{a}$ & $e_{11} \boldsymbol{a}$ & $e_{12} \boldsymbol{a}$ & $-e_{21} \boldsymbol{a}$ & $-e_{12}$ & $e_{21}$ \\
\hline$\sigma^{2}$ & $e_{11}$ & $e_{22}$ & $-e_{21} \boldsymbol{a}$ & $e_{12} \boldsymbol{a}$ & $-e_{12}$ & $-e_{21}$ & $-e_{22} \boldsymbol{a}$ & $e_{11} \boldsymbol{a}$ \\
\hline$\sigma^{3}$ & $e_{22}$ & $e_{11}$ & $-e_{21}$ & $-e_{12}$ & $-e_{22} \boldsymbol{a}$ & $-e_{11} \boldsymbol{a}$ & $e_{21} \boldsymbol{a}$ & $e_{12} \boldsymbol{a}$ \\
\hline$\sigma^{4}$ & $e_{11}$ & $e_{22}$ & $-e_{11} \boldsymbol{a}$ & $-e_{22} \boldsymbol{a}$ & $e_{21} \boldsymbol{a}$ & $-e_{12} \boldsymbol{a}$ & $e_{21}$ & $-e_{12}$ \\
\hline$\sigma^{5}$ & $e_{22}$ & $e_{11}$ & $-e_{12} \boldsymbol{a}$ & $e_{21} \boldsymbol{a}$ & $e_{21}$ & $e_{12}$ & $e_{11} \boldsymbol{a}$ & $-e_{22} \boldsymbol{a}$ \\
\hline$\tau \sigma$ & $e_{22}$ & $e_{11}$ & $-e_{12} \boldsymbol{a}$ & $e_{21} \boldsymbol{a}$ & $e_{22} \boldsymbol{a}$ & $e_{11} \boldsymbol{a}$ & $-e_{12}$ & $e_{21}$ \\
\hline$\tau \sigma^{2}$ & $e_{11}$ & $e_{22}$ & $e_{11} \boldsymbol{a}$ & $e_{22} \boldsymbol{a}$ & $-e_{12}$ & $-e_{21}$ & $e_{12} \boldsymbol{a}$ & $e_{21} \boldsymbol{a}$ \\
\hline$\tau \sigma^{3}$ & $e_{22}$ & $e_{11}$ & $-e_{21}$ & $-e_{12}$ & $e_{12} \boldsymbol{a}$ & $-e_{21} \boldsymbol{a}$ & $-e_{11} \boldsymbol{a}$ & $e_{22} \boldsymbol{a}$ \\
\hline$\tau \sigma^{4}$ & $e_{11}$ & $e_{22}$ & $-e_{21} \boldsymbol{a}$ & $e_{12} \boldsymbol{a}$ & $-e_{11} \boldsymbol{a}$ & $-e_{22} \boldsymbol{a}$ & $e_{21}$ & $-e_{12}$ \\
\hline$\tau \sigma^{5}$ & $e_{22}$ & $e_{11}$ & $-e_{22} \boldsymbol{a}$ & $-e_{11} \boldsymbol{a}$ & $e_{21}$ & $e_{12}$ & $e_{21} \boldsymbol{a}$ & $e_{12} \boldsymbol{a}$ \\
\hline
\end{tabular}

Zwar lässt sich noch $\tau$ als Abbildung

$$
\tau: x+y \boldsymbol{a} \mapsto x+\left(\begin{array}{rr}
0 & -1 \\
1 & 0
\end{array}\right) y \boldsymbol{a}
$$

darstellen, für die meisten anderen Elemente ist eine vergleichbare Darstellung jedoch nicht sinnvoll.11 Daher werden die Elemente in ihrer Wirkung in

\footnotetext{
${ }^{1} \mathrm{Da}$ sich durch Konjugation mit Elementarmatrizen aus einer gegebenen Matrix einzelne Einträge „extrahieren“ lassen, ist eine Darstellung unter Verwendung von $x$ und $y$ grundsätzlich möglich, die Terme wären jedoch viel zu unübersichtlich.
} 
Tabellenform präsentiert.

Man kann weiter nachprüfen, dass $\sigma^{6}=$ id ist und dass $\tau^{2}$ auf $D$ die Identität und auf $D \boldsymbol{a}$ die Multiplikation mit -1 ist. Da dieser Automorphismus aber in $T$ liegt, hat $\bar{\tau}$ in der Weylgruppe Ordnung 2. Somit gilt

$$
W=\left\langle\bar{\sigma}, \bar{\tau} \mid \bar{\sigma}^{6}=\mathrm{id}=\bar{\tau}^{2}, \bar{\tau} \bar{\sigma} \bar{\tau}^{-1}=\bar{\sigma}^{5}\right\rangle .
$$

Die Weylgruppe ist daher isomorph zur Diedergruppe $D_{6}$ mit 12 Elementen.

Dass die Weylgruppe der Automorphismengruppe $G$ der Oktaven isomorph zur Diedergruppe $D_{6}$ ist, lässt sich natürlich auch der Literatur entnehmen, in der festgestellt wird, dass $G$ eine reduktive Gruppe vom Typ $\mathrm{G}_{2}$ ist. (Zum Beispiel bei [SV00, Abschnitt 2.3].) Hier geht es aber auch um eine ganz konkrete Beschreibung der in diesem Zusammenhang auftretenden Objekte.

Zur Herleitung der Automorphismen $\gamma$, die den Torus respektieren: Als Idempotentes muss $e_{11}$ auf ein Idempotentes abgebildet werden, sein Bild kann daher nur $e_{11}$ oder $e_{22}$ oder $e_{11}+e_{22}$ sein. Da aber außerdem die Norm erhalten bleiben muss, bleiben nur $e_{11}$ und $e_{22}$ als mögliche Bilder. Damit steht auch das Bild von $e_{22}=e-e_{11}$ fest.

Für $e_{12}$ gilt die Gleichung $e_{11} \cdot e_{12}=e_{12}$. Daher kann im Fall $\gamma e_{11}=e_{11}$ das Bild von $e_{12}$ nur ein Vielfaches von $e_{12}, e_{11} \boldsymbol{a}$ oder $e_{21} \boldsymbol{a}$ sein. Aus der Gleichung $e_{12} \cdot e_{21}=e_{11}$ ergibt sich dann zwingend das Bild von $e_{21}$. Eine andere mögliche Argumentation wäre die, dass man beobachtet, dass die Charaktere, die als Gewichtsräume die von $e_{12}$ und $e_{21}$ aufgespannten Räume liefern, zueinander inverse Werte liefern, das muss auch für die Bilder der beiden Elementarmatrizen gelten.

Für $e_{11} \boldsymbol{a}$ und $e_{21} \boldsymbol{a}$ erkennt man aus der Struktur der Multiplikationstabelle, dass eine ganz analoge Argumentation für die beiden anderen Paare $\left\{e_{11} \boldsymbol{a}, e_{22} \boldsymbol{a}\right\}$ und $\left\{e_{12} \boldsymbol{a}, e_{21} \boldsymbol{a}\right\}$ gilt. Die noch nicht festgelegten Koeffizienten für die Bilder von $e_{12}, e_{11} \boldsymbol{a}$ und $e_{12} \boldsymbol{a}$ haben nur zwei Wahlfreiheiten, sie stehen nämlich über $e_{11} \boldsymbol{a} \cdot e_{12}=-e_{12} \boldsymbol{a}$ in Beziehung zueinander; daher gehen sie im Torus auf, wenn dieser am Ende herausgeteilt wird.

Ein möglicher solcher Automorphismus als Element der Weylgruppe lässt sich somit eindeutig beschreiben durch eine Permutation der drei Paare $\left\{e_{12}, e_{21}\right\},\left\{e_{11} \boldsymbol{a}, e_{22} \boldsymbol{a}\right\}$ und $\left\{e_{12} \boldsymbol{a}, e_{21} \boldsymbol{a}\right\}$ sowie durch die Angabe des Bildes von $e_{11}$, das gleichzeitig die genaue Verteilung der Bilder innerhalb der Paare festlegt. Damit gibt es höchstens 12 Elemente in der Weylgruppe mit der Folge, dass die Liste oben vollständig ist.

\subsection{Bestimmung der Wurzelunterguppen}

Es sollen nun die Wurzelunterguppen bestimmt werden, das heißt diejenigen einparametrigen Untergruppen, bei denen die Konjugation mit einem Toruselement der Multiplikation des Parameters mit dem Bild des Toruselements bei der Anwendung eines Charakters (Gewichtes) entspricht. 
Aus gruppentheoretischer Sicht bietet sich bei dieser Bestimmung der Zugang über die $\mathrm{GL}_{8}$ an. Wie weiter oben bereits festgestellt wurde, stellen sich Toruselemente in folgender Form (natürlich in Diagonalgestalt) dar:

$$
t_{\kappa, \mu}=\operatorname{diag}\left(1,1, \kappa^{2}, \kappa^{-2}, \kappa^{-1} \mu, \kappa \mu^{-1}, \kappa \mu, \kappa^{-1} \mu^{-1}\right) .
$$

Der Charakter $X$, für den $X\left(t_{\kappa, \mu}\right)=\kappa^{2}$ gilt, sei bei leichtem Missbrauch der Notation in Kurzform mit $X^{\kappa^{2}}$ bezeichnet, die anderen Charaktere entsprechend.

Es sei nun die Wurzelunterguppe zu $X^{\kappa^{2}}$ gesucht. Die Konjugation mit $t_{\kappa, \mu}$ ergibt, dass nur an den mit $*$ bezeichneten Stellen eines Elements (in Matrixschreibweise) der Wurzelunterguppe $U^{\kappa^{2}}$ die Einträge weder verschwinden noch 1 sein müssen; genauer müssen sie linear bzw. quadratisch (bei **) vom Parameter $\eta$ von $U^{\kappa^{2}}$ abhängen. Die Diagonaleinträge müssen konstant, also eins sein, da ja eine Untergruppe der $\mathrm{GL}_{8}$ vorliegt.

$$
\left(\begin{array}{lllccccc}
1 & 0 & 0 & * & 0 & 0 & 0 & 0 \\
0 & 1 & 0 & * & 0 & 0 & 0 & 0 \\
* & * & 1 & * * & 0 & 0 & 0 & 0 \\
0 & 0 & 0 & 1 & 0 & 0 & 0 & 0 \\
0 & 0 & 0 & 0 & 1 & 0 & 0 & 0 \\
0 & 0 & 0 & 0 & 0 & 1 & 0 & * \\
0 & 0 & 0 & 0 & * & 0 & 1 & 0 \\
0 & 0 & 0 & 0 & 0 & 0 & 0 & 1
\end{array}\right)
$$

Man rechnet relativ leicht nach, dass die Gruppe

$$
U^{\kappa^{2}}:\left\{\left(\begin{array}{rrrrrrrr}
1 & 0 & 0 & \eta & 0 & 0 & 0 & 0 \\
0 & 1 & 0 & -\eta & 0 & 0 & 0 & 0 \\
-\eta & \eta & 1 & -\eta^{2} & 0 & 0 & 0 & 0 \\
0 & 0 & 0 & 1 & 0 & 0 & 0 & 0 \\
0 & 0 & 0 & 0 & 1 & 0 & 0 & 0 \\
0 & 0 & 0 & 0 & 0 & 1 & 0 & -\eta \\
0 & 0 & 0 & 0 & -\eta & 0 & 1 & 0 \\
0 & 0 & 0 & 0 & 0 & 0 & 0 & 1
\end{array}\right) \mid \eta \in \mathbb{G}_{\mathrm{a}}\right\}
$$

kanonisch isomorph zur additiven Gruppe $\mathbb{G}_{\mathrm{a}}$ ist und zudem eine Gruppe von Automorphismen der Oktaven ist, denn sie lässt sich ebenso als Gruppe

$$
U^{\kappa^{2}}=\left\{x+y \boldsymbol{a} \mapsto\left(\begin{array}{cc}
1 & \eta \\
0 & 1
\end{array}\right) x\left(\begin{array}{rr}
1 & -\eta \\
0 & 1
\end{array}\right)+\left(\begin{array}{ll}
1 & 0 \\
0 & 1
\end{array}\right) y\left(\begin{array}{rr}
1 & -\eta \\
0 & 1
\end{array}\right) \boldsymbol{a} \mid \eta \in \mathbb{G}_{\mathrm{a}}\right\}
$$

darstellen. Mit Hilfe dieser Darstellung kann man die Automorphismeneigenschaft leicht verifizieren.

Die weiteren Wurzeluntergruppen kann man zum Beispiel sukzessive über Konjugation mit dem Weylgruppenelement $\bar{\sigma}$ ausrechnen: Es ist 


$$
\bar{\sigma} U^{\kappa^{2}} \bar{\sigma}^{-1}=\left\{\left(\begin{array}{rrrrrrrr}
1 & 0 & 0 & 0 & -\eta & 0 & 0 & 0 \\
0 & 1 & 0 & 0 & \eta & 0 & 0 & 0 \\
0 & 0 & 1 & 0 & 0 & 0 & \eta & 0 \\
0 & 0 & 0 & 1 & 0 & 0 & 0 & 0 \\
0 & 0 & 0 & 0 & 1 & 0 & 0 & 0 \\
\eta & -\eta & 0 & 0 & -\eta^{2} & 1 & 0 & 0 \\
0 & 0 & 0 & 0 & 0 & 0 & 1 & 0 \\
0 & 0 & 0 & \eta & 0 & 0 & 0 & 1
\end{array}\right) \mid \eta \in \mathbb{G}_{\mathrm{a}}\right\}=U^{\kappa \mu^{-1}}
$$

Weiter ergibt sich

$$
\left.\begin{array}{rl}
U^{(\kappa \mu)^{-1}}= & \left\{\left(\begin{array}{rrrrrrrr}
1 & 0 & 0 & 0 & 0 & 0 & \eta & 0 \\
0 & 1 & 0 & 0 & 0 & 0 & -\eta & 0 \\
0 & 0 & 1 & 0 & 0 & 0 & 0 & 0 \\
0 & 0 & 0 & 1 & \eta & 0 & 0 & 0 \\
0 & 0 & 0 & 0 & 1 & 0 & 0 & 0 \\
0 & 0 & -\eta & 0 & 0 & 1 & 0 & 0 \\
0 & 0 & 0 & 0 & 0 & 0 & 1 & 0 \\
\eta & -\eta & 0 & 0 & 0 & 0 & \eta^{2} & 1
\end{array}\right) \mid \eta \in \mathbb{G}_{\mathrm{a}}\right\} \\
U^{\kappa^{-2}} & =\left\{\left(\begin{array}{rrrrrrrrr}
1 & 0 & \eta & 0 & 0 & 0 & 0 & 0 \\
0 & 1 & -\eta & 0 & 0 & 0 & 0 & 0 \\
0 & 0 & 1 & 0 & 0 & 0 & 0 & 0 \\
-\eta & \eta & -\eta^{2} & 1 & 0 & 0 & 0 & 0 \\
0 & 0 & 0 & 0 & 1 & 0 & \eta & 0 \\
0 & 0 & 0 & 0 & 0 & 1 & 0 & 0 \\
0 & 0 & 0 & 0 & 0 & 0 & 1 & 0 \\
0 & 0 & 0 & 0 & 0 & \eta & 0 & 1
\end{array}\right) \mid \eta \in \mathbb{G}_{\mathrm{a}}\right.
\end{array}\right\}
$$




$$
U^{\kappa \mu}=\left\{\left(\begin{array}{rrrrrrrr}
1 & 0 & 0 & 0 & 0 & 0 & 0 & -\eta \\
0 & 1 & 0 & 0 & 0 & 0 & 0 & \eta \\
0 & 0 & 1 & 0 & 0 & \eta & 0 & 0 \\
0 & 0 & 0 & 1 & 0 & 0 & 0 & 0 \\
0 & 0 & 0 & -\eta & 1 & 0 & 0 & 0 \\
0 & 0 & 0 & 0 & 0 & 1 & 0 & 0 \\
-\eta & \eta & 0 & 0 & 0 & 0 & 1 & \eta^{2} \\
0 & 0 & 0 & 0 & 0 & 0 & 0 & 1
\end{array}\right) \mid \eta \in \mathbb{G}_{\mathrm{a}}\right\}
$$

Auf diese Art wurden erst sechs Wurzeluntergruppen gefunden. Nun werden die anderen sechs bestimmt, denn aus der Theorie ist bekannt, dass es genau zwölf Wurzeluntergruppen gibt.

Weitere Wurzeluntergruppen müssen zu anderen Wurzeln als den bisher gefundenen gehören. Daher hilft nun ein Blick auf die Wirkung von $T$ durch Konjugation auf $\mathrm{GL}_{8}$. Das Element

$$
t_{\kappa, \mu}=\operatorname{diag}\left(1,1, \kappa^{2}, \kappa^{-2}, \kappa^{-1} \mu, \kappa \mu^{-1}, \kappa \mu, \kappa^{-1} \mu^{-1}\right)
$$

verändert die Einträge einer $8 \times 8$-Matrix $M$ zu $t_{\kappa, \mu} M t_{\kappa, \mu}^{-1}$ durch folgende Faktoren (in offensichtlicher Notation):

$$
\left[\begin{array}{cccccccc}
1 & 1 & \kappa^{-2} & \kappa^{2} & \kappa \mu^{-1} & \kappa^{-1} \mu & \kappa^{-1} \mu^{-1} & \kappa \mu \\
1 & 1 & \kappa^{-2} & \kappa^{2} & \kappa \mu^{-1} & \kappa^{-1} \mu & \kappa^{-1} \mu^{-1} & \kappa \mu \\
\kappa^{2} & \kappa^{2} & 1 & \kappa^{4} & \kappa^{3} \mu^{-1} & \kappa \mu & \kappa \mu^{-1} & \kappa^{3} \mu \\
\kappa^{-2} & \kappa^{-2} & \kappa^{-4} & 1 & \kappa^{-1} \mu^{-1} & \kappa^{-3} \mu & \kappa^{-3} \mu^{-1} & \kappa^{-1} \mu \\
\kappa^{-1} \mu & \kappa^{-1} \mu & \kappa^{-3} \mu & \kappa \mu & 1 & \kappa^{-2} \mu^{2} & \kappa^{-2} & \mu^{2} \\
\kappa \mu^{-1} & \kappa \mu^{-1} & \kappa^{-1} \mu^{-1} & \kappa^{3} \mu^{-1} & \kappa^{2} \mu^{-2} & 1 & \mu^{-2} & \kappa^{2} \\
\kappa \mu & \kappa \mu & \kappa^{-1} \mu & \kappa^{3} \mu & \kappa^{2} & \mu^{2} & 1 & \kappa^{2} \mu^{2} \\
\kappa^{-1} \mu^{-1} & \kappa^{-1} \mu^{-1} & \kappa^{-3} \mu^{-1} & \kappa \mu^{-1} & \mu^{-2} & \kappa^{-2} & \kappa^{-2} \mu^{-2} & 1
\end{array}\right]
$$

Eine mögliche weitere Wurzel ist also beispielsweise der Charakter $X$ mit $X\left(t_{\kappa, \mu}\right)=\kappa^{3} \mu$. Eine zugehörige Wurzeluntergruppe $U^{\kappa^{3} \mu}$ darf folglich - als unipotente Gruppe, die einparametrisch mit Parameter $\eta$ dergestalt ist, dass $t_{\kappa, \mu} u_{\eta} t_{\kappa, \mu}^{-1}=u_{X\left(t_{\kappa, \mu}\right) \eta}=u_{\kappa^{3} \mu \eta}$ gilt - außer Einsen auf der Hauptdiagonalen allenfalls Einträge an zwei weiteren Stellen haben (Positionen $(3,8)$ und $(7,4)$ ), die zudem linear von $\eta$ abhängen müssen.

Sei $\omega_{\eta} \in \mathrm{GL}_{8}$ ein Element, das durch die dementsprechend gebildete Matrix

$$
\left(\begin{array}{llllllll}
1 & 0 & 0 & 0 & 0 & 0 & 0 & 0 \\
0 & 1 & 0 & 0 & 0 & 0 & 0 & 0 \\
0 & 0 & 1 & 0 & 0 & 0 & 0 & \eta \\
0 & 0 & 0 & 1 & 0 & 0 & 0 & 0 \\
0 & 0 & 0 & 0 & 1 & 0 & 0 & 0 \\
0 & 0 & 0 & 0 & 0 & 1 & 0 & 0 \\
0 & 0 & 0 & \tilde{\eta} & 0 & 0 & 1 & 0 \\
0 & 0 & 0 & 0 & 0 & 0 & 0 & 1
\end{array}\right)
$$


dargestellt wird, wobei $\tilde{\eta}$ für ein Vielfaches von $\eta$ steht. Seine Wirkung auf ein Element $x+y \boldsymbol{a} \in C$ lässt sich in Termen von $x$ und $y$ auch wie folgt beschreiben:

$$
\begin{aligned}
\omega_{\eta}(x+y \boldsymbol{a}) & =x+\eta\left(\begin{array}{cc}
0 & y_{21} \\
0 & 0
\end{array}\right)+\left(y+\tilde{\eta}\left(\begin{array}{cc}
0 & x_{21} \\
0 & 0
\end{array}\right)\right) \boldsymbol{a} \\
& =x+\eta e_{12} y e_{12}+\left(y+\tilde{\eta} e_{12} x e_{12}\right) \boldsymbol{a} .
\end{aligned}
$$

Zum Nachweis, dass auch $\omega_{\eta} \in \operatorname{Aut}(C)$ gilt, muss die Verträglichkeit mit der Multiplikation (6.1) in $C$ überprüft werden. Es ist

$$
\begin{aligned}
& \omega_{\eta}(x+y \boldsymbol{a}) \cdot \omega_{\eta}(u+v \boldsymbol{a}) \\
&=\left(x+\eta\left(\begin{array}{cc}
0 & y_{21} \\
0 & 0
\end{array}\right)+\left(y+\tilde{\eta}\left(\begin{array}{cc}
0 & x_{21} \\
0 & 0
\end{array}\right)\right) \boldsymbol{a}\right) \\
& \cdot\left(u+\eta\left(\begin{array}{cc}
0 & v_{21} \\
0 & 0
\end{array}\right)+\left(v+\tilde{\eta}\left(\begin{array}{cc}
0 & u_{21} \\
0 & 0
\end{array}\right)\right) \boldsymbol{a}\right) \\
&= x u+\eta\left(\begin{array}{ccc}
0 & x_{11} v_{21} \\
0 & x_{21} v_{21}
\end{array}\right)+\eta\left(\begin{array}{cc}
y_{21} u_{21} & y_{21} u_{22} \\
0 & 0
\end{array}\right) \\
&+\bar{v} y+\tilde{\eta}\left(\begin{array}{cc}
-y_{21} u_{21} & -y_{22} u_{21} \\
0 & 0
\end{array}\right)+\tilde{\eta}\left(\begin{array}{cc}
0 & x_{21} v_{22} \\
0 & -x_{21} v_{21}
\end{array}\right) \\
&+\left(v x+\tilde{\eta}\left(\begin{array}{cc}
x_{21} u_{21} & x_{22} u_{21} \\
0 & 0
\end{array}\right)+\eta\left(\begin{array}{cc}
0 & y_{21} v_{11} \\
0 & y_{21} v_{21}
\end{array}\right)\right. \\
&\left.+y \bar{u}+\eta\left(\begin{array}{ccc}
0 & -y_{11} v_{21} \\
0 & -y_{21} v_{21}
\end{array}\right)+\tilde{\eta}\left(\begin{array}{cc}
-x_{21} u_{21} & x_{21} u_{11} \\
0 & 0
\end{array}\right)\right) \boldsymbol{a} .
\end{aligned}
$$

Außerdem ist

$$
\begin{aligned}
& \omega_{\eta}(x u+\bar{v} y+(v x+y \bar{u}) \boldsymbol{a}) \\
& =x u+\bar{v} y+\eta\left(\begin{array}{cc}
0 & v_{21} x_{11}+v_{22} x_{21}+y_{21} u_{22}-y_{22} u_{21} \\
0 & 0
\end{array}\right) \\
& \quad+\left(\left(v x+y \bar{u}+\tilde{\eta}\left(\begin{array}{cc}
0 & x_{21} u_{11}+x_{22} u_{21}-y_{11} v_{21}+y_{21} v_{11} \\
0 & 0
\end{array}\right)\right) \boldsymbol{a} .\right.
\end{aligned}
$$

Ein Koeffizientenvergleich ergibt, dass Verträglichkeit mit der Multiplikation genau dann gegeben ist, wenn $\tilde{\eta}=\eta$ ist. In diesem Fall erkennt man auch leicht, dass genauso Verträglichkeit mit der Konjugation in $C$ erreicht ist.

Damit ist

$$
U^{\kappa^{3} \mu}=\left\{\left(\begin{array}{cccccccc}
1 & 0 & 0 & 0 & 0 & 0 & 0 & 0 \\
0 & 1 & 0 & 0 & 0 & 0 & 0 & 0 \\
0 & 0 & 1 & 0 & 0 & 0 & 0 & \eta \\
0 & 0 & 0 & 1 & 0 & 0 & 0 & 0 \\
0 & 0 & 0 & 0 & 1 & 0 & 0 & 0 \\
0 & 0 & 0 & 0 & 0 & 1 & 0 & 0 \\
0 & 0 & 0 & \eta & 0 & 0 & 1 & 0 \\
0 & 0 & 0 & 0 & 0 & 0 & 0 & 1
\end{array}\right) \mid \eta \in \mathbb{G}_{\mathrm{a}}\right\}
$$


die Wurzeluntergruppe zum Charakter $X$ mit $X\left(t_{\kappa, \mu}\right)=\kappa^{3} \mu$.

Weitere Wurzeluntergruppen kam man wieder über die Operation der WeylGruppe erhalten.

Bei solchermaßen spärlich besetzten Matrizen ist zum Nachvollziehen der Rechnungen vielleicht eine andere Notation für das Weylgruppenelement $\bar{\sigma}$ sinnvoll, dessen Repräsentant $\sigma$ als Matrix die Form

$$
\left(\begin{array}{rrrrrrrr}
0 & 1 & 0 & 0 & 0 & 0 & 0 & 0 \\
1 & 0 & 0 & 0 & 0 & 0 & 0 & 0 \\
0 & 0 & 0 & 0 & 0 & 0 & -1 & 0 \\
0 & 0 & 0 & 0 & 0 & 0 & 0 & 1 \\
0 & 0 & 0 & 1 & 0 & 0 & 0 & 0 \\
0 & 0 & 1 & 0 & 0 & 0 & 0 & 0 \\
0 & 0 & 0 & 0 & 1 & 0 & 0 & 0 \\
0 & 0 & 0 & 0 & 0 & -1 & 0 & 0
\end{array}\right)
$$

hat: es entspricht der Permutation (1 2)(4 5 7-36-8), und zwar von links auf die Zeilen bezogen und als Inverses von rechts auf die Spalten bezogen. Dabei wird an den durch ein Minuszeichen verbundenen Stellen zusätzlich das Vorzeichen gewechselt.

Auf diese Weise bekommt man fünf weitere Wurzeluntergruppen zu den Charakteren mit Werten $\kappa^{3} \mu^{-1}, \mu^{-2}, \kappa^{-3} \mu^{-1}, \kappa^{-3} \mu$ und $\mu^{2}$ :

$$
\begin{aligned}
U^{\kappa^{3} \mu^{-1}} & =\left\{\left(\begin{array}{rrrrrrrr}
1 & 0 & 0 & 0 & 0 & 0 & 0 & 0 \\
0 & 1 & 0 & 0 & 0 & 0 & 0 & 0 \\
0 & 0 & 1 & 0 & -\eta & 0 & 0 & 0 \\
0 & 0 & 0 & 1 & 0 & 0 & 0 & 0 \\
0 & 0 & 0 & 0 & 1 & 0 & 0 & 0 \\
0 & 0 & 0 & \eta & 0 & 1 & 0 & 0 \\
0 & 0 & 0 & 0 & 0 & 0 & 1 & 0 \\
0 & 0 & 0 & 0 & 0 & 0 & 0 & 1
\end{array}\right) \mid \eta \in \mathbb{G}_{\mathrm{a}}\right\} \\
U^{\mu^{-2}} & =\left\{\left(\begin{array}{rrrrrrrrr}
1 & 0 & 0 & 0 & 0 & 0 & 0 & 0 \\
0 & 1 & 0 & 0 & 0 & 0 & 0 & 0 \\
0 & 0 & 1 & 0 & 0 & 0 & 0 & 0 \\
0 & 0 & 0 & 1 & 0 & 0 & 0 & 0 \\
0 & 0 & 0 & 0 & 1 & 0 & 0 & 0 \\
0 & 0 & 0 & 0 & 0 & 1 & -\eta & 0 \\
0 & 0 & 0 & 0 & 0 & 0 & 1 & 0 \\
0 & 0 & 0 & 0 & -\eta & 0 & 0 & 1
\end{array}\right) \mid \eta \in \mathbb{G}_{\mathrm{a}}\right\}
\end{aligned}
$$




$$
\left.\begin{array}{rl}
U^{\kappa^{-3} \mu^{-1}}= & \left\{\left(\begin{array}{rrrrrrrr}
1 & 0 & 0 & 0 & 0 & 0 & 0 & 0 \\
0 & 1 & 0 & 0 & 0 & 0 & 0 & 0 \\
0 & 0 & 1 & 0 & 0 & 0 & 0 & 0 \\
0 & 0 & 0 & 1 & 0 & 0 & -\eta & 0 \\
0 & 0 & 0 & 0 & 1 & 0 & 0 & 0 \\
0 & 0 & 0 & 0 & 0 & 1 & 0 & 0 \\
0 & 0 & 0 & 0 & 0 & 0 & 1 & 0 \\
0 & 0 & -\eta & 0 & 0 & 0 & 0 & 1
\end{array}\right) \mid \eta \in \mathbb{G}_{\mathrm{a}}\right\} \\
U^{\kappa^{-3} \mu} & =\left\{\left(\begin{array}{rrrrrrrr}
1 & 0 & 0 & 0 & 0 & 0 & 0 & 0 \\
0 & 1 & 0 & 0 & 0 & 0 & 0 & 0 \\
0 & 0 & 1 & 0 & 0 & 0 & 0 & 0 \\
0 & 0 & 0 & 1 & 0 & -\eta & 0 & 0 \\
0 & 0 & \eta & 0 & 1 & 0 & 0 & 0 \\
0 & 0 & 0 & 0 & 0 & 1 & 0 & 0 \\
0 & 0 & 0 & 0 & 0 & 0 & 1 & 0 \\
0 & 0 & 0 & 0 & 0 & 0 & 0 & 1
\end{array}\right) \mid \eta \in \mathbb{G}_{\mathrm{a}}\right. \\
U^{\mu^{2}}= & \left\{\left(\begin{array}{llllllll}
1 & 0 & 0 & 0 & 0 & 0 & 0 & 0 \\
0 & 1 & 0 & 0 & 0 & 0 & 0 & 0 \\
0 & 0 & 1 & 0 & 0 & 0 & 0 & 0 \\
0 & 0 & 0 & 1 & 0 & 0 & 0 & 0 \\
0 & 0 & 0 & 0 & 1 & 0 & 0 & \eta \\
0 & 0 & 0 & 0 & 0 & 1 & 0 & 0 \\
0 & 0 & 0 & 0 & 0 & \eta & 1 & 0 \\
0 & 0 & 0 & 0 & 0 & 0 & 0 & 1
\end{array}\right) \mid \eta \in \mathbb{G}_{\mathrm{a}}\right.
\end{array}\right\}
$$

Teilweise doppelt auftretende Vorzeichen wurden beibehalten, um die Rechnungen nachvollziehbarer zu machen.

Damit sind wie angekündigt sechs weitere Wurzeluntergruppen gefunden, insgesamt also die erwarteten zwölf zu einer Gruppe vom Typ $G_{2}$. 


\section{Apartments und Normen}

Die zu einer nicht-archimedischen Primstelle gehörenden Normen auf den Oktaven stehen in enger Verbindung zum Bruhat-Tits-Gebäude über $G$. Die genaue Untersuchung dieser Verbindung ist der Gegenstand dieses Kapitels.

\subsection{Allgemeine Konstruktion eines Apartments}

Gegeben sei eine zusammenhängende, reduktive Gruppe $G$ über einem Körper $K$, der abgeschlossen bezüglich einer nichttrivialen diskreten Bewertung $\omega$ ist; der zugehörige diskrete Bewertungsring sei $\mathcal{O}$. Dann ist zu $G$ nach Tits [Tit79] (siehe auch bei Landvogt [Lan96]) das sogenannte Bruhat-TitsGebäude definiert. In diesem Gebäude gehört dabei zu jedem maximalen Torus $S$ in $G$ ein Apartment. Wesentliches Element zum Aufbau dieses Apartments ist der Raum der Ko-Charaktere des Torus:

$$
V:=X_{*}(S) \otimes_{\mathbb{Z}} \mathbb{R} .
$$

Sei $Z(K)$ die Gruppe der $K$-rationalen Elemente des Zentralisators von $S$ (jene Gruppe ist hier gleich dem Torus $S$ selbst) sowie $N(K)$ die Gruppe der $K$-rationalen Elemente des Normalisators von $S$. Es gibt einen eindeutig bestimmten Gruppenhomomorphismus

$$
\nu: Z(K) \rightarrow V
$$

derart, dass

$$
\langle\chi, \nu(z)\rangle=-\omega(\chi(z)) \text { für alle } \chi \in X_{K}^{*}(Z)
$$

ist. (Es ist dabei $\langle\cdot, \cdot\rangle$ die kanonische perfekte Paarung von $X^{*}(Z)$ mit $X_{*}(Z)$.)

Das „leere Apartment" $A$ ist der eindeutig bestimmte affine $V$-Raum mit der Eigenschaft, dass es einen eindeutigen Gruppenhomomorphismus

$$
\nu: N(K) \rightarrow \operatorname{Aff}(A)
$$

in die affinen Bijektionen von $A$ in sich gibt, der die oben genannte Abbildung $\nu: Z(K) \rightarrow V \subset \operatorname{Aff}(A)$ fortsetzt.

Ein maximaler Torus der Automorphismen der Oktaven wurde bereits bezüglich der im Abschnitt 6.1 genannten Basis

$$
\left\{e_{11}, e_{22}, e_{12}, e_{21}, e_{11} \boldsymbol{a}, e_{22} \boldsymbol{a}, e_{12} \boldsymbol{a}, e_{21} \boldsymbol{a}\right\}
$$

gegeben. Allerdings hat der Torus dort auch Einträge, die rein quadratisch sind. Da in dieser Arbeit nicht über einem algebraisch abgeschlossenen 
Körper gearbeitet wird, wird mit dieser Parametrisierung nicht der ganze Torus in einer allgemeinen Parametrisierung erreicht. Für die Bestimmung der Wurzeluntergruppen hatte dies noch keine Auswirkung; für den weiteren Verlauf ist es jedoch besser, den Torus in der Form

$$
\left\{t_{f, g}:=\operatorname{diag}\left(1,1, f, f^{-1}, g, g^{-1}, f g,(f g)^{-1}\right) \mid f, g \in K^{*}\right\}
$$

zu betrachten.

Eine Basis von $V$ besteht aus den Elementen

$$
\begin{aligned}
& a^{\vee}: f \mapsto t_{f, 1} \quad \text { und } \\
& b^{\vee}: g \mapsto t_{1, g} .
\end{aligned}
$$

Die duale Basis ist dann

$$
\begin{aligned}
& a: t_{f, g} \mapsto f, \\
& b: t_{f, g} \mapsto g .
\end{aligned}
$$

Für $\chi=k \cdot a+\ell \cdot b$ ist $\chi\left(t_{f, g}\right)=f^{k} \cdot g^{\ell}$, außerdem $\omega\left(\chi\left(t_{f, g}\right)\right)=k \cdot \omega(f)+\ell \cdot \omega(g)$. Daher muss

$$
\nu: t_{f, g} \mapsto-\omega(f) \cdot a^{\vee}-\omega(g) \cdot b^{\vee}
$$

gelten.

Weil im Weiteren nur der Grad der Parameter des Torus bezüglich der Bewertung von Interesse ist, seien zur Erleichterung der Notation die Äquivalenzklassen

$$
\hat{t}_{r, s}:=\left\{t_{f, g} \in S \mid \omega(f)=r, \omega(g)=s\right\}
$$

eingeführt. Damit kann $\nu$ wohldefiniert als

$$
\nu: \hat{t}_{r, s} \mapsto-r \cdot a^{\vee}-s \cdot b^{\vee}
$$

geschrieben werden.

Zur Bestimmung der Fortsetzung von $\nu$ auf $N(K)$ wird die Wirkung der Erzeuger $\bar{\sigma}$ und $\bar{\tau}$ der Weylgruppe auf dem Torus untersucht. Diese Elemente werden repräsentiert durch

$$
\sigma=\left(\begin{array}{rrrrrrrr}
0 & 1 & 0 & 0 & 0 & 0 & 0 & 0 \\
1 & 0 & 0 & 0 & 0 & 0 & 0 & 0 \\
0 & 0 & 0 & 0 & 0 & 0 & -1 & 0 \\
0 & 0 & 0 & 0 & 0 & 0 & 0 & 1 \\
0 & 0 & 0 & 1 & 0 & 0 & 0 & 0 \\
0 & 0 & 1 & 0 & 0 & 0 & 0 & 0 \\
0 & 0 & 0 & 0 & 1 & 0 & 0 & 0 \\
0 & 0 & 0 & 0 & 0 & -1 & 0 & 0
\end{array}\right) \quad \tau=\left(\begin{array}{rrrrrrrr}
1 & 0 & 0 & 0 & 0 & 0 & 0 & 0 \\
0 & 1 & 0 & 0 & 0 & 0 & 0 & 0 \\
0 & 0 & 1 & 0 & 0 & 0 & 0 & 0 \\
0 & 0 & 0 & 1 & 0 & 0 & 0 & 0 \\
0 & 0 & 0 & 0 & 0 & 0 & 0 & -1 \\
0 & 0 & 0 & 0 & 0 & 0 & 1 & 0 \\
0 & 0 & 0 & 0 & 0 & -1 & 0 & 0 \\
0 & 0 & 0 & 0 & 1 & 0 & 0 & 0
\end{array}\right)
$$


Es ist

$$
\sigma \hat{t}_{r, s} \sigma^{-1}=\hat{t}_{r+s,-r},
$$

die Drehung $\nu(\sigma)$ bildet somit $-a^{\vee}$ auf $-a^{\vee}+b^{\vee}$ und $-b^{\vee}$ auf $-a^{\vee}$ ab. Nun hat $\sigma$ die Ordnung 6 , und weiter ist

$$
\tau \hat{t}_{r, s} \tau^{-1}=\hat{t}_{r,-r-s} ;
$$

somit bildet $\nu(\tau)$ ebenfalls $-a^{\vee}$ auf $-a^{\vee}+b^{\vee}$, aber $-b^{\vee}$ auf $b^{\vee}$ ab. Damit ist die Abbildung $\nu$ eindeutig identifiziert.

In der folgenden ersten Abbildung sind im linken Teil die Fixhyperebenen zu den Spiegelungselementen der Weylgruppe sowie die Punkte des von $a^{\vee}$ und $b^{\vee}$ ganzzahlig aufgespannten Gitters eingezeichnet. Im rechten Teil ist der Dualraum so gezeichnet, dass Orthogonalität bezüglich der kanonischen Paarung in offensichtlicher Weise entscheidbar ist. Wählt man im Dualraum $\alpha:=2 a+b$ und $\beta:=-a$, so hat man mit $\alpha, \alpha+\beta, 2 \alpha+3 \beta, \alpha+2 \beta, \alpha+3 \beta$ und $\beta$ sowie ihren Negativen als den zwölf Normalvektoren zu den $H_{\tau \sigma^{i}}$ das bekannte Wurzelsystem vom Typ $G_{2}$.
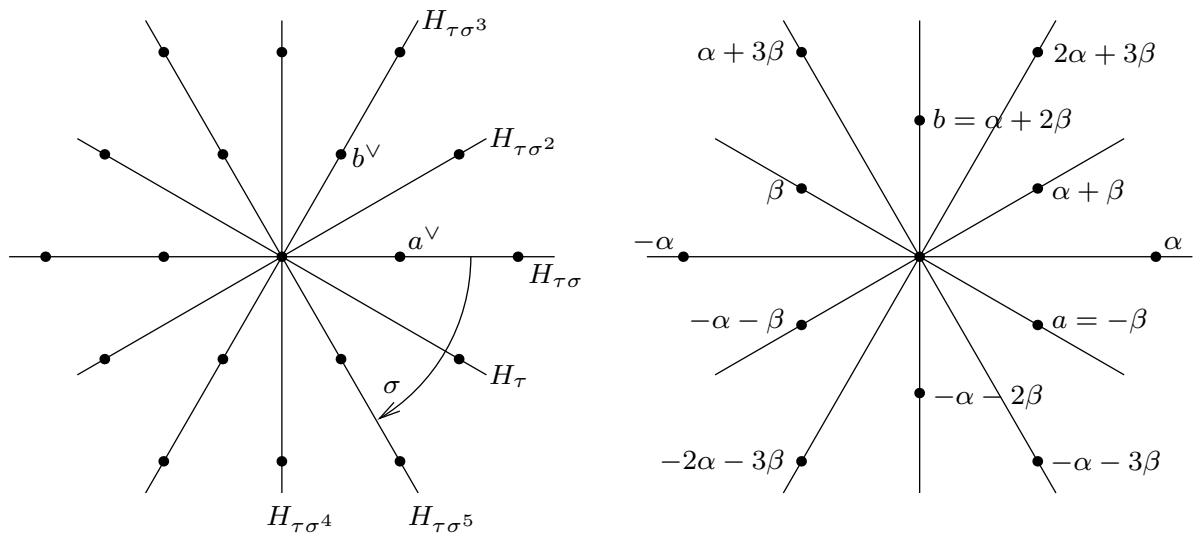

In der nächsten Abbildung ist ein Ausschnitt des vollständigen Systems der Spiegelungshyperebenen der affinen Weylgruppe dargestellt. Dieses ergibt sich aus der Translation der Hyperebenen durch den Nullpunkt mittels der Verschiebung durch die Toruselemente. Nach Konstruktion ist es auch das System aller Hyperebenen $H$, bei denen es eine Wurzel $\theta$ aus dem obigen Wurzelsystem gibt, für die $\omega(\theta(H))=$ const. $\in \mathbb{Z}$ gilt. 


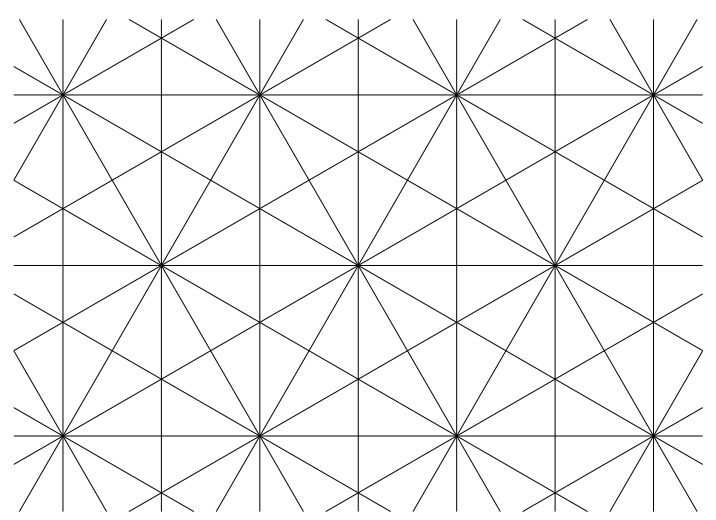

Es ist sofort einsichtig, dass der Abschluss eines jeden kleinen Dreiecks (das im Übrigen die Winkelgrößen $30^{\circ}, 60^{\circ}$ und $90^{\circ}$ besitzt) einen Fundamentalbereich für die Operation von $N(S) / S_{\mathcal{O}}$ darstellt.

\subsection{Die Verbindung Normen - Apartments}

Von besonderem Interesse in dieser Arbeit sind die Normen auf den Oktaven, die sich in Verbindung mit einem festen Apartment ergeben.

Eine Norm kann dadurch beschrieben werden, dass man die durch sie definierte - oder zu definierende - Einheitskugel angibt, hier zunächst verbunden mit der Maßgabe, dass von der zur Norm gehörenden diskreten Bewertung nur ganzzahlige Werte angenommen werden. Bei den Oktaven bietet sich für eine erste solche Norm die Maximalordnung

$$
\Omega:=\left(\begin{array}{ll}
\mathcal{O} & \mathcal{O} \\
\mathcal{O} & \mathcal{O}
\end{array}\right)+\left(\begin{array}{ll}
\mathcal{O} & \mathcal{O} \\
\mathcal{O} & \mathcal{O}
\end{array}\right) \boldsymbol{a}
$$

an.

Die Operation von $Z(S)$ auf der Maximalordnung verändert entsprechend die Normen, die damit auf den von $Z(S)$ erreichbaren Gitterpunkten festgelegt sind.

Beispielhaft sei berechnet:

$$
\hat{t}_{1,0}(\Omega)=\left(\begin{array}{cc}
\mathcal{O} & \pi \mathcal{O} \\
\pi^{-1} \mathcal{O} & \mathcal{O}
\end{array}\right)+\left(\begin{array}{cc}
\mathcal{O} & \pi \mathcal{O} \\
\pi^{-1} \mathcal{O} & \mathcal{O}
\end{array}\right) \boldsymbol{a}
$$

diese Einheitskugel gehört nach Definition von $\nu$ somit zum Punkt $-a^{\vee}$. Entsprechend gehört

$$
\hat{t}_{0,1}(\Omega)=\left(\begin{array}{cc}
\mathcal{O} & \mathcal{O} \\
\mathcal{O} & \mathcal{O}
\end{array}\right)+\left(\begin{array}{cc}
\pi \mathcal{O} & \pi \mathcal{O} \\
\pi^{-1} \mathcal{O} & \pi^{-1} \mathcal{O}
\end{array}\right) \boldsymbol{a}
$$

zum Punkt $-b^{\vee}$ und

$$
\hat{t}_{1,-1}(\Omega)=\left(\begin{array}{cc}
\mathcal{O} & \pi \mathcal{O} \\
\pi^{-1} \mathcal{O} & \mathcal{O}
\end{array}\right)+\left(\begin{array}{cc}
\pi^{-1} \mathcal{O} & \mathcal{O} \\
\mathcal{O} & \pi \mathcal{O}
\end{array}\right) \boldsymbol{a}
$$


zum Punkt $-a^{\vee}+b^{\vee}$.

Zur Verdeutlichung der Lagen der jeweiligen Normen/Einheitskugeln sind in der folgenden Abbildung einige Einheitskugeln zu Punkten im Standardapartment gezeigt, also in dem Apartment zum Standardtorus.

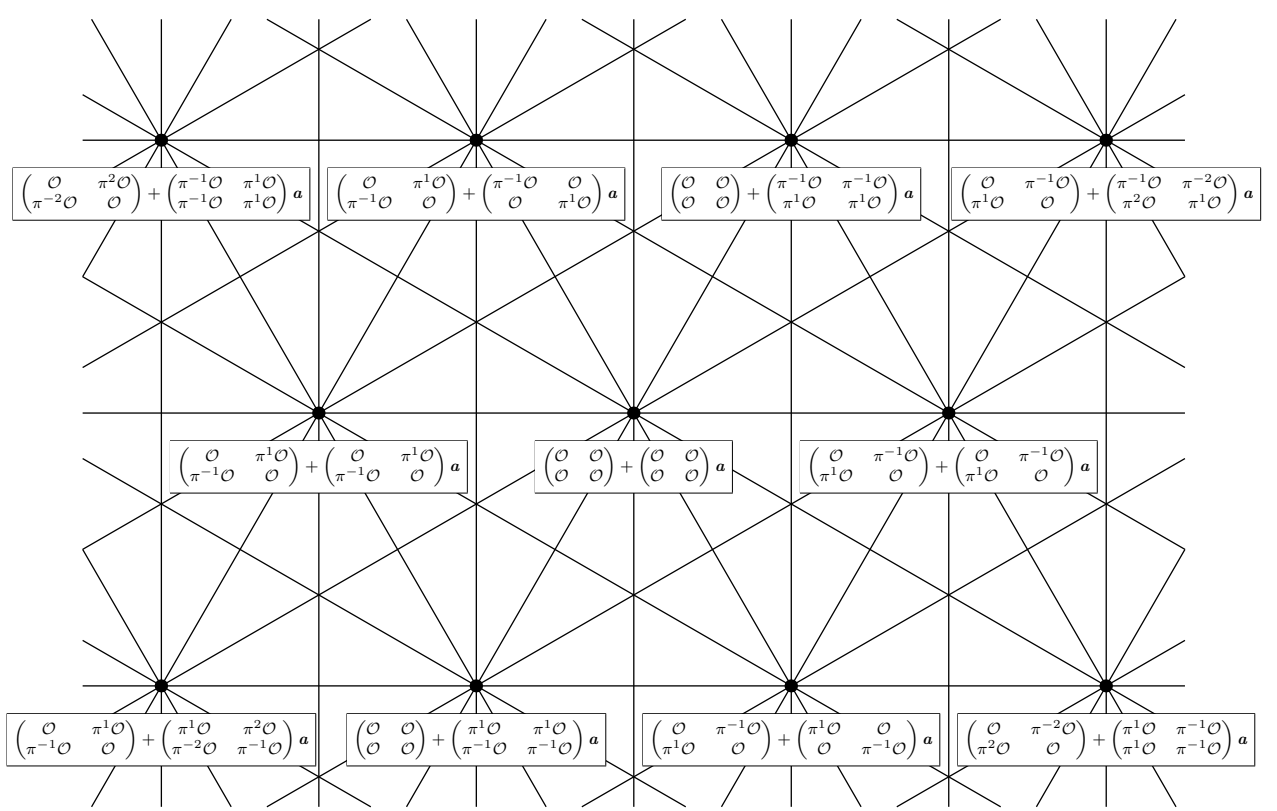

Die so ausgezeichneten Punkte, die auch als diejenigen Punkte beschrieben werden können, durch die von jeder Parallelitätsklasse von Spiegelungshyperebenen ein Exemplar hindurchgeht, heißen spezielle Punkte. Nach [GY03, Kap. 7] sind die zugehörigen Normen genau die Normen mit ganzzahligen Werten.

Die Normen können auf dem Apartment linear interpoliert werden, da die gewählte Basis eine zerfallende Basis (,splitting basis“ - siehe dazu GY03, Abschnitt 2] beziehungsweise Definition 8.2 hier) für alle Normen ist.

Illustriert sei dies an einem Beispiel: In der Abbildung auf Seite 45 sind die Bewertungen von $e_{12}$ an verschiedenen Stellen des Apartments angegeben.

Dargestellt wurden in diesem Abschnitt die Normen, die mit dem Standardtorus in Beziehung stehen. Beim Wechsel in ein anderes Apartment algebraisch zum Beispiel durch Konjugation mit einem Nicht-Toruselement beschrieben - wird die Darstellung der Einheitskugeln bezüglich der Standardbasis komplizierter; für eine Basis, die dem Apartment angepasst ist, verhält sich das System der Normen auf diesem Apartment aber genau wie im Beispiel. 


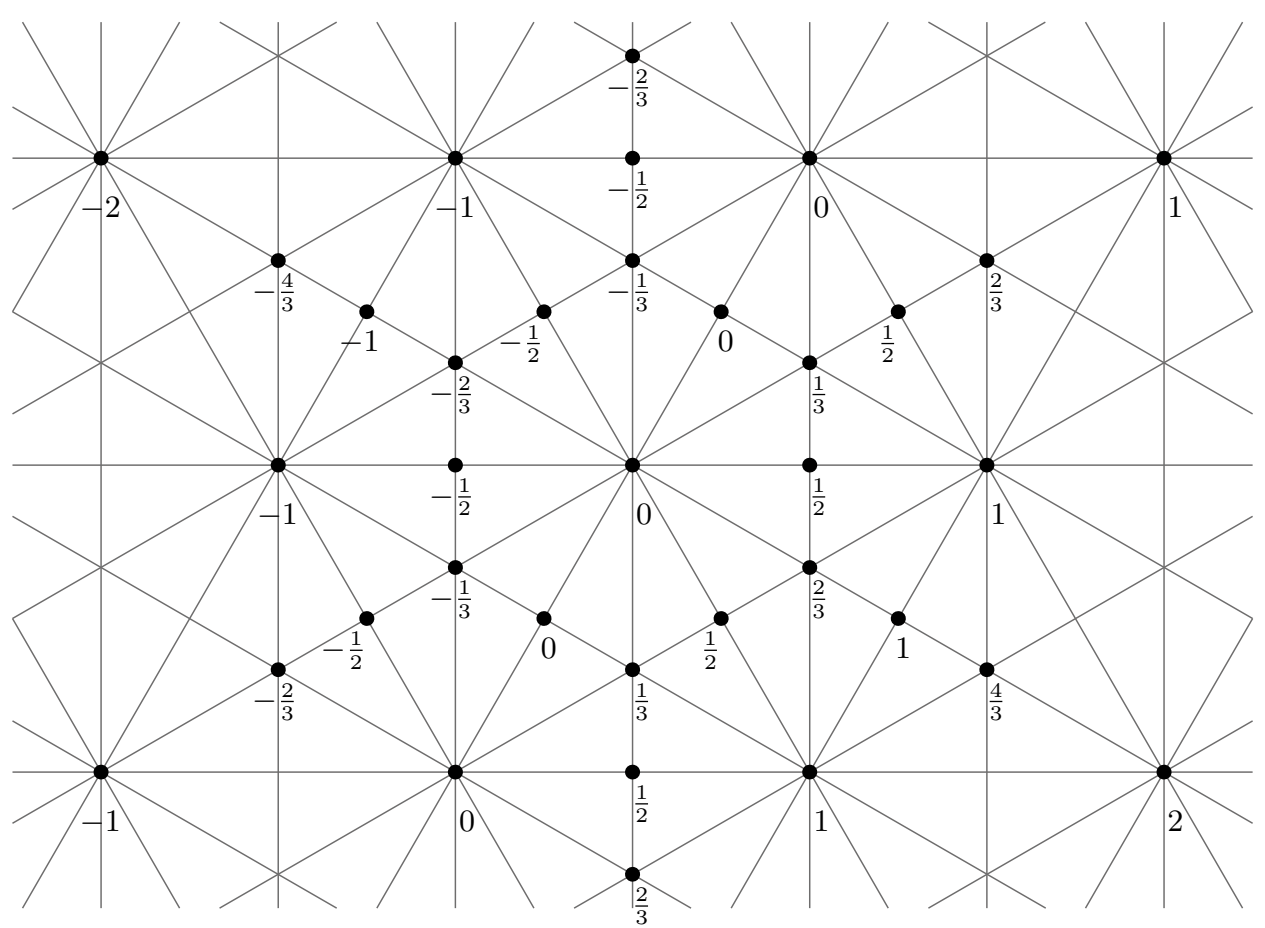

\subsection{Transfer der Wurzeluntergruppen}

Sei $U$ eine Wurzeluntergruppe. Die Stabilisatoruntergruppe für $\Omega$ von $U$ sei mit $U^{\Omega}$ bezeichnet. Offensichtlich ist

$$
U^{\Omega}=U \cap M_{8 \times 8}(\mathcal{O}) .
$$

Die Stabilisatoruntergruppe für eine andere Maximalordnung $\Omega^{\prime}$, die mittels der Translation durch ein Toruselement $\hat{t}_{r, s}$ von $\Omega$ aus erreicht wird, ist offensichtlich $U^{\Omega^{\prime}}:=\hat{t}_{r, s} U^{\Omega} \hat{t}_{r, s}^{-1}$. Aus der Struktur von $\hat{t}_{r, s}$ ergibt sich die folgende allgemeine Beziehung: Es ist $U^{\Omega^{\prime}}=U \cap A_{r, s}$ mit der Matrix $A_{r, s}=$

$$
\left(\begin{array}{cccccccc}
\mathcal{O} & \mathcal{O} & \pi^{-r} \mathcal{O} & \pi^{r} \mathcal{O} & \pi^{-s} \mathcal{O} & \pi^{s} \mathcal{O} & \pi^{-r-s} \mathcal{O} & \pi^{r+s} \mathcal{O} \\
\mathcal{O} & \mathcal{O} & \pi^{-r} \mathcal{O} & \pi^{r} \mathcal{O} & \pi^{-\mathcal{S}} \mathcal{O} & \pi^{s} \mathcal{O} & \pi^{-r-s} \mathcal{O} & \pi^{r+s} \mathcal{O} \\
\pi^{r} \mathcal{O} & \pi^{r} \mathcal{O} & \mathcal{O} & \pi^{2 r} \mathcal{O} & \pi^{r-s} \mathcal{O} & \pi^{r+s} \mathcal{O} & \pi^{-s} \mathcal{O} & \pi^{2 r+s} \mathcal{O} \\
\pi^{-r} \mathcal{O} & \pi^{-r} \mathcal{O} & \pi^{-2 r} \mathcal{O} & \mathcal{O} & \pi^{-r-s} \mathcal{O} & \pi^{-r+s} \mathcal{O} & \pi^{-2 r-s} \mathcal{O} & \pi^{s} \mathcal{O} \\
\pi^{s} \mathcal{O} & \pi^{s} \mathcal{O} & \pi^{-r+s} \mathcal{O} & \pi^{r+s} \mathcal{O} & \mathcal{O} & \pi^{2 s} \mathcal{O} & \pi^{-r} \mathcal{O} & \pi^{r+2 s} \mathcal{O} \\
\pi^{-s} \mathcal{O} & \pi^{-s} \mathcal{O} & \pi^{-r-s} \mathcal{O} & \pi^{r-s} \mathcal{O} & \pi^{-2 s} \mathcal{O} & \mathcal{O} & \pi^{-r-2 s} \mathcal{O} & \pi^{r} \mathcal{O} \\
\pi^{r+s} \mathcal{O} & \pi^{r+s} \mathcal{O} & \pi^{s} \mathcal{O} & \pi^{2 r+s} \mathcal{O} & \pi^{r} \mathcal{O} & \pi^{r+2 s} \mathcal{O} & \mathcal{O} & \pi^{2 r+2 s} \mathcal{O} \\
\pi^{-r-s} \mathcal{O} & \pi^{-r-s} \mathcal{O} & \pi^{-2 r-s} \mathcal{O} & \pi^{-s} \mathcal{O} & \pi^{-r-2 s} \mathcal{O} & \pi^{-r} \mathcal{O} & \pi^{-2 r-2 s} \mathcal{O} & \mathcal{O}
\end{array}\right)
$$




\section{Weitere Strukturuntersuchungen im Normen- Gebäude}

Sei $V$ ein endlich-dimensionaler Vektorraum über $K$. Zu $K$ gehöre eine $p$-adische Bewertung $v_{p}$, kurz: $v$.

Definition 8.1 Eine Bewertung auf dem Vektorraum $V$ ist eine Abbildung $\omega: V \rightarrow \mathbb{R} \cup\{\infty\}$ mit den Eigenschaften:

- $\omega(x+y) \geq \min \{\omega(x), \omega(y)\}$ für alle $x, y \in V$.

- $\omega(\lambda x)=v(\lambda)+\omega(x)$ für $\lambda \in K$ und $x \in V$.

- $\omega(x)=\infty$ genau dann, wenn $x=0$.

Eine Norm auf $V$ entsteht wie bei $p$-adischer Betrachtung üblich parallel $\mathrm{zu}$ der Bewertung über die Beziehung $\|x\|_{v}=p^{-\omega(x)}$, die noch um einen konstanten Faktor abgeändert werden darf.

Definition 8.2 Eine Basis $\left\{b_{1}, \ldots, b_{n}\right\}$ von $V$ heißt spaltende Basis zu $\omega$, wenn gilt:

$$
\omega\left(\sum_{i} \lambda_{i} b_{i}\right)=\min _{i} \omega\left(\lambda_{i} b_{i}\right) .
$$

(Diese beiden Definitionen sind nach [GY03 formuliert.)

In diesem Abschnitt wird betrachtet, welche Basen der Oktaven für eine gegebene $p$-adische Bewertung spalten.

Da Apartments im Gebäude durch Angabe einer - zu einem Torus gehörenden - Basis definiert sind, nämlich indem ein Apartment alle Punkte bzw. Bewertungen enthält, zu denen die Basis spaltet [GY03, Prop. 8.1], ist diese Untersuchung gleichbedeutend mit der Frage, in welchen Apartments ein gegebener Punkt liegt.

Es wird das Gebäude für eine feste Primstelle $p>0$ betrachtet, außerdem als Grundlage ein Apartment zu einer Basis $B=\left\{b_{1}, \ldots, b_{n}\right\}$.

Ohne Einschränkung sei der betrachtete Punkt $O$ im Gebäude bzw. Apartment zu derjenigen Bewertung gehörend, die den Basiselementen die Bewertung 0 zuordnet. Punkte und Bewertungen seien der Einfachheit halber sprachlich gleichgestellt.

Sei $B^{\prime}=\left\{b_{1}^{\prime}, \ldots, b_{n}^{\prime}\right\}$ eine andere Basis, die bezüglich $O$ spaltet. Die Elemente von $B^{\prime}$ seien dabei - auch dies natürlich ohne Einschränkung - so skaliert, dass ihre Bewertung im Punkt $O$ ebenfalls den Wert 0 hat. Dann ist wegen der Spaltungseigenschaft jedes $b_{i}$ als $\mathcal{O}$-Linearkombination der $b_{j}^{\prime}$ darstellbar, und umgekehrt ist auch jedes $b_{j}^{\prime}$ als $\mathcal{O}$-Linearkombination der $b_{i}$ darstellbar. Daraus ergibt sich, dass die Basiswechselmatrix zwischen $B$ 
und $B^{\prime}$ Einträge aus $\mathcal{O}$ besitzt und ihre Determinante die Bewertung 0 hat, die Matrix also in $\mathcal{O}$ invertierbar ist.

Diese notwendige Eigenschaft ist aber auch hinreichend:

Lemma 8.3 SeiO der Punkt in einem Apartment zu einer Basis B, an dem alle Basiselemente die Bewertung 0 haben, wie oben beschrieben. Dann liegt $O$ genau dann im Apartment zu einer Basis $B^{\prime}$, wenn es eine Skalierung ihrer Basiselemente so gibt, dass der Basiswechsel durch ein Element aus $\mathrm{GL}_{n}(\mathcal{O})$ beschrieben werden kann.

Beweis. Zu zeigen bleibt noch, dass es hinreichend für ein Enthaltensein im Apartment zu $B^{\prime}$ ist, dass der Basiswechsel durch ein Element aus $\operatorname{GL}_{n}(\mathcal{O})$ beschrieben werden kann. Sei eine solche Basis $B^{\prime}$ gegeben. Der Beweis des Lemmas ist erbracht, wenn gezeigt wird, dass $B^{\prime}$ bezüglich $O$ spaltet.

Sei $\sum \lambda_{i}^{\prime} b_{i}^{\prime}=\sum \lambda_{i} b_{i}$ ein beliebiges Element des Raumes. Es gilt mit geeigneten $\eta_{i k}, \mu_{k i} \in \mathcal{O}$, die nach Voraussetzung über die Beschreibung des Basiswechsels sämtlich nichtnegative Bewertungen haben:

$$
\begin{aligned}
\min _{k} v\left(\lambda_{k}\right) & =\omega\left(\sum_{k} \lambda_{k} b_{k}\right) \\
& =\omega\left(\sum_{i} \lambda_{i}^{\prime} b_{i}^{\prime}\right) \\
& =\omega\left(\sum_{i} \lambda_{i}^{\prime} \sum_{k} \eta_{i k} b_{k}\right) \\
& =\min _{k} \omega\left(\sum_{i} \lambda_{i}^{\prime} \eta_{i k} b_{k}\right) \\
& =\min _{k} v\left(\sum_{i} \lambda_{i}^{\prime} \eta_{i k}\right) \\
& \geq \min _{i} v\left(\lambda_{i}^{\prime}\right) \\
& =\min _{i} v\left(\sum_{k} \lambda_{k} \mu_{k i}\right) \\
& \geq \min _{k} v\left(\lambda_{k}\right) .
\end{aligned}
$$

Damit muss Gleichheit herrschen, insbesondere gilt

$$
\omega\left(\sum_{i} \lambda_{i}^{\prime} b_{i}^{\prime}\right)=\min _{i} v\left(\lambda_{i}^{\prime}\right)=\min _{i} \omega\left(\lambda_{i}^{\prime} b_{i}^{\prime}\right)
$$

wegen $\omega\left(b_{i}^{\prime}\right)=0$, und somit spaltet $B^{\prime}$ bezüglich $O$. 
Korollar 8.4 In Verallgemeinerung von Lemma 8.3 gilt: Zwei Apartments zu Basen $B$ und $B^{\prime}$ haben genau dann einen Schnittbereich, der einen speziellen Punkt enthält, wenn es Skalierungen der Basiselemente beider Basen so gibt, dass der Basiswechsel durch ein Element aus $\mathrm{GL}_{n}(\mathcal{O})$ beschrieben werden kann.

Beispiel 8.5 Hier und bei den nachfolgenden großen Matrizen sind die Nullen durch Punkte ersetzt, um die Übersichtlichkeit zu erhöhen. - Es werde wieder konkret die Automorphismengruppe der Oktaven betrachtet. Konjugation des Standardtorus

$$
T=\left\{\operatorname{diag}\left(1,1, f, f^{-1}, g, g^{-1}, f g,(f g)^{-1}\right) \mid f, g \in K^{*}\right\}
$$

mit der Matrix

$$
A:=\left(\begin{array}{cccccccc}
1 & \cdot & \cdot & \cdot & \cdot & \cdot & \cdot & \cdot \\
\cdot & 1 & \cdot & \cdot & \cdot & \cdot & \cdot & \cdot \\
\cdot & \cdot & 1 & \cdot & \cdot & \cdot & \cdot & b \\
\cdot & \cdot & \cdot & 1 & \cdot & \cdot & \cdot & \cdot \\
\cdot & \cdot & \cdot & \cdot & 1 & \cdot & \cdot & \cdot \\
\cdot & \cdot & \cdot & \cdot & \cdot & 1 & \cdot & \cdot \\
\cdot & \cdot & \cdot & . & \cdot & \cdot & 1 & \cdot \\
\cdot & \cdot & \cdot & \cdot & \cdot & \cdot & \cdot & \\
\cdot & \cdot & \cdot & \cdot & \cdot & \cdot & \cdot & 1
\end{array}\right)
$$

liefert den Torus

$$
\left\{\left(\begin{array}{cccccccc}
1 & \cdot & \cdot & \cdot & \cdot & \cdot & \cdot & \cdot \\
\cdot & 1 & \cdot & \cdot & \cdot & \cdot & \cdot & \cdot \\
\cdot & \cdot & f & \cdot & \cdot & \cdot & \cdot & b\left(\frac{1}{f g}-f\right) \\
\cdot & \cdot & \cdot & \frac{1}{f} & \cdot & \cdot & \cdot & \cdot \\
\cdot & \cdot & \cdot & \cdot & g & \cdot & \cdot & \cdot \\
\cdot & \cdot & \cdot & \cdot & \cdot & \frac{1}{g} & \cdot & \cdot \\
\cdot & \cdot & \cdot & b\left(\frac{1}{f}-f g\right) & \cdot & \cdot & f g & \cdot \\
\cdot & \cdot & \cdot & \cdot & \cdot & \cdot & \cdot & \frac{1}{f g}
\end{array}\right) \mid f, g \in K^{*}\right\} .
$$

Dieser hat zur Basis

$$
B_{b}^{\prime}:=\left\{e_{11}, e_{22}, e_{12}, e_{21}+b \cdot e_{12} \boldsymbol{a}, e_{11} \boldsymbol{a}, e_{22} \boldsymbol{a}, e_{12} \boldsymbol{a}, e_{21} \boldsymbol{a}+b \cdot e_{12}\right\}
$$

Diagonalgestalt (denn $A$ kann als Basiswechselmatrix aufgefasst werden).

Ein Basiswechsel von der Standardbasis zu einer geeigneten Skalierung von $B_{b}^{\prime}$ ist genau dann in $\mathcal{O}$ machbar, wenn $A \in \mathrm{GL}_{8}(\mathcal{O})$ ist. Dies ist offensichtlich genau dann der Fall, wenn $v(b) \geq 0$ gilt. Genau dann ist folglich das Standardgitter Element des Apartments zu $B_{b}^{\prime}$. 
Ein beliebiger anderer Punkt des Standardapartments ist allein durch die Bewertungen $r=v(f)$ und $s=v(g)$ der Parameter des dorthin transferierenden Toruselements gekennzeichnet. Alle Überlegungen im Folgenden betrachten dementsprechend auch nur die Bewertungen der Koeffizienten bzw. Matrixeinträge. Noch mögliche Faktoren aus $\mathcal{O} / \pi \mathcal{O}$ werden ignoriert, wobei $\pi$ ein uniformisierendes Element von $\mathcal{O}$ sei.

Von der Basis

$$
\left\{e_{11}, e_{22}, \pi^{r} e_{12}, \pi^{-r} e_{21}, \pi^{s} e_{11} \boldsymbol{a}, \pi^{-s} e_{22} \boldsymbol{a}, \pi^{r+s} e_{12} \boldsymbol{a}, \pi^{-r-s} e_{21} \boldsymbol{a}\right\}
$$

ist ein über $\mathcal{O}$ definierter Wechsel zu einer geeigneten Skalierung der Basis $B_{b}^{\prime}$ wegen der speziellen Struktur von $A$ nur zur Basis

$$
\begin{aligned}
& \tilde{B}_{b}^{\prime}:=\left\{e_{11}, e_{22},\right. \pi^{r} e_{12}, \pi^{-r}\left(e_{21}+b \cdot e_{12} \boldsymbol{a}\right), \\
&\left.\pi^{s} e_{11} \boldsymbol{a}, \pi^{-s} e_{22} \boldsymbol{a}, \pi^{r+s} e_{12} \boldsymbol{a}, \pi^{-r-s}\left(b \cdot e_{12}+e_{21} \boldsymbol{a}\right)\right\}
\end{aligned}
$$

möglich, denn die Determinante von $A$ ist gleich dem Produkt der Diagonaleinträge, daher müssen bei dem zu bestimmenden Basiswechsel dort Einsen stehen. Möglich ist ein solcher Wechsel daher genau dann, wenn

$$
-r+v(b) \geq(r+s)
$$

(damit der Koeffizient von $\pi^{r+s} e_{12} \boldsymbol{a}$ in der Darstellung des 4. Elements von $\tilde{B}_{b}^{\prime}$ aus $\mathcal{O}$ ist) und

$$
-r-s+v(b) \geq r
$$

(damit der Koeffizient von $\pi^{r} e_{12}$ in der Darstellung des 8. Elements von $\tilde{B}_{b}^{\prime}$ aus $\mathcal{O}$ ist) gilt.

Beide Bedingungen sind äquivalent zu

$$
2 r+s \leq v(b)
$$

Damit ergibt sich, dass sich die Apartments zu $B$ und $B_{b}^{\prime}$ genau in einer Halbebene schneiden.

Zur Verdeutlichung sei der Schnittbereich der Apartments zur Standardbasis und zur Basis $\tilde{B}_{\pi^{2}}$ dargestellt. Es wurde also $b=\pi^{2}$ gewählt. Die angegebenen Koordinaten sind die oben angeführten Bewertungen $r=v(f)$ und $s=v(g)$. Sie sind im Durchschnittsbereich für beide Apartments gleich. 


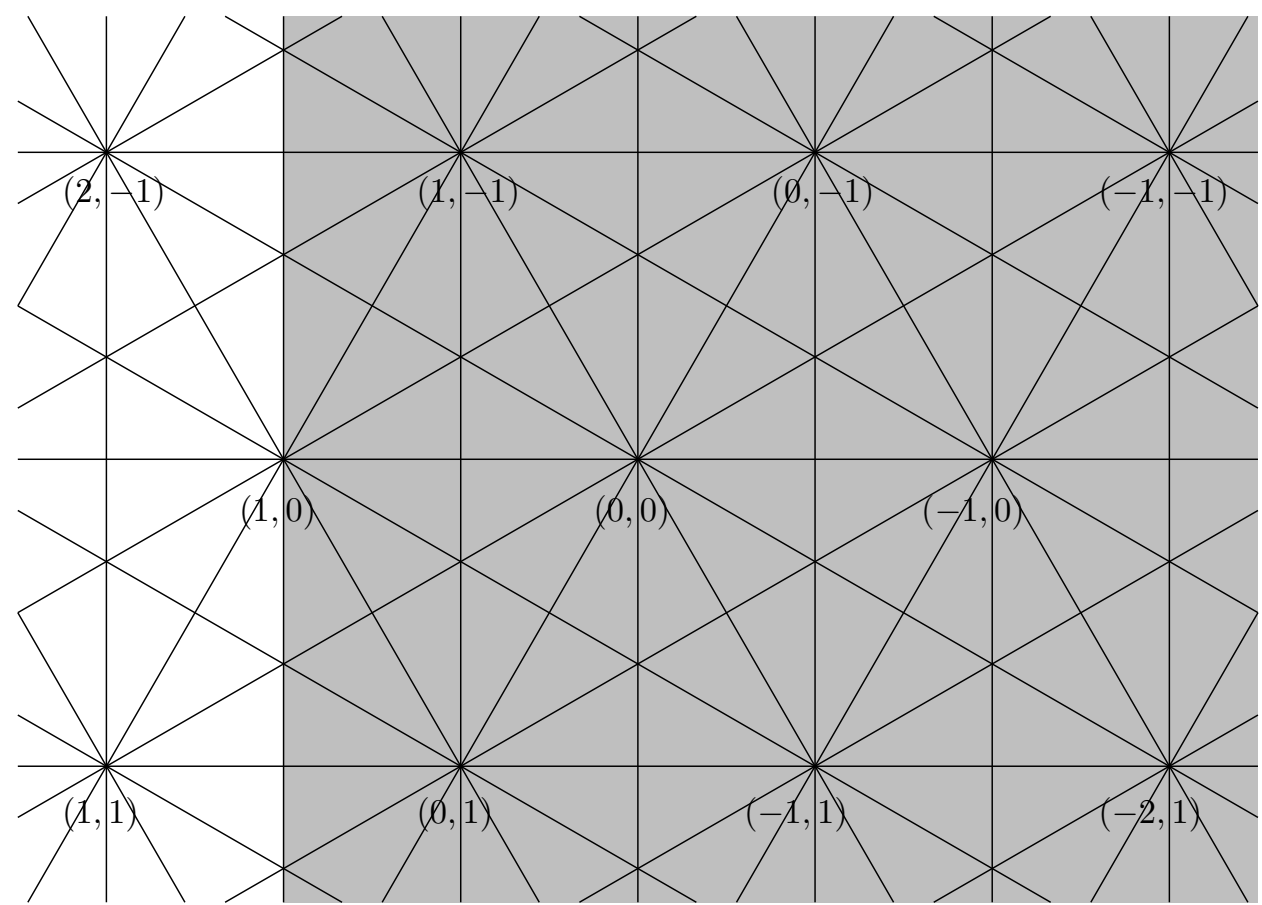

An dieser Stelle sei für Charaktere eine übersichtliche Kurznotation eingeführt. Der Charakter, der $t_{f, g}$ auf $f^{\kappa} g^{\mu}$ abbildet, kann additiv als die Abbildung

$$
\hat{t}_{r, s} \mapsto \kappa r+\mu s
$$

geschrieben werden. Hierfür stehe noch kürzer das Symbol

$$
[\kappa r+\mu s] .
$$

Bemerkung 8.6 Dass das eben betrachtete A ein Element der Wurzeluntergruppe zum Charakter $[2 r+s]$ ist und gleichzeitig der Schnittbereich der beiden Apartments durch die Bedingung $2 r+s \leq v(b)$ oder auch in Wurzelschreibweise: durch $\omega\left(\alpha\left(t_{f, g}\right)\right) \leq v(b)$ bzw. $\left\langle\alpha, \nu\left(t_{f, g}\right)\right\rangle \geq-v(b)$ gegeben ist, ist kein Zufall. Ein Element der Wurzeluntergruppe zu einem Charakter $[\kappa r+\mu s]$ darf außerhalb der Diagonalen nur dort Einträge haben, wo sich beim Konjugieren mit einem Toruselement die Exponenten von $f$ und $g z u \kappa$ bzw. $\mu$ (oder zu Vielfachen mit demselben ganzzahlig-positiven Faktor) addieren. Diese Exponenten ergeben sich an der Position $(i, j)$ aus der Differenz der Exponenten des $i$-ten und des $j$-ten Diagonaleintrags des Toruselements. (Der zweite Eintrag wird negativ gezählt, weil er über das Inverse wirkt.)

Bei einer neuen Skalierung der Basis werden die Basiselemente modulo $\mathcal{O}$ mit dem Toruselement $\operatorname{diag}\left(1,1, \pi^{r}, \pi^{-r}, \pi^{s}, \pi^{-s}, \pi^{r+s}, \pi^{-r-s}\right)$ multipliziert. Ein Nicht-Diagonaleintrag mit Parameter $b$ an der Position $(i, j)$ in der $M a-$ trix, die die Basis des zweiten Apartments darstellt, sorgt dafür, dass der 
j-te Basisvektor des zweiten Apartments eine echte Linearkombination aus der Basis des ersten darstellt. In dem an der neuen Position im Apartment $z u$ untersuchenden Basiswechsel ist der i-te Basisvektor des ersten Apartments mit dem zugehörigen $i$-ten Faktor des Toruselements skaliert worden. In der Linearkombination wurde dieser Term jedoch mit dem Faktor zum $j$-ten Basisvektor skaliert. Daher muss v(b) mindestens so groß wie die Differenz der Bewertungen der Skalierungsfaktoren zum $i$-ten und zum $j$-ten Basisvektor sein. Genau diese Differenz ist (zumindest als Formel) die oben bereits bestimmte.

Die eben geschilderte Beobachtung erleichtert die Bestimmung der weiteren Schnittbereiche. Wie im ersten Beispiel wird der Standardtorus mit einem Element einer Wurzeluntergruppe konjugiert. Die Konjugation kann direkt überprüft werden oder über folgende Rechnung: Sei $U_{\chi}(b)$ ein Element einer Wurzeluntergruppe zum Charakter $\chi$ und mit Parameterwert $b, t_{f, g}=\operatorname{diag}\left(1,1, f, f^{-1}, g, g^{-1}, f g,(f g)^{-1}\right)$ ein Element des Standardtorus. $\mathrm{Zu}$ berechnen ist

$$
\begin{aligned}
U_{\chi}(b) t_{f, g} U_{\chi}^{-1}(b) & =U_{\chi}(b) t_{f, g} U_{\chi}(-b) t_{f, g}^{-1} t_{f, g} \\
& =U_{\chi}(b) U_{\chi}\left(\chi\left(t_{f, g}\right) \cdot(-b)\right) t_{f, g} \\
& =U_{\chi}\left(b \cdot\left(1-\chi\left(t_{f, g}\right)\right)\right) \cdot t_{f, g} .
\end{aligned}
$$

Im ersten Beispiel ist die Wurzel $\chi=\alpha$, das heißt es ist $\chi\left(t_{f, g}\right)=f^{2} g$, der neue Torus wird daher beschrieben durch $U_{\alpha}\left(b \cdot\left(1-f^{2} g\right)\right) \cdot T$.

Damit kann man nun die Rechnungen durchführen, mit deren Hilfe sich die weiteren Schnittbeschreibungen ergeben. Wegen ihrer Länge sind sie in Anhang A.1 zu finden.

Bildlich kann man sich die gerade beschriebene Operation eines Wurzelgruppenelements durch Konjugation auf einem gegebenen Torus so vorstellen, dass das Apartment zum neu entstehenden Torus durch Zur-Seite-Falten an einer geeigneten Geraden aus dem Apartment zum gegebenen Torus entsteht. Diese Gerade ist orthogonal zu der Wurzel, die zu der Wurzeluntergruppe gehört, und der gemeinsame Bereich der beiden Apartments liegt in positiver Richtung bezüglich der Wurzel.

Beim Hintereinanderausführen mehrerer solcher „Faltungen“ kann man sich leicht bei der Reihenfolge bzw. bei der Betrachtungsweise vertun - daher sei hier als Regel angeführt: Konjugationen von außen führen die Faltung (als neue/letzte) am Standardapartment durch. Eine neu eingefügte Konjugation um die Torusmatrix in der Mitte herum sorgt für eine Faltung aus der Sicht des bereits veränderten Apartments. (In vielen Fällen sind die Auswirkungen auf das interessierende Teilergebnis sicherlich zu vernachlässigen. 
Im Beispiel unten ist jedoch erkennbar, dass die Reihenfolge der beiden Faltungen korrekt gewählt werden muss.)

Es seien $A^{\prime}$ und $A^{\prime \prime}$ zwei Apartments, die aus dem Standardapartment durch Falten bezüglich derselben Wurzel entstehen, und zwar mit Parametern $b^{\prime}$ und $b^{\prime \prime}$ (in der Beschreibung wie beim Beispiel 8.5) so, dass $v\left(b^{\prime}\right)=v\left(b^{\prime \prime}\right)$ ist. Daraus ergibt sich zum einen, dass $A^{\prime}$ und $A^{\prime \prime}$ den gleichen Schnitt mit dem Standardapartment besitzen. Weiter gewinnt man $A^{\prime \prime}$ aus $A^{\prime}$ durch eine Faltung derselben Art, und der Parameter hierfür ist $b^{\prime \prime}-b^{\prime}$. Das bedeutet: $A^{\prime}$ und $A^{\prime \prime}$ laufen unmittelbar an der „Faltkante“ bezüglich des Standardapartments genau dann noch gemeinsam weiter, wenn $v\left(b^{\prime \prime}-b^{\prime}\right)>v\left(b^{\prime}\right)$ gilt.

Beispiel 8.7 Um das Prinzip vorzuführen, sei hier einmal ausgerechnet, welche Basis sich beim nacheinander ausgeführten „Falten“ bezüglich $-\alpha$ mit Parameter $c$ und oBdA $v(c)=0$ (das ist also ein Falten genau auf $H_{\tau \sigma^{4}}$, vgl. Zeichnung auf Seite 42 und bezüglich $\alpha$ mit Parameter $b$ ergibt. Das Falten sei dabei jeweils vom Bildapartment aus beschrieben. Man berechnet mit $t_{f, g}=\operatorname{diag}\left(1,1, f, f^{-1}, g, g^{-1}, f g,(f g)^{-1}\right)$ (die Rechnung ist in Anhang A.2 ausgeführt):

$$
\begin{aligned}
& U_{-\alpha}(c) \cdot U_{\alpha}(b) \cdot t_{f, g} \cdot U_{\alpha}^{-1}(b) \cdot U_{-\alpha}^{-1}(c)=
\end{aligned}
$$

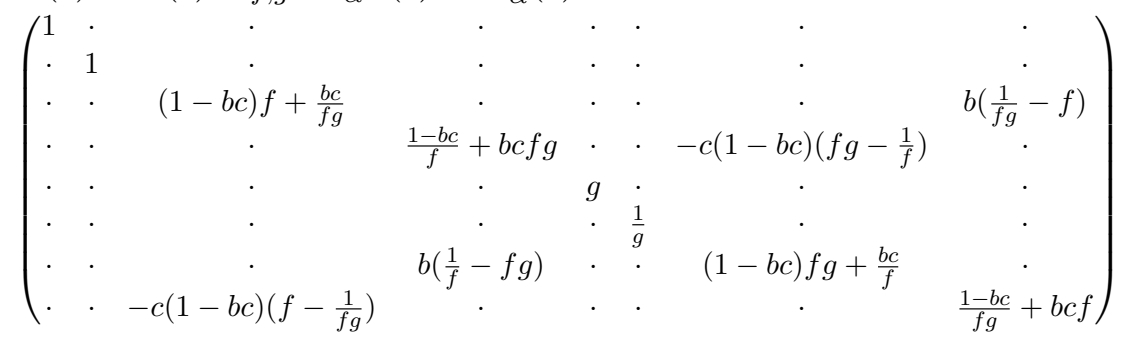

Da Gebäude zusammenziehbar sind, kann nach den obigen Überlegungen zum Abbildungsverhalten für $v(b)<0$ der Schnitt des zum neu gebildeten Torus gehörenden Apartments mit dem Standardapartment nur leer sein. Dementsprechend ist von keinem Punkt des Standardapartments ein über $\mathcal{O}$ definierter Basiswechsel zu einem Punkt des Apartments zu der Basis möglich, die durch die folgende Matrix dargestellt wird. Sie stellt die Situation für $b=\pi$ und $c=1$ dar.

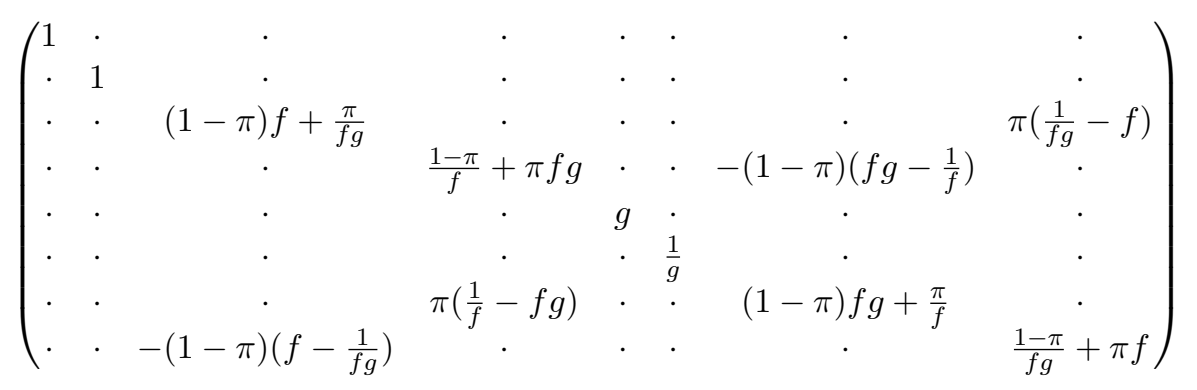

Für $v(b)>0$ bekommt man hingegen einen streifenförmigen Schnittbereich. 
Für $v(b)=0$ ist der Schnittbereich mindestens die Gerade $H_{\tau \sigma^{4}}$ oder sogar ein größerer Bereich in Richtung $\alpha$. Für $b c=1$ ergibt sich beispielsweise als Darstellung des Apartments:

$$
\begin{aligned}
& \left(\begin{array}{cccccccc}
1 & \cdot & \cdot & \cdot & \cdot & \cdot & \cdot & \cdot \\
\cdot & 1 & \cdot & \cdot & \cdot & \cdot & \cdot & \cdot \\
\cdot & \cdot & \frac{1}{f g} & \cdot & \cdot & \cdot & \cdot & b\left(\frac{1}{f g}-f\right) \\
\cdot & \cdot & \cdot & f g & \cdot & \cdot & \cdot & \cdot \\
\cdot & \cdot & \cdot & \cdot & g & \cdot & \cdot & \cdot \\
\cdot & \cdot & \cdot & \cdot & \cdot & \frac{1}{g} & \cdot & \cdot \\
\cdot & \cdot & \cdot & b\left(\frac{1}{f}-f g\right) & \cdot & \cdot & \frac{1}{f} & \cdot \\
\cdot & \cdot & \cdot & \cdot & \cdot & \cdot & \cdot & f
\end{array}\right)
\end{aligned}
$$

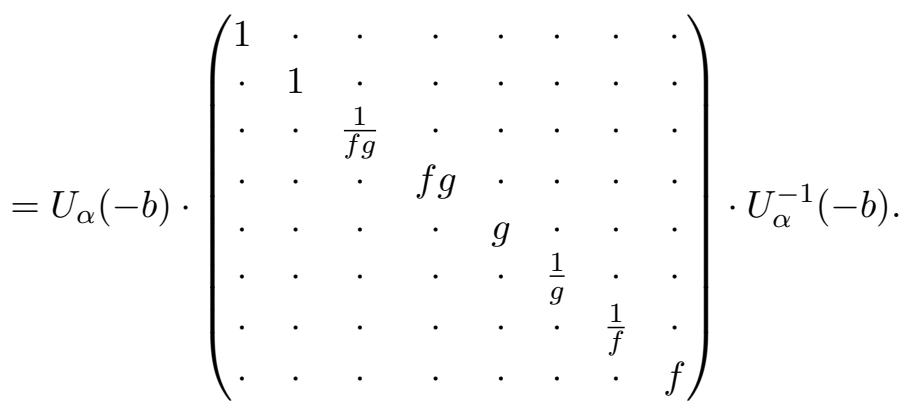

Man erkennt somit, dass hier als Schnittbereich genau die Halbebene rechts der Normalen $\mathrm{zu} \alpha$ vorliegt und dass, wie auch nicht anders zu erwarten war, das zweimal umgefaltete Apartment anders parametrisiert ist als das Standardapartment.

Für $0<v(b c-1)<\infty$ ergibt sich entsprechend ein streifenförmiger Schnittbereich.

Durch Hintereinanderausführen mehrerer Faltungen in verschiedenen Richtungen erhält man leicht ein Apartment, das einen endlichen Schnittbereich mit dem Standardapartment besitzt. Solange eine neue Faltung so ausgeführt wird, dass wenigstens ein Teil des bisherigen Schnittbereichs erhalten bleibt, ist klar, dass dieser nicht wieder größer werden kann, da Schnitte von Apartments konvex sind.

Auch hier sei ein Beispiel gegeben: Die Konjugation des Torus $T$ mit dem Produkt

$$
U_{\alpha}\left(\pi^{3}\right) \cdot U_{\alpha+2 \beta}(\pi) \cdot U_{-2 \alpha-3 \beta}(1) \cdot U_{-\alpha-3 \beta}\left(\pi^{2}\right)
$$

liefert ein Apartment, das den in der Abbildung gezeigten Schnittbereich mit dem Standardapartment hat: 


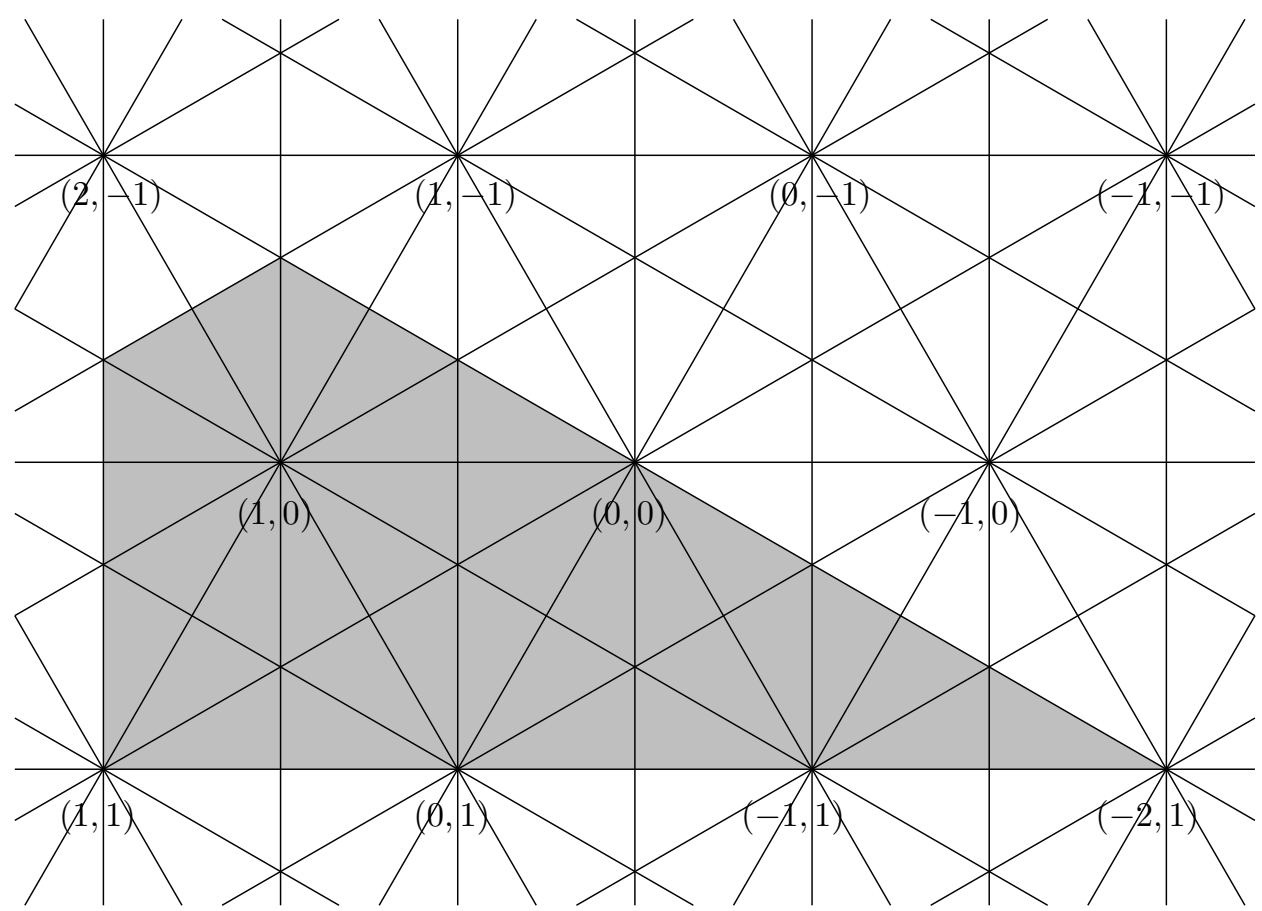




\section{Cartan-Killing-Form und symmetrischer Raum}

\subsection{Die Cartan-Killing-Form}

Für die archimedische Norm ist die Beschreibung des Raums der möglichen Normen etwas anders als für eine nicht-archimedische Norm. Eine mögliche Beschreibung geht von der Cartan-Killing-Form auf der Lie-Algebra $\mathfrak{g}$ zur Automorphismengruppe $G$ der Oktaven aus.

Aus der Gestalt der Wurzeluntergruppen, die in Kapitel 6.2 bestimmt wurden, ergibt sich sofort eine Basis für die Lie-Algebra $\mathfrak{g}$ :

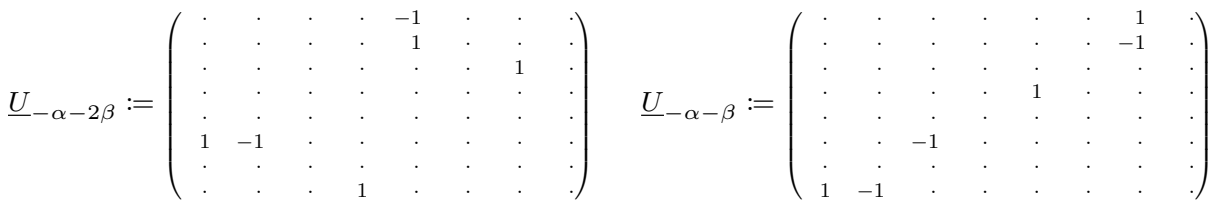

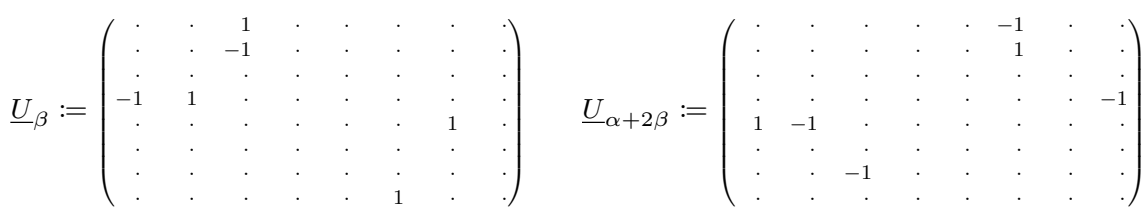

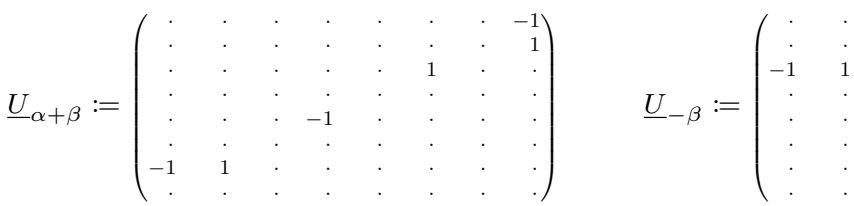

$$
\begin{aligned}
& \underline{U}_{\alpha}:=\left(\begin{array}{cccccccc}
\vdots & \vdots & \vdots & \vdots & \vdots & \vdots & \vdots & \\
\vdots & \vdots & \vdots & \vdots & \vdots & \vdots & \vdots & 1 \\
\vdots & \vdots & \vdots & \vdots & \vdots & \vdots & \vdots & \vdots \\
\vdots & \vdots & \vdots & 1 & \vdots & \vdots & \vdots & \vdots
\end{array}\right) \\
& \underline{U}_{-\alpha-3 \beta}:=\left(\begin{array}{rrrrrrrr}
\vdots & \vdots & \vdots & \vdots & \vdots & \vdots & \vdots & \\
\vdots & \vdots & \vdots & \vdots & -1 & \vdots & \vdots & \\
\vdots & \vdots & \vdots & \vdots & \vdots & \vdots & \vdots & \vdots \\
\vdots & \vdots & \vdots & \vdots & \vdots & \vdots & \vdots & \vdots
\end{array}\right) \\
& \underline{U}_{-2 \alpha-3 \beta}:=\left(\begin{array}{cccccccc}
\vdots & \vdots & \vdots & \vdots & \vdots & \vdots & \vdots & \\
\vdots & \vdots & \vdots & \vdots & \vdots & \vdots & \vdots & \\
\vdots & \vdots & \vdots & \vdots & \vdots & \vdots & \vdots & \vdots \\
\vdots & \vdots & \vdots & \vdots & \vdots & \vdots & -1 & \vdots \\
\vdots & \vdots & & -1 & \vdots & \vdots & \vdots
\end{array}\right) \\
& \underline{U}_{-\alpha}:=\left(\begin{array}{rrrrrrrr}
\vdots & \vdots & \vdots & \vdots & \vdots & \vdots & \vdots & \\
\vdots & \vdots & \vdots & \vdots & \vdots & \vdots & \vdots & \\
\vdots & \vdots & \vdots & \vdots & \vdots & \vdots & -1 & \vdots \\
\vdots & \vdots & \vdots & \vdots & \vdots & \vdots & \vdots & \vdots \\
\vdots & \vdots & -1 & \vdots & \vdots & \vdots & \vdots & \vdots
\end{array}\right) \\
& \underline{U}_{\alpha+3 \beta}:=\left(\begin{array}{cccccccc}
\vdots & \vdots & \vdots & \vdots & \vdots & \vdots & \vdots & \\
\vdots & \vdots & \vdots & \vdots & \vdots & -1 & \vdots & \\
\vdots & \vdots & 1 & \vdots & \vdots & -1 & \vdots & \vdots \\
\vdots & \vdots & \vdots & \vdots & \vdots & \vdots & \vdots & \vdots
\end{array}\right) \\
& \underline{U}_{2 \alpha+3 \beta}:=\left(\begin{array}{cccccccc}
\vdots & \vdots & \vdots & \vdots & \vdots & \vdots & \vdots & \\
\vdots & \vdots & \vdots & \vdots & \vdots & \vdots & \vdots & \\
\vdots & \vdots & \vdots & \vdots & \vdots & \vdots & \vdots & \\
\vdots & \vdots & \vdots & \vdots & \vdots & \vdots & \vdots & \vdots \\
\vdots & \vdots & \vdots & \vdots & \vdots & \vdots & \vdots & \vdots
\end{array}\right)
\end{aligned}
$$

Es wird leicht das Folgende berechnet ${ }^{2}$,

$$
\left[\underline{U}_{\alpha}, \underline{U}_{\alpha}\right]=0
$$

$$
\left[\underline{U}_{\beta}, \underline{U}_{\alpha}\right]=-\underline{U}_{\alpha+\beta}
$$

\footnotetext{
${ }^{2}$ Auch ohne Kenntnis der Struktur einer Lie-Algebra vom Typ $G_{2}$ kann der Rechenaufwand minimiert werden, wenn man Formel (9.1) und die entsprechende Formel für die Spiegelung $\tau$ benutzt - dann verbleiben 9 wesentliche Rechnungen.
} 


$$
\begin{aligned}
{\left[\underline{U}_{\alpha}, \underline{U}_{-\alpha-3 \beta}\right] } & =0 & {\left[\underline{U}_{\beta}, \underline{U}_{-\alpha-3 \beta}\right] } & =\underline{U}_{-\alpha-2 \beta} \\
{\left[\underline{U}_{\alpha}, \underline{U}_{-2 \alpha-3 \beta}\right] } & =\underline{U}_{-\alpha-3 \beta} & {\left[\underline{U}_{\beta}, \underline{U}_{-2 \alpha-3 \beta}\right] } & =0 \\
{\left[\underline{U}_{\alpha}, \underline{U}_{-\alpha}\right] } & =\operatorname{diag}(0,0,-1,1,0,0,-1,1) & {\left[\underline{U}_{\beta}, \underline{U}_{-\alpha}\right] } & =0 \\
{\left[\underline{U}_{\alpha}, \underline{U}_{\alpha+3 \beta}\right] } & =-\underline{U}_{2 \alpha+3 \beta} & {\left[\underline{U}_{\beta}, \underline{U}_{\alpha+3 \beta}\right] } & =0 \\
{\left[\underline{U}_{\alpha}, \underline{U}_{2 \alpha+3 \beta}\right] } & =0 & {\left[\underline{U}_{\beta}, \underline{U}_{2 \alpha+3 \beta}\right] } & =0 \\
{\left[\underline{U}_{\alpha}, \underline{U}_{\beta}\right] } & =\underline{U}_{\alpha+\beta} & {\left[\underline{U}_{\beta}, \underline{U}_{\beta}\right] } & =0 \\
{\left[\underline{U}_{\alpha}, \underline{U}_{\alpha+2 \beta}\right] } & =0 & \left.\underline{U}_{\beta}, \underline{U}_{\alpha+2 \beta}\right] & =-3 \underline{U}_{\alpha+3 \beta} \\
{\left[\underline{U}_{\alpha}, \underline{U}_{\alpha+\beta}\right] } & =0 & {\left[\underline{U}_{\beta}, \underline{U}_{\alpha+\beta}\right] } & =-2 \underline{U}_{\alpha+2 \beta} \\
{\left[\underline{U}_{\alpha}, \underline{U}_{-\beta}\right] } & =0 & {\left[\underline{U}_{\beta}, \underline{U}_{-\beta}\right] } & =\operatorname{diag}(0,0,2,-2,-1,1,1,-1) \\
{\left[\underline{U}_{\alpha}, \underline{U}_{-\alpha-2 \beta}\right] } & =0 & {\left[\underline{U}_{\beta}, \underline{U}_{-\alpha-2 \beta}\right] } & =2 \underline{U}_{-\alpha-\beta} \\
{\left[\underline{U}_{\alpha}, \underline{U}_{-\alpha-\beta}\right] } & =-\underline{U}_{-\beta} & {\left[\underline{U}_{\beta}, \underline{U}_{-\alpha-\beta}\right] } & =3 \underline{U}_{-\alpha}
\end{aligned}
$$

Die Berechnungen der anderen Lie-Klammern ergeben sich aus der offensichtlichen Formel

$$
\left[\sigma^{i} \underline{U}_{\zeta} \sigma^{-i}, \sigma^{i} \underline{U}_{\eta} \sigma^{-i}\right]=\sigma^{i}\left[\underline{U}_{\zeta}, \underline{U}_{\eta}\right] \sigma^{-i}
$$

dabei ist $\sigma$ die auf Seite 38 definierte Drehung aus der Weylgruppe.

Die Cartan-Unteralgebra, also die Lie-Algebra $\mathfrak{h}=\operatorname{Lie}(T)$ zum maximalen Torus, wird erzeugt von

$$
\begin{aligned}
& \underline{h}_{1,0}:=\operatorname{diag}(0,0,1,-1,0, \quad 0,1,-1) \quad \text { und } \\
& \underline{h}_{0,1}:=\operatorname{diag}(0,0,0, \quad 0,1,-1,1,-1) .
\end{aligned}
$$

Weiterführen der Berechnungen liefert mit der Bezeichnung $\underline{\tilde{H}}_{\eta}:=\left[\underline{U}_{\eta}, \underline{U}_{-}\right]$:

$$
\begin{aligned}
& \underline{\tilde{H}}_{\alpha}=\operatorname{diag}(0,0,-1,1,0,0,-1,1) \\
& \underline{\tilde{H}}_{-\alpha-3 \beta}=\operatorname{diag}(0,0,-1,1,1,-1,0,0) \\
& \underline{\tilde{\tilde{H}}}_{-2 \alpha-3 \beta}=\operatorname{diag}(0,0,0,0,1,-1,1,-1) \\
& \underline{\tilde{H}}_{-\alpha}=\operatorname{diag}(0,0,1,-1,0,0,1,-1) \\
& \underline{\tilde{H}}_{\alpha+3 \beta}=\operatorname{diag}(0,0,1,-1,-1,1,0,0) \\
& \underline{\tilde{H}}_{2 \alpha+3 \beta}=\operatorname{diag}(0,0,0,0,-1,1,-1,1) \\
& \underline{\tilde{H}}_{\beta}=\operatorname{diag}(0,0,2,-2,-1,1,1,-1) \\
& \underline{\tilde{H}}_{\alpha+2 \beta}=\operatorname{diag}(0,0,1,-1,-2,2,-1,1) \\
& \underline{\tilde{H}}_{\alpha+\beta}=\operatorname{diag}(0,0,-1,1,-1,1,-2,2) \\
& \underline{\tilde{H}}_{-\beta}=\operatorname{diag}(0,0,-2,2,1,-1,-1,1) \\
& \underline{\tilde{H}}_{-\alpha-2 \beta}=\operatorname{diag}(0,0,-1,1,2,-2,1,-1) \\
& \underline{\tilde{H}}_{-\alpha-\beta}=\operatorname{diag}(0,0,1,-1,1,-1,2,-2)
\end{aligned}
$$

Eventuell bemerkenswert ist, dass für beide Blöcke (links als Indizes die sechs langen Wurzeln, rechts die sechs kurzen Wurzeln) gilt: Falls $\zeta, \eta$ und $\zeta+\eta$ Wurzeln sind, gilt $\underline{\tilde{H}}_{\zeta}+\underline{\tilde{H}}_{\eta}=\underline{\tilde{H}}_{\zeta+\eta}$. Für eine Addition von Elementen von beiden Blöcken gemischt gilt dies jedoch nicht, obwohl immerhin jeweils ein $\underline{\tilde{H}}_{\theta}$ mit einer anderen Wurzel $\theta$ das Ergebnis der Addition ist.

Für die weiteren Schritte benötigt man für jede Wurzel eine Unter-LieAlgebra mit Basis, die isomorph zur $\mathfrak{s l}_{2}$ mit ihrer Standardbasis ist. Das heißt, dass zu jeder Wurzel $\zeta$ Elemente $X_{\zeta}, X_{-\zeta}$ und $H_{\zeta}$ gesucht sind mit 
- $\left[X_{\zeta}, X_{-\zeta}\right]=H_{\zeta}$,

- $\left[H, X_{\zeta}\right]=\zeta(H) X_{\zeta}$ für $H$ aus der Cartan-Unteralgebra $\mathfrak{h}$, wobei $\zeta$ als Linearform auf $\mathfrak{h}$ wirkt, insbesondere muss für die Isomorphie zur $\mathfrak{s l}_{2}$ die Beziehung $\left[H_{\zeta}, X_{\zeta}\right]=2 X_{\zeta}$ gelten.

Selbstverständlich soll weiterhin die Graduierung berücksichtigt werden, also gelten:

- Falls $\zeta, \eta, \zeta+\eta \in \Phi$, dann ist $\left[X_{\zeta}, X_{\eta}\right]=c_{\zeta, \eta} X_{\zeta+\eta}$ mit Strukturkonstanten $c_{\zeta, \eta}$ aus dem Grundkörper.

Die $\underline{U}_{\zeta}$ und die $\underline{\tilde{H}}_{\zeta}$ erfüllen diese Bedingungen fast vollständig (wobei die Wirkung einer Wurzel auf ein Element der Cartan-Unteralgebra implizit über die zweite Bedingung definiert wird), einzig $\zeta\left(\underline{\tilde{H}}_{\zeta}\right)$ ist nicht 2, sondern -2. Daher muss bei jedem Paar von Wurzeln ein Basisvektor noch mit dem Faktor -1 multipliziert werden. Welchen der beiden man jeweils auswählt, ist im Moment nicht von besonderer Bedeutung. Es seien hier jeweils die Basisvektoren zu negativen Wurzeln abgeändert.

Demnach sei

$$
\begin{array}{ll}
X_{\zeta}:=\underline{U}_{\zeta} & \text { für positive } \zeta, \mathrm{d} . \mathrm{h} . \zeta=i \alpha+j \beta \text { mit } i, j \geq 0, \\
X_{\zeta}:=-\underline{U}_{\zeta} & \text { für negative } \zeta, \mathrm{d} . \mathrm{h} . \zeta=k \alpha+l \beta \text { mit } k, l \leq 0, \\
H_{\zeta}:=-\underline{\tilde{H}}_{\zeta} & \text { für alle } \zeta .
\end{array}
$$

Zur leichteren Nachvollziehbarkeit der weiteren Rechnungen seien hier beispielhaft die Zahlenwerte bei der Auswertung von $\alpha$ bzw. $\beta$ auf $\mathfrak{h}$ genannt: Es ist $\alpha=2 h_{1,0}^{*}+h_{0,1}^{*}$ sowie $\beta=-h_{1,0}^{*}$ und damit

$$
\begin{array}{rlrl}
\alpha\left(H_{\alpha}\right) & =2 & 2 & \alpha\left(H_{\beta}\right)=-3 \\
\alpha\left(H_{-\alpha-3 \beta}\right) & =1 & \alpha\left(H_{\alpha+2 \beta}\right) & =0 \\
\alpha\left(H_{-2 \alpha-3 \beta}\right) & =-1 & \alpha\left(H_{\alpha+\beta}\right) & =3 \\
\alpha\left(H_{-\alpha}\right) & =-2 & \alpha\left(H_{-\beta}\right) & =3 \\
\alpha\left(H_{\alpha+3 \beta}\right) & =-1 & \alpha\left(H_{-\alpha-2 \beta}\right) & =0 \\
\alpha\left(H_{2 \alpha+3 \beta}\right) & =1 & \alpha\left(H_{-\alpha-\beta}\right) & =-3 \\
\beta\left(H_{\alpha}\right) & =-1 & & \\
\beta\left(H_{\beta}\right) & =2 \\
\beta\left(H_{-\alpha-3 \beta}\right) & =-1 & \beta\left(H_{\alpha+2 \beta}\right) & =1 \\
\beta\left(H_{-2 \alpha-3 \beta}\right) & =0 & \beta\left(H_{\alpha+\beta}\right) & =-1 \\
\beta\left(H_{-\alpha}\right) & =1 & \beta\left(H_{-\beta}\right) & =-2 \\
\beta\left(H_{\alpha+3 \beta}\right) & =1 & \beta\left(H_{-\alpha-2 \beta}\right) & =-1 \\
\beta\left(H_{2 \alpha+3 \beta}\right) & =0 & \beta\left(H_{-\alpha-\beta}\right) & =1 .
\end{array}
$$


Die Werte für die anderen Wurzeln ergeben sich mit dem gleichen Argument wie oben analog.

Definition 9.1 Für ein solches System von $X_{\eta}, \eta \in \Phi$, und $\mathfrak{h}$ definiert man die

$$
\text { Standard-Involution } \quad \Theta: \mathfrak{g} \rightarrow \mathfrak{g}
$$

wie folgt:

- $\Theta(H):=-H \quad$ für $H \in \mathfrak{h}$,

- $\Theta\left(X_{\eta}\right):=-X_{-\eta}$ für $\eta \in \Phi$.

Diese Definition setzt sich zu einem involutiven Lie-Algebren-Automorphismus von $\mathfrak{g}$ fort.

Auf $\mathfrak{g}$ hat man die Cartan-Killing-Form

$$
(X, Y):=\operatorname{Spur}(\operatorname{ad}(X) \operatorname{ad}(Y)) .
$$

Sie ist invariant in dem Sinne, dass für $X, Y, Z \in \mathfrak{g}$ gilt:

$$
([Z, X], Y)+(X,[Z, Y])=0 .
$$

In Verbindung mit der Standard-Involution erhält man (über $\mathbb{R}$ ) eine symmetrische Bilinearform

$$
(X, Y)_{0}:=-(X, \Theta(Y))
$$

auf $\mathfrak{g}$.

Im hier behandelten konkreten Fall respektiert die Lie-Klammer die Graduierung durch die Wurzelbasis. Daher ist klar, dass die $\operatorname{Spur} \operatorname{von} \operatorname{ad}\left(X_{\zeta}\right) \operatorname{ad}\left(X_{\eta}\right)$ mindestens dann verschwindet, wenn $\zeta \neq-\eta$ ist. Aus den obigen Berechnungen ist umgekehrt Folgendes zu erkennen:

$$
\begin{aligned}
{\left[X_{\alpha},\left[X_{-\alpha}, X_{2 \alpha+3 \beta}\right]\right] } & =\left[\underline{U}_{\alpha},\left[-\underline{U}_{-\alpha}, \underline{U}_{2 \alpha+3 \beta}\right]\right]=X_{2 \alpha+3 \beta} \\
{\left[X_{\alpha},\left[X_{-\alpha}, X_{\alpha+\beta}\right]\right] } & =\left[\underline{U}_{\alpha},\left[-\underline{U}_{-\alpha}, \underline{U}_{\alpha+\beta}\right]\right]=X_{\alpha+\beta} \\
{\left[X_{\alpha},\left[X_{-\alpha}, X_{\alpha}\right]\right] } & =\left[\underline{U}_{\alpha},\left[-\underline{U}_{-\alpha}, \underline{U}_{\alpha}\right]\right]=2 X_{\alpha} \\
{\left[X_{\alpha},\left[X_{-\alpha}, X_{-\beta}\right]\right] } & =\left[\underline{U}_{\alpha},\left[-\underline{U}_{-\alpha},-\underline{U}_{-\beta}\right]\right]=X_{-\beta} \\
{\left[X_{\alpha},\left[X_{-\alpha}, X_{-\alpha-3 \beta}\right]\right] } & =\left[\underline{U}_{\alpha},\left[-\underline{U}_{-\alpha},-\underline{U}_{-\alpha-3 \beta}\right]\right]=X_{-\alpha-3 \beta} \\
{\left[X_{\alpha},\left[X_{-\alpha}, H_{\alpha}\right]\right] } & =\left[\underline{U}_{\alpha},\left[H_{\alpha}, \underline{U}_{-\alpha}\right]\right]=2 H_{\alpha}
\end{aligned}
$$

Weitere Beiträge zur Spur werden nicht geliefert. Damit ist

$$
\left(X_{\zeta}, X_{\zeta}\right)_{0}=\left(X_{\zeta}, X_{-\zeta}\right)=8
$$


für alle langen Wurzeln $\zeta$.

Analog berechnet man

$$
\begin{aligned}
{\left[X_{\beta},\left[X_{-\beta}, X_{-\alpha-2 \beta}\right]\right] } & =\left[\underline{U}_{\beta},\left[-\underline{U}_{-\beta},-\underline{U}_{-\alpha-2 \beta}\right]\right]=3 X_{-\alpha-2 \beta} \\
{\left[X_{\beta},\left[X_{-\beta}, X_{-\alpha-\beta}\right]\right] } & =\left[\underline{U}_{\beta},\left[-\underline{U}_{-\beta},-\underline{U}_{-\alpha-\beta}\right]\right]=4 X_{-\alpha-\beta} \\
{\left[X_{\beta},\left[X_{-\beta}, X_{-\alpha}\right]\right] } & =\left[\underline{U}_{\beta},\left[-\underline{U}_{-\beta},-\underline{U}_{-\alpha}\right]\right]=3 X_{-\alpha} \\
{\left[X_{\beta},\left[X_{-\beta}, X_{\beta}\right]\right] } & =\left[\underline{U}_{\beta},\left[-\underline{U}_{-\beta}, \underline{U}_{\beta}\right]\right]=2 X_{\beta} \\
{\left[X_{\beta},\left[X_{-\beta}, X_{\alpha+3 \beta}\right]\right] } & =\left[\underline{U}_{\beta},\left[-\underline{U}_{-\beta}, \underline{U}_{\alpha+3 \beta}\right]\right]=3 X_{\alpha+3 \beta} \\
{\left[X_{\beta},\left[X_{-\beta}, X_{\alpha+2 \beta}\right]\right] } & =\left[\underline{U}_{\beta},\left[-\underline{U}_{-\beta}, \underline{U}_{\alpha+2 \beta}\right]\right]=4 X_{\alpha+2 \beta} \\
{\left[X_{\beta},\left[X_{-\beta}, X_{\alpha+\beta}\right]\right] } & =\left[\underline{U}_{\beta},\left[-\underline{U}_{-\beta}, \underline{U}_{\alpha+\beta}\right]\right]=3 X_{\alpha+\beta} \\
{\left[X_{\beta},\left[X_{-\beta}, H_{\beta}\right]\right] } & =\left[\underline{U}_{\beta},\left[H_{\beta}, \underline{U}_{-\beta}\right]\right]=2 H_{\beta},
\end{aligned}
$$

und es ergibt sich für alle kurzen Wurzeln $\eta$ :

$$
\left(X_{\eta}, X_{\eta}\right)_{0}=\left(X_{\eta}, X_{-\eta}\right)=24
$$

Für die $H_{\zeta}$ ergibt sich

$$
\left(H_{\zeta}, H_{\zeta}\right)_{0}=-\left(H_{\zeta}, H_{-\zeta}\right)=\left(H_{\zeta}, H_{\zeta}\right)=\sum_{\eta \in \Phi} \eta\left(H_{\zeta}\right)^{2}
$$

damit errechnet sich aus 9.2 und 9.3

$$
\left(H_{\zeta}, H_{\zeta}\right)_{0}=16
$$

für alle langen Wurzeln $\zeta$ und

$$
\left(H_{\zeta}, H_{\zeta}\right)_{0}=48
$$

für alle kurzen Wurzeln $\zeta$.

Dieses Ergebnis ist selbstverständlich in Einklang mit der Formel

$$
\left(X_{\zeta}, X_{\zeta}\right)_{0}=\frac{1}{2}\left(H_{\zeta}, H_{\zeta}\right)_{0}
$$

aus [HSt], Proposition 4.8.

Weiterhin berechnet man

$$
\left(H_{\alpha}, H_{\beta}\right)_{0}=\left(H_{\alpha}, H_{\beta}\right)=\sum_{\eta \in \Phi} \eta(\alpha) \eta(\beta)=-24 .
$$




\subsection{Eigenschaften der Cartan-Killing-Form}

Lemma 9.2 Bezüglich der Cartan-Killing-Form ist die Zerlegung

$$
\mathfrak{g}=\operatorname{Lie}(T) \oplus \bigoplus_{\zeta \in \Phi_{+}}\left(\mathfrak{g}_{\zeta} \oplus \mathfrak{g}_{-\zeta}\right)
$$

orthogonal.

Bezüglich der Form $(\cdot, \cdot)_{0}$ ist die Zerlegung

$$
\mathfrak{g}=\operatorname{Lie}(T) \oplus \bigoplus_{\zeta \in \Phi} \mathfrak{g}_{\zeta}
$$

orthogonal.

Dabei bezeichne $\mathfrak{g}_{\zeta}$ den von $X_{\zeta}$ aufgespannten Raum.

Beweis. Sei $X \in \mathfrak{g}_{\zeta}, Y \in \mathfrak{g}_{\eta}$ und $H \in \mathfrak{h}$. Da die Cartan-Killing-Form invariant ist, gilt

$$
([H, X], Y)+(X,[H, Y])=0 .
$$

Dies kann man aber auch als

$$
\zeta(H) \cdot(X, Y)+\eta(H) \cdot(X, Y)=0
$$

schreiben.

Wenn $\zeta+\eta \neq 0$ ist, kann man $H$ so wählen, dass $\zeta(H)+\eta(H) \neq 0$ ist, woraus $(X, Y)=0$ folgt. Damit sind $\mathfrak{g}_{\zeta}$ und $\mathfrak{g}_{\eta}$ zueinander orthogonal.

Die bereits gezeigte Orthogonalität der Cartan-Killing-Form überträgt sich auf die Form $(\cdot, \cdot)_{0}$. Dass bezüglich dieser Form auch $\mathfrak{g}_{\zeta}$ und $\mathfrak{g}_{-\zeta}$ orthogonal sind, ergibt sich sofort aus $\left(\mathfrak{g}_{\zeta}, \mathfrak{g}_{\zeta}\right)=0$.

Aus der Cartan-Killing-Form ergibt sich durch Konjugation der StandardInvolution eine Familie von archimedischen Normen, die in Bijektion zu den maximal kompakten Untergruppen von $G$ steht. Der Raum dieser Normen heißt symmetrischer Raum.

\subsection{Skalarprodukte}

Die aus der Cartan-Killing-Form gewonnene symmetrische Bilinearform $(\cdot, \cdot)_{0}$ liefert in natürlicher Weise eine Norm auf $\mathfrak{g}$.

Gan und Yu konstruieren in ihrer Arbeit GY03 ein Skalarprodukt auf den Oktaven. Im Folgenden soll untersucht werden, inwieweit dieses Skalarprodukt mit dem sich aus der Cartan-Killing-Form ergebenden Skalarprodukt zusammenhängt.

Das Skalarprodukt von Gan und Yu ergibt sich in deren Modell der Oktaven schlicht als Diagonalform; in das in dieser Arbeit verwendete Modell der Oktaven übersetzt es sich ebenfalls in die übliche Diagonalform, nämlich mit 
der Indexmenge $I=\{11,22,12,21,11 \boldsymbol{a}, 22 \boldsymbol{a}, 12 \boldsymbol{a}, 21 \boldsymbol{a}\}$ und der abkürzenden Notation $e_{j k} \boldsymbol{a}:=e_{j k} \boldsymbol{a}$ zu

$$
\left\langle\sum_{i \in I} \xi_{i} e_{i}, \sum_{j \in I} \psi_{j} e_{j}\right\rangle=\sum_{i \in I} \xi_{i} \psi_{i}
$$

Durch die Wahl einer Basis ergibt sich als ein naheliegendes Skalarprodukt für die Gruppe der Endomorphismen eines Vektorraums die Diagonalform über den zugehörigen Elementarmatrizen. Dieses Skalarprodukt überträgt sich unmittelbar auf die zugehörige Lie-Algebra; es sei mit $\langle\cdot, \cdot\rangle_{Y}$ bezeichnet, das zugehörige Norm-Quadrat mit $\tilde{N}(\cdot)$.

Aus der gezeigten Darstellung der Basis der Lie-Algebra (siehe Seite 55) ergibt sich

$$
\begin{array}{ll}
\tilde{N}\left(\underline{U}_{\eta}\right)=6 & \text { für alle kurzen Wurzeln } \eta \in \Phi \text { und } \\
\tilde{N}\left(\underline{U}_{\eta}\right)=2 & \text { für alle langen Wurzeln } \eta \in \Phi .
\end{array}
$$

Das über die Cartan-Killing-Form erhaltene Skalarprodukt ist damit bis auf einen Faktor 4 identisch mit demjenigen, das über das Skalarprodukt auf den Oktaven gewonnen wurde.

\subsection{Renormalisierung}

Um die von Harder und Stuhler gewünschte Konstruktion durchführen zu können, werden die Metriken renormalisiert. In HS03 heißt es im Abschnitt nach 4.8 (eigene Übersetzung - es sei daran erinnert, dass $\left(H_{\zeta}, H_{\zeta}\right)_{0}=$ $\left(H_{\zeta}, H_{\zeta}\right)$ gilt): Die Metrik auf einem Subquotienten $\mathfrak{u}_{\zeta}$, induziert von $(\cdot, \cdot)_{x}$, soll mit dem Faktor $2\left(H_{\zeta}, H_{\zeta}\right)_{0}^{-1}$ multipliziert werden, notiert als $\left.h_{x}\right|_{\mathfrak{u}_{\zeta}}:=$ $\left.2\left(H_{\zeta}, H_{\zeta}\right)_{0}^{-1}(\cdot, \cdot)_{x}\right|_{\mathfrak{u}_{\zeta}}$. Insbesondere erhalten wir mit Bezug auf die StandardBorelgruppe $B_{0}$, die durch die obige Chevalley-Struktur $\left\{X_{\zeta}, H_{\zeta}: \zeta \in \Phi\right\}$ und $\Theta=\Theta_{0}$ gegeben ist: Es ist

$$
h_{0}\left(X_{\zeta}, X_{\zeta}\right)=2 \cdot\left(H_{\zeta}, H_{\zeta}\right)^{-1}\left(X_{\zeta}, X_{\zeta}\right)_{0}=1,
$$

so dass $h_{0}$, eingeschränkt auf $\underline{\mathrm{rad}}_{u}\left(B_{0}\right)$, die Standardform (symmetrisch bilinear bzw. hermitesch) $\perp_{\zeta \in \Phi^{+}}\langle 1\rangle$ ist.

Bemerkung 9.3 Mit Hinsicht auf die auf zwei Arten mögliche Konstruktion des Skalarproduktes stellt sich die Frage, ob die Renormalisierung auch in gewisser Weise auf der Ebene der Oktaven beschrieben werden kann, das heißt, ob das Skalarprodukt für die Oktaven derart verändert werden kann, dass mit einer ähnlichen Zuordnung wie im vorigen Abschnitt beschrieben das renormalisierte Skalarprodukt erhalten wird.

Dies ist tatsächlich möglich, wobei die Frage der genauen Bedeutung dessen hier nicht geklärt werden soll. - Man skaliere die Basiselemente der Oktaven wie folgt: 
- $e_{11}$ und $e_{22}$ mit einem Faktor $r$,

- $e_{12}, e_{11} \boldsymbol{a}$ und $e_{21} \boldsymbol{a}$ mit einem Faktor $s$ und

- $e_{21}, e_{22} \boldsymbol{a}$ und $e_{12} \boldsymbol{a}$ mit einem Faktor $t$.

Zu dieser Umskalierung werde das zuvor in Diagonalform erhaltene Skalarprodukt auf den Endomorphismen derart umskaliert, dass das Skalarprodukt an der Stelle der Elementarmatrix $E_{i j}$ mit den beiden zum i-ten und zum j-ten Basiselement der Oktaven zugehörigen Faktoren multipliziert wird.

Bei Betrachtung der Verteilung der jeweiligen Einträge in den Matrizen der $\underline{U}_{\zeta}$ erkennt man, dass für eine lange Wurzel $\zeta$ je genau ein Eintrag mit $s^{2}$, der andere mit $t^{2}$ skaliert wird. Für kurze Wurzeln $\zeta$ gilt, dass zwei der vier Einträge in den ersten beiden Zeilen oder Spalten mit rs, die anderen beiden mit rt und die beiden Einträge in der rechten unteren $6 \times 6$-Untermatrix mit dem Faktor st skaliert werden. Für $s \neq t$ gilt $s^{2}+t^{2}>2 s t$. Daher kann man $r$, $s$ und $t$ so wählen, dass $s^{2}+t^{2}=2 s t+2 r(s+t)$ ist, sogar so, dass beide Terme gleich 1 sind, zum Beispiel mit den Wahlen $s=0,6$, $t=0,8, r=1 / 70$. Damit ergibt sich mit dem abgewandelten Skalarprodukt $\tilde{N}^{*}\left(\underline{U}_{\zeta}\right)=1$ für alle $\zeta \in \Phi$.

\subsection{Ein Zusammenhang zwischen den Normen und den Ein- bettungen der Hamiltonschen Quaternionen}

Die archimedischen Normen entsprechen eineindeutig den maximal kompakten Untergruppen der Automorphismengruppe $G$ der Oktaven $C$. Es gibt für $G$ noch eine weitere eineindeutige Beschreibung für deren maximal kompakte Untergruppen, wie in diesem Abschnitt gezeigt werden soll.

Satz 9.4 Seien $\mathbb{H}$ die Hamiltonschen Quaternionen, das heißt sei $\mathbb{H}$ die von $i$ und $j$ mit den Relationen $i^{2}=j^{2}=-1$ und $i j=-j i$ erzeugte nicht zerfallende reelle Quaternionenalgebra. Dann gibt es eine kanonische Bijektion

$$
\{\text { Einbettungen } \mathbb{H} \hookrightarrow C\} \quad \rightarrow \quad\{\text { max. kompakte Untergruppen von } G\} \text {. }
$$

Zum Beweis des Satzes reicht es zu zeigen, dass die Stabilisatorgruppe einer Einbettung von $\mathbb{H}$ in $C$ eine kompakte 6-dimensionale Untergruppe von $G$ ist, denn dann ist sie nach dem Satz von Skolem-Noether konjugiert zur standard-maximal-kompakten Untergruppe $K_{0}$ von $G$.

Zunächst ist festzustellen, dass es überhaupt eine Einbettung von $\mathbb{H}$ in $C$ gibt. Dazu betrachte man die in Abschnitt 2.3 eingeführte Konstruktion mittels des Verdopplungsverfahrens. Die Oktaven werden damit aus einer zerfallenden Quaternionenalgebra $D$ konstruiert. $D$ enthält aber immerhin als Unteralgebra $F$ eine Einbettung der komplexen Zahlen, da es eine solche in den reellen $2 \times 2$-Matrizen gibt. Das Verdopplungsverfahren auf $D$ liefert 
auch ein Verdopplungsverfahren auf jeder Unteralgebra, sofern $a \cdot a=\lambda \cdot 1$ in der Unteralgebra liegt, was keine Einschränkung ist. So entsteht aus $F$ eine Quaternionenalgebra; bei geeigneter Wahl des Parameters $\lambda$ entstehen die Hamiltonschen Quaternionen. Dieses $\lambda$ kann aber auch ohne Einschränkung bei der Konstruktion der Oktaven verwendet werden, da die zerfallenden Oktaven bis auf Isomorphie eindeutig sind, und damit ist in $C$ eine Einbettung von $\mathbb{H}$ enthalten.

Es ist auch leicht möglich, eine Einbettung konkret anzugeben, die zudem eine sehr kanonische Form hat. Dazu bilde man die Erzeugenden wie folgt ab:

$$
\begin{array}{rlll}
\varepsilon: \mathbb{H} & \rightarrow & C & \\
1 & \mapsto & 1=\left(\begin{array}{ll}
1 & 0 \\
0 & 1
\end{array}\right)+\left(\begin{array}{ll}
0 & 0 \\
0 & 0
\end{array}\right) \boldsymbol{a}, \\
i & \mapsto & & \left(\begin{array}{rr}
0 & 1 \\
-1 & 0
\end{array}\right)+\left(\begin{array}{ll}
0 & 0 \\
0 & 0
\end{array}\right) \boldsymbol{a}, \\
j & \mapsto & & \left(\begin{array}{ll}
0 & 0 \\
0 & 0
\end{array}\right)+\left(\begin{array}{rr}
1 & 0 \\
0 & -1
\end{array}\right) \boldsymbol{a}, \\
i j & \mapsto & & \left(\begin{array}{ll}
0 & 0 \\
0 & 0
\end{array}\right)+\left(\begin{array}{ll}
0 & 1 \\
1 & 0
\end{array}\right) \boldsymbol{a} .
\end{array}
$$

Man rechnet leicht nach (vgl. die Multiplikationsvorschrift auf Seite 31), dass dies eine Einbettung ist.

Nun ist

$$
\boldsymbol{a}^{\prime}:=\left(\begin{array}{rr}
1 & 0 \\
0 & -1
\end{array}\right)+\left(\begin{array}{ll}
0 & 0 \\
0 & 0
\end{array}\right) \boldsymbol{a} \in \varepsilon(\mathbb{H})^{\perp} .
$$

Nach Bemerkung 2.10 ist damit $C$ als direkte Summe

$$
C \cong \mathbb{H} \oplus \mathbb{H} \boldsymbol{a}^{\prime}
$$

darstellbar. Leicht nachzurechnen ist dabei auch die Gestalt von $\mathbb{H} \boldsymbol{a}^{\prime}$ : Es sind alle Elemente der Form $\left(\begin{array}{r}x-y \\ -y-x\end{array}\right)+\left(\begin{array}{cc}-z & w \\ w & z\end{array}\right)$ mit rellen $x, y, z$ und $w$.

Springer und Veldkamp beschreiben [SV00, Abschnitt 2.1] zu einer aus Verdopplung einer Quaternionenalgebra gewonnenen Oktav-Algebra diejenigen Automorphismen, die die Quaternionenalgebra fixieren: Sie haben in der hier genutzten Notation die Form

$$
x+y \boldsymbol{a}^{\prime} \mapsto u(x)+(p u(y)) \boldsymbol{a}^{\prime} \quad(x, y \in \mathbb{H}),
$$

und dabei ist $u$ ein Automorphismus von $\mathbb{H}$ und $p \in \mathbb{H}$ mit $N(p)=1$. Die Zuordnung der Automorphismen zu den Paaren $(u, p)$ ist eine Bijektion. Nun ist sowohl die Automorphismengruppe von $\mathbb{H}$ eine zur $\mathrm{SO}_{3}$ isomorphe, also dreidimensionale Gruppe als auch die Menge der Quaternionen mit Norm 1 ein dreidimensionaler Raum. Daher ist die Stabilisatorgruppe $G_{\mathbb{H}}$ von $\varepsilon(\mathbb{H})$ in $C$ sechsdimensional. Aus der Kompaktheit von $\mathrm{SO}_{3}$ und der Kompaktheit der Menge der Quaternionen mit Norm 1 folgt die Kompaktheit von $G_{\mathbb{H}}$. 
Weil in $G$ die maximal kompakten Untergruppen die Dimension 6 haben, ist somit $G_{\mathbb{H}}$ eine solche Gruppe.

Man kann die Stabilisatorgruppe sogar von Hand ausrechnen. Dazu nutzt man aus, dass es in der standard-maximal-kompakten Gruppe $K_{0}$ zu jedem Paar $\pm \eta$ von positiver und negativer Wurzel eine zur $\mathrm{SO}_{2}$ isomorphe Untergruppe $\mathrm{SO}_{\eta}$ gibt. Diese kann man konkret bestimmen, wobei nur die wesentlichen Ergebnisse angegeben werden sollen. Es ist

$$
\left(\begin{array}{ll}
1 & a \\
0 & 1
\end{array}\right) \cdot\left(\begin{array}{ll}
1 & 0 \\
b & 1
\end{array}\right) \cdot\left(\begin{array}{ll}
1 & a \\
0 & 1
\end{array}\right)=\left(\begin{array}{cc}
1+a b & 2 a+a^{2} b \\
b & 1+a b
\end{array}\right)
$$

Mit der Wahl

$$
b=\frac{-2 a}{1+a^{2}}
$$

ergibt sich die Matrix

$$
\left(\begin{array}{ll}
\frac{1-a^{2}}{1+a^{2}} & \frac{2 a}{1+a^{2}} \\
\frac{-2 a}{1+a^{2}} & \frac{1-a^{2}}{1+a^{2}}
\end{array}\right),
$$

die im reell zweidimensionalen Raum eine Rechtsdrehung um den Winkel $2 \arctan (a)$ beschreibt.

Nun kann man entsprechend die Produkte

$$
V_{\alpha}(a):=U_{\alpha}(a) \cdot U_{-\alpha}\left(\frac{2 a}{1+a^{2}}\right) \cdot U_{\alpha}(a)
$$

und

$$
V_{\beta}(a):=U_{\beta}(a) \cdot U_{-\beta}\left(\frac{2 a}{1+a^{2}}\right) \cdot U_{\beta}(a)
$$

berechnen und erhält so Elemente aus $K_{0}$. (Der Vorzeichenwechsel beim Argument zur jeweiligen negativen Wurzel erklärt sich aus der negativen Parametrisierung bei diesen Wurzeluntergruppen.) Sie haben die folgende Gestalt - wegen der schöneren Form mit Bezug auf die in Abschnitt 10.1 passend zur Flagge eingeführten Basis $B_{\mathrm{Fl}}$.

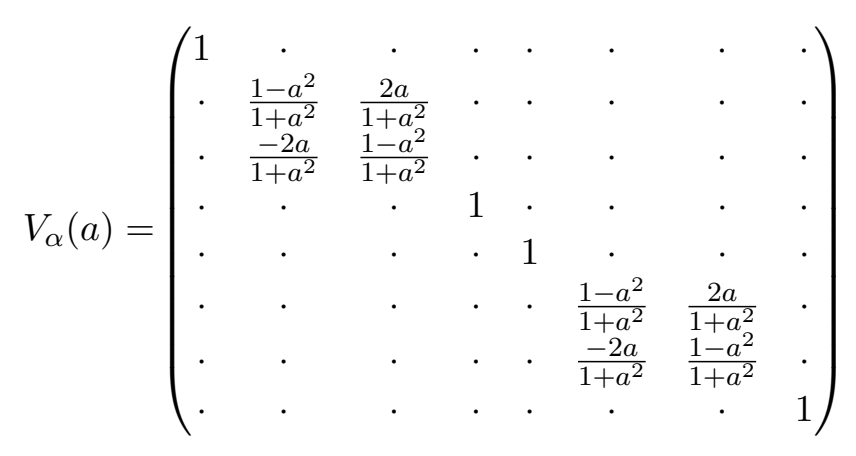


und

$$
V_{\beta}(a)=\left(\begin{array}{cccccccc}
\frac{1-a^{2}}{1+a^{2}} & \frac{2 a}{1+a^{2}} & \cdot & \cdot & \cdot & \cdot & \cdot & \cdot \\
\frac{-2 a}{1+a^{2}} & \frac{1-a^{2}}{1+a^{2}} & \cdot & \cdot & \cdot & \cdot & \cdot & \cdot \\
\cdot & \cdot & \frac{\left(1-a^{2}\right)^{2}}{\left(1+a^{2}\right)^{2}} & \frac{-2 a+2 a^{3}}{\left(1+a^{2}\right)^{2}} & \frac{2 a-2 a^{3}}{\left(1+a^{2}\right)^{2}} & \frac{-4 a^{2}}{\left(1+a^{2}\right)^{2}} & \cdot & \cdot \\
\cdot & \cdot & \frac{2 a-2 a^{3}}{\left(1+a^{2}\right)^{2}} & \frac{\left(1-a^{2}\right)^{2}}{\left(1+a^{2}\right)^{2}} & \frac{4 a^{2}}{\left(1+a^{2}\right)^{2}} & \frac{2 a-2 a^{3}}{\left(1+a^{2}\right)^{2}} & \cdot & \cdot \\
\cdot & \cdot & \frac{-2 a+2 a^{3}}{\left(1+a^{2}\right)^{2}} & \frac{4 a^{2}}{\left(1+a^{2}\right)^{2}} & \frac{\left(1-a^{2}\right)^{2}}{\left(1+a^{2}\right)^{2}} & \frac{-2 a+2 a^{3}}{\left(1+a^{2}\right)^{2}} & \cdot & \cdot \\
\cdot & \cdot & \frac{-4 a^{2}}{\left(1+a^{2}\right)^{2}} & \frac{-2 a+2 a^{3}}{\left(1+a^{2}\right)^{2}} & \frac{2 a-2 a^{3}}{\left(1+a^{2}\right)^{2}} & \frac{\left(1-a^{2}\right)^{2}}{\left(1+a^{2}\right)^{2}} & \cdot & \cdot \\
\cdot & \cdot & \cdot & \cdot & \cdot & \cdot & \frac{1-a^{2}}{1+a^{2}} & \frac{2 a}{1+a^{2}} \\
\cdot & \cdot & \cdot & \cdot & \cdot & \cdot & \frac{-2 a}{1+a^{2}} & \frac{1-a^{2}}{1+a^{2}}
\end{array}\right)
$$

Man kann nachrechnen, dass diese Abbildungen $\varepsilon(\mathbb{H})$ als Menge fixieren; damit müssen sie aber auch $\varepsilon(\mathbb{H})$ als Algebra invariant lassen.

Bemerkung 9.5 Konkret setzt beispielsweise $V_{\beta}(a)$ den inneren Automorphismus von $\mathbb{H}$, der durch die Konjugation mit 1 - ai gegeben wird, fort.

$\{\alpha, \beta\}$ ist eine Wurzelbasis, daher erzeugen $V_{\alpha}$ und $V_{\beta}$ zusammen $K_{0}$. Somit ist der Stabilisator von $\varepsilon(\mathbb{H})$ tatsächlich die standard-maximal-kompakte Untergruppe von $G$, was allerdings wegen der kanonischen Gestalt der Einbettung sicherlich nahelag.

Bemerkung 9.6 Mit der Darstellung 9.4 wird im Übrigen die Angabe einer Parametrisierung der standard-maximal-kompakten Untergruppe erleichtert. 


\section{Weitere Strukturuntersuchungen mit der Bo- relgruppe}

\subsection{Die Filtration zur Standard-Borelgruppe}

$\mathrm{Zu}$ den Oktaven über $\mathbb{R}$ wird hier die Filtrationseigenschaft zur StandardBorelgruppe untersucht.

Die Standard-Borelgruppe wird erzeugt von den beiden folgenden Gruppen $U_{\alpha}$ und $U_{\beta}$ sowie vom Torus

$$
T=\left\{\operatorname{diag}\left(1,1, f, f^{-1}, g, g^{-1}, f g,(f g)^{-1}\right) \mid f, g \in \mathbb{R}^{*}\right\} .
$$

Die Matrizen sind zur Basis $\left\{e_{11}, e_{22}, e_{12}, e_{21}, e_{11} \boldsymbol{a}, e_{22} \boldsymbol{a}, e_{12} \boldsymbol{a}, e_{21} \boldsymbol{a}\right\}$ dargestellt; wie schon vorher stehen Punkte als Matrixeinträge wegen der besseren Lesbarkeit anstelle von Nullen.

$$
\begin{aligned}
& U_{\alpha}=\left\{\left(\begin{array}{cccccccc}
1 & \cdot & \cdot & \cdot & \cdot & \cdot & \cdot & \cdot \\
\cdot & 1 & \cdot & \cdot & \cdot & \cdot & \cdot & \cdot \\
\cdot & \cdot & 1 & \cdot & \cdot & \cdot & \cdot & t \\
\cdot & \cdot & \cdot & 1 & \cdot & \cdot & \cdot & \cdot \\
\cdot & \cdot & \cdot & \cdot & 1 & \cdot & \cdot & \cdot \\
\cdot & \cdot & \cdot & \cdot & \cdot & 1 & \cdot & \cdot \\
\cdot & \cdot & \cdot & t & \cdot & \cdot & 1 & \cdot \\
\cdot & \cdot & \cdot & \cdot & \cdot & \cdot & \cdot & 1
\end{array}\right) \mid t \in \mathbb{R}\right\} \\
& U_{\beta}=\left\{\left(\begin{array}{rrrrrrrr}
1 & \cdot & t & \cdot & \cdot & \cdot & \cdot & \cdot \\
\cdot & 1 & -t & \cdot & \cdot & \cdot & \cdot & \cdot \\
\cdot & \cdot & 1 & \cdot & \cdot & \cdot & \cdot & \cdot \\
-t & t & -t^{2} & 1 & \cdot & \cdot & \cdot & \cdot \\
\cdot & \cdot & \cdot & \cdot & 1 & \cdot & t & \cdot \\
\cdot & \cdot & \cdot & \cdot & \cdot & 1 & \cdot & \cdot \\
\cdot & \cdot & \cdot & \cdot & \cdot & \cdot & 1 & \cdot \\
\cdot & \cdot & \cdot & \cdot & \cdot & t & \cdot & 1
\end{array}\right) \mid t \in \mathbb{R}\right\}
\end{aligned}
$$

Zunächst sei nur das Verhalten unter der Wirkung von $U_{\alpha}$ und $U_{\beta}$ betrachtet. Die Eins der Oktaven $C$, darstellbar als $\mathbf{1}=e_{11}+e_{22}$, wird selbstverständlich von beiden Gruppen fixiert. Gleichzeitig sei sie zunächst aus der Betrachtung herausgenommen, weil sie eine Sonderrolle einnimmt. Das orthogonale Komplement der Eins in der von $e_{11}$ und $e_{22}$ aufgespannten Ebene ist $\left\langle e_{11}-e_{22}\right\rangle$.

Es wird daher nun die Flagge in dem von $\left\{e_{11}-e_{22}, e_{12}, e_{21}, e_{11} \boldsymbol{a}, e_{22} \boldsymbol{a}\right.$, $\left.e_{12} \boldsymbol{a}, e_{21} \boldsymbol{a}\right\}$ aufgespannten Raum $C_{(7)}$ bestimmt; dieser ist gleichzeitig das orthogonale Komplement der Eins in $C$.

Die Gerade $\left\langle e_{11} \boldsymbol{a}\right\rangle$ wird von den beiden Wurzeluntergruppen elementweise fixiert. 
Weitere Fixelemente gibt es nicht (da zum einen $e_{11}-e_{22}$ nicht fixiert wird; und weiter in den Spalten 3, 4 und 6 bis 8 jeweils in einer der beiden Matrizen ein $t$ steht, zu dem es in derselben Zeile kein zweites gibt).

Der einzige zweidimensionale Raum in $C_{(7)}$, der fixiert wird, ist der von $e_{11} \boldsymbol{a}$ und $e_{12} \boldsymbol{a}$ erzeugte Raum, wie man mit analoger Argumentation erkennt.

Im dritten Schritt der Flaggenbildung kommt $e_{21}$ als Erzeuger hinzu. Dann folgt $e_{11}-e_{22}$ und schließlich folgen der Reihe nach noch $e_{12}, e_{21} \boldsymbol{a}$ und $e_{22} \boldsymbol{a}$.

Der Torus $T$ fixiert offenbar alle so gebildeten Räume ebenfalls.

Damit stellt sich eine von der Borelgruppe fixierte Flagge in $C_{(7)}$ wie folgt dar:

$$
\begin{aligned}
\{0\} \subset\left\langle e_{11} \boldsymbol{a}\right\rangle \subset\left\langle e_{11} \boldsymbol{a}, e_{12} \boldsymbol{a}\right\rangle & \subset\left\langle e_{11} \boldsymbol{a}, e_{12} \boldsymbol{a}, e_{21}\right\rangle \subset \\
\left\langle e_{11}-e_{22}, e_{11} \boldsymbol{a}, e_{12} \boldsymbol{a}, e_{21}\right\rangle \subset\left\langle e_{11}-e_{22}, e_{11} \boldsymbol{a}, e_{12} \boldsymbol{a}, e_{21}, e_{12}\right\rangle \subset & \\
& \left\langle e_{11}-e_{22}, e_{11} \boldsymbol{a}, e_{12} \boldsymbol{a}, e_{21}, e_{12}, e_{21} \boldsymbol{a}\right\rangle \subset C .
\end{aligned}
$$

Da der von der Eins aufgespannte Raum fixiert wird und die Flagge in einem direkten Summanden für die Eins gebildet wurde, kann man die Eins an beliebiger Stelle zuordnen und auch noch kleine Veränderungen in dem Raum, zu dem sie zugefügt wird, vornehmen. Man kann auf diese Weise eine von der Borelgruppe fixierte Flagge in $C$ erhalten, die direkt zur Basis zum Standardtorus passt:

$$
\begin{aligned}
& \{0\} \subset\left\langle e_{11} \boldsymbol{a}\right\rangle \subset\left\langle e_{11} \boldsymbol{a}, e_{12} \boldsymbol{a}\right\rangle \subset\left\langle e_{11} \boldsymbol{a}, e_{12} \boldsymbol{a}, e_{21}\right\rangle \subset \\
& \left\langle e_{11} \boldsymbol{a}, e_{12} \boldsymbol{a}, e_{21}, e_{11}\right\rangle \subset\left\langle e_{11} \boldsymbol{a}, e_{12} \boldsymbol{a}, e_{21}, e_{11}, e_{22}\right\rangle \subset \\
& \left\langle e_{11} \boldsymbol{a}, e_{12} \boldsymbol{a}, e_{21}, e_{11}, e_{22}, e_{12}\right\rangle \subset \\
& \left\langle e_{11} \boldsymbol{a}, e_{12} \boldsymbol{a}, e_{21}, e_{11}, e_{22}, e_{12}, e_{21} \boldsymbol{a}\right\rangle \subset C .
\end{aligned}
$$

Diese Flagge hat die hübsche Eigenschaft, dass jeweils der $k$-dimensionale Raum das orthogonale Komplement des $(8-k)$-dimensionalen Raumes ist. Sie sei als Standardflagge bezeichnet.

Bezüglich der eben bestimmten Flagge hat die Borelgruppe nun Dreiecksgestalt. Als Basis für die weiteren Betrachtungen in diesem Kapitel wird daher definiert:

$$
B_{\mathrm{Fl}}:=\left\{e_{11} \boldsymbol{a}, e_{12} \boldsymbol{a}, e_{21}, e_{11}, e_{22}, e_{12}, e_{21} \boldsymbol{a}, e_{22} \boldsymbol{a}\right\}
$$


Die Matrizen von $U_{\alpha}$ haben dann die Form

$$
\left(\begin{array}{cccccccc}
1 & \cdot & \cdot & \cdot & \cdot & \cdot & \cdot & \cdot \\
\cdot & 1 & t & \cdot & \cdot & \cdot & \cdot & \cdot \\
\cdot & \cdot & 1 & \cdot & \cdot & \cdot & \cdot & \cdot \\
\cdot & \cdot & \cdot & 1 & \cdot & \cdot & \cdot & \cdot \\
\cdot & \cdot & \cdot & \cdot & 1 & \cdot & \cdot & \cdot \\
\cdot & \cdot & \cdot & \cdot & \cdot & 1 & t & \cdot \\
\cdot & \cdot & \cdot & \cdot & \cdot & \cdot & 1 & \cdot \\
\cdot & \cdot & \cdot & \cdot & \cdot & \cdot & \cdot & 1
\end{array}\right)
$$

und die von $U_{\beta}$ die Form

$$
\left(\begin{array}{rrrrrrrr}
1 & t & \cdot & \cdot & \cdot & \cdot & \cdot & \cdot \\
\cdot & 1 & \cdot & \cdot & \cdot & \cdot & \cdot & \cdot \\
\cdot & \cdot & 1 & -t & t & -t^{2} & \cdot & \cdot \\
\cdot & \cdot & \cdot & 1 & \cdot & t & \cdot & \cdot \\
\cdot & \cdot & \cdot & \cdot & 1 & -t & \cdot & \cdot \\
\cdot & \cdot & \cdot & \cdot & \cdot & 1 & \cdot & \cdot \\
\cdot & \cdot & \cdot & \cdot & \cdot & \cdot & 1 & t \\
\cdot & \cdot & \cdot & \cdot & \cdot & \cdot & \cdot & 1
\end{array}\right) .
$$

Die Gesamtheit aller Matrizen der $U_{\eta}$ mit positiver Wurzel lässt sich platzsparend in einer oberen Dreiecksmatrix darstellen: Dabei steht $[\eta]$ für die Stelle, an der in der Matrix $U_{\eta}(t)$ der Parameter $t$ steht; ein etwaiges Minuszeichen ist davorgesetzt. Bei doppelten eckigen Klammern steht das Quadrat von $t$. Die so beschreibende Matrix ist

$$
\left(\begin{array}{cccccccc}
1 & {[\beta]} & -[\alpha+\beta] & {[\alpha+2 \beta]} & -[\alpha+2 \beta] & {[\alpha+3 \beta]} & {[2 \alpha+3 \beta]} & -[[\alpha+2 \beta]] \\
. & 1 & {[\alpha]} & -[\alpha+\beta] & {[\alpha+\beta]} & -[\alpha+2 \beta] & [\alpha+\beta]] & {[2 \alpha+3 \beta]} \\
. & . & 1 & -[\beta] & {[\beta]} & -[[\beta]] & -[\alpha+2 \beta] & -[\alpha+3 \beta] \\
. & . & . & 1 & . & {[\beta]} & -[\alpha+\beta] & -[\alpha+2 \beta] \\
. & . & . & . & 1 & -[\beta] & {[\alpha+\beta]} & {[\alpha+2 \beta]} \\
. & . & . & . & . & 1 & {[\alpha]} & {[\alpha+\beta]} \\
. & . & . & . & . & . & 1 & {[\beta]} \\
. & . & . & . & . & . & . & 1
\end{array}\right)
$$

Der Torus in der Parametrisierung durch $f$ und $g$, der vorher die Form $T=\operatorname{diag}\left(1,1, f, f^{-1}, g, g^{-1}, f g,(f g)^{-1}\right)$ hatte, hat zur neuen Basis die Form

$$
T_{\mathrm{Fl}}:=\operatorname{diag}\left(g, f g, f^{-1}, 1,1, f,(f g)^{-1}, g^{-1}\right) .
$$

\subsection{Invariante Unterräume}

Betrachtet man die Multiplikationsvorschrift der Oktaven

$$
\left(\left(\begin{array}{ll}
a & b \\
c & d
\end{array}\right),\left(\begin{array}{ll}
e & f \\
g & h
\end{array}\right)\right) \cdot\left(\left(\begin{array}{cc}
j & k \\
l & m
\end{array}\right),\left(\begin{array}{ll}
n & o \\
p & q
\end{array}\right)\right)=
$$




$$
\begin{aligned}
&\left(\left(\begin{array}{rr}
a j+b l+e q-g o & a k+b m+f q-h o \\
c j+d l-e p+g n & c k+d m-f p+h n
\end{array}\right),\right. \\
&\left.\left(\begin{array}{ll}
a n+c o+e m-f l & b n+d o-e k+f j \\
a p+c q+g m-h l & b p+d q-g k+h j
\end{array}\right)\right),
\end{aligned}
$$

so erkennt man:

- Der eindimensionale invariante Unterraum der Standardflagge ist sogar eine Unteralgebra; dabei ist jedes Produkt null.

- Ebenso ist der zweidimensionale Raum der Standardflagge eine Unteralgebra mit trivialer Multiplikation.

- Der dreidimensionale Raum ist ebenfalls eine Unteralgebra, in der jedoch die Multiplikation nicht mehr trivial ist. Die Einschränkung der Multiplikationsvorschrift ergibt:

$$
\begin{aligned}
\left(\left(\begin{array}{ll}
\cdot & \cdot \\
c & \cdot
\end{array}\right),\left(\begin{array}{ll}
e & f \\
\cdot & \cdot
\end{array}\right)\right) \cdot\left(\left(\begin{array}{ll}
\cdot & \cdot \\
l & \cdot
\end{array}\right),\left(\begin{array}{ll}
n & o \\
\cdot & \cdot
\end{array}\right)\right) & \\
& \left(\left(\begin{array}{ll}
\cdot & \cdot \\
\cdot & \cdot
\end{array}\right),\left(\begin{array}{ccc}
c o-f l & \cdot \\
\cdot & \cdot
\end{array}\right)\right) .
\end{aligned}
$$

Genauso ergeben die vier- bis sechsdimensionalen Unterräume Unteralgebren, wie man an den jeweiligen Einschränkungen der Multiplikationsvorschrift sieht:

- Die Einschränkung für den vierdimensionalen Raum ist:

$$
\begin{aligned}
\left(\left(\begin{array}{ll}
a & \cdot \\
c & \cdot
\end{array}\right),\left(\begin{array}{ll}
e & f \\
\cdot & \cdot
\end{array}\right)\right) \cdot\left(\left(\begin{array}{ll}
j & \cdot \\
l & \cdot
\end{array}\right)\right. & \left.\left(\begin{array}{ll}
n & o \\
\cdot & \cdot
\end{array}\right)\right)= \\
& \left(\left(\begin{array}{ll}
a j & \cdot \\
c j & \cdot
\end{array}\right),\left(\begin{array}{cc}
a n+c o-f l & f j \\
\cdot & \cdot
\end{array}\right)\right),
\end{aligned}
$$

- für den fünfdimensionalen Raum:

$$
\begin{aligned}
\left(\left(\begin{array}{ll}
a & \cdot \\
c & d
\end{array}\right),\left(\begin{array}{ll}
e & f \\
\cdot & \cdot
\end{array}\right)\right) \cdot\left(\left(\begin{array}{ll}
j & \cdot \\
l & m
\end{array}\right),\left(\begin{array}{cc}
n & o \\
\cdot & \cdot
\end{array}\right)\right)= \\
\left(\left(\begin{array}{cc}
a j & \cdot \\
c j+d l & d m
\end{array}\right),\left(\begin{array}{cc}
a n+c o+e m-f l & d o+f j \\
\cdot & \cdot
\end{array}\right)\right),
\end{aligned}
$$

- und für den sechsdimensionalen:

$$
\left(\left(\begin{array}{ll}
a & b \\
c & d
\end{array}\right),\left(\begin{array}{ll}
e & f \\
\cdot & \cdot
\end{array}\right)\right) \cdot\left(\left(\begin{array}{cc}
j & k \\
l & m
\end{array}\right),\left(\begin{array}{ll}
n & o \\
\cdot & \cdot
\end{array}\right)\right)=
$$




$$
\begin{aligned}
&\left(\left(\begin{array}{cc}
a j+b l & a k+b m \\
c j+d l & c k+d m
\end{array}\right)\right. \\
&\left.\left(\begin{array}{cc}
a n+c o+e m-f l & b n+d o-e k+f j \\
\cdot & \cdot
\end{array}\right)\right) .
\end{aligned}
$$

- Der siebendimensionale Raum ergibt keine Unteralgebra.

Damit ist im Speziellen der dreidimensionale Raum isomorph zum Raum der Matrizen der Form

$$
\left(\begin{array}{rrrr}
\cdot & c & f & e \\
\cdot & \cdot & \cdot & f \\
\cdot & \cdot & \cdot & -c \\
\cdot & \cdot & \cdot & \cdot
\end{array}\right)
$$

und somit eine assoziative, nicht kommutative Algebra. Jedes Produkt aus drei oder mehr Faktoren ergibt null.

Es wäre durch Hinzunahme der Eins noch möglich, diese Algebra zu einer vierdimensionalen assoziativen Algebra zu erweitern. Der vierdimensionale Raum in der Standardflagge ist hingegen nicht mehr assoziativ: im Eintrag zu $e_{11} \boldsymbol{a}$ - und nur dort - treten Veränderungen beim Umklammern auf.

\subsection{Versuch einer Deutung}

Bezüglich der neuen Basis $B_{\mathrm{Fl}}=\left\{e_{11} \boldsymbol{a}, e_{12} \boldsymbol{a}, e_{21}, e_{11}, e_{22}, e_{12}, e_{21} \boldsymbol{a}, e_{22} \boldsymbol{a}\right\}$ haben die benannten Erzeuger (Repräsentanten) $\sigma$ und $\tau$ der Weylgruppe die Form

$$
\sigma_{B_{\mathrm{Fl}}}=\left(\begin{array}{rrrrrrrr}
\cdot & \cdot & 1 & \cdot & \cdot & \cdot & \cdot & \cdot \\
1 & \cdot & \cdot & \cdot & \cdot & \cdot & \cdot & \cdot \\
\cdot & \cdot & \cdot & \cdot & \cdot & \cdot & 1 & \cdot \\
\cdot & \cdot & \cdot & \cdot & 1 & \cdot & \cdot & \cdot \\
\cdot & \cdot & \cdot & 1 & \cdot & \cdot & \cdot & \cdot \\
\cdot & -1 & \cdot & \cdot & \cdot & \cdot & \cdot & \cdot \\
\cdot & \cdot & \cdot & \cdot & \cdot & \cdot & \cdot & -1 \\
\cdot & \cdot & \cdot & \cdot & \cdot & 1 & \cdot & \cdot
\end{array}\right)
$$

und

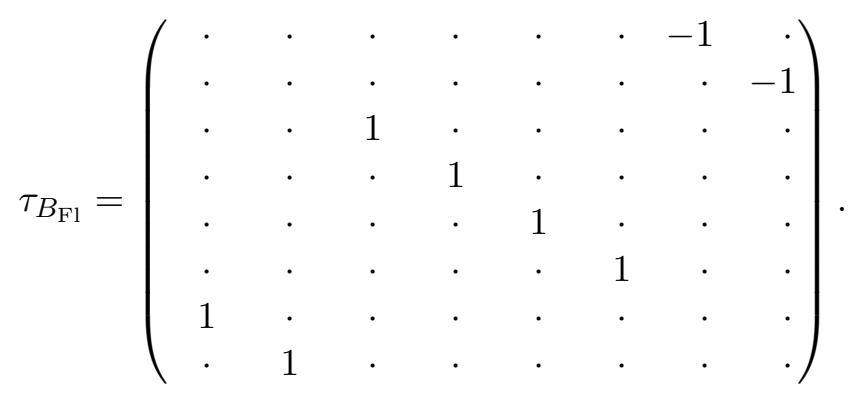


Abkürzend soll dies in Erweiterung der Notation von Permutationen um die Darstellung von Vorzeichenwechseln als $\sigma=(12-68-73)(45)$ und $\tau=(17-1)(28-2)$ notiert werden.

Da $\sigma$ eine Permutation der sechs „entscheidenden“ (also in der alten Basis: dritter bis achter Basisvektor) Basisvektoren ist, gibt es zu jedem der sechs Basisvektoren eine Borelgruppe, bezüglich der dieser Vektor ein Fixelement ist. (Natürlich sind es je genau zwei Borelgruppen, wenn man noch $\tau$, also die gesamte Weylgruppe mit betrachtet.)

Es ist folglich kein Eintrag in den Matrizen bei den Oktaven ausgezeichnet, abgesehen von den beiden die Eins formenden Einträgen.

Beispielhaft sei die Borelgruppe bzw. seien die Gruppen $U_{\eta}$ einmal mit $\sigma$ konjugiert. Dies ergibt als Übersichtsdarstellung:

$$
\left(\begin{array}{cccccccc}
1 & \cdot & -[\alpha+\beta] & {[\alpha+2 \beta]} & -[\alpha+2 \beta] & . & {[2 \alpha+3 \beta]} & -[[\alpha+2 \beta]] \\
-[-\beta] & 1 & {[\alpha]} & -[\alpha+\beta] & {[\alpha+\beta]} & -[\alpha+2 \beta] & {[[\alpha+\beta]]} & {[2 \alpha+3 \beta]} \\
\cdot & \cdot & 1 & \cdot & \cdot & \cdot & -[\alpha+2 \beta] & \cdot \\
\cdot & \cdot & {[-\beta]} & 1 & \cdot & \cdot & -[\alpha+\beta] & -[\alpha+2 \beta] \\
\cdot & \cdot & -[-\beta] & \cdot & 1 & \cdot & {[\alpha+\beta]} & {[\alpha+2 \beta]} \\
-[-\alpha-3 \beta] & \cdot & -[[-\beta]] & -[-\beta] & {[-\beta]} & 1 & {[\alpha]} & {[\alpha+\beta]} \\
\cdot & \cdot & \cdot & \cdot & \cdot & \cdot & 1 & \cdot \\
\cdot & \cdot & {[-\alpha-3 \beta]} & \cdot & \cdot & \cdot & -[-\beta] & 1
\end{array}\right)
$$

(Es sind entfallen: $U_{\beta}$ und $U_{\alpha+3 \beta}$, dafür sind $U_{-\beta}$ und $U_{-\alpha-3 \beta}$ hinzugekommen.)

Zur umgeordneten Basis $B^{\prime}:=\left\{e_{12} \boldsymbol{a}, e_{12}, e_{11} \boldsymbol{a}, e_{22}, e_{11}, e_{22} \boldsymbol{a}, e_{21}, e_{21} \boldsymbol{a}\right\}$ - diese Umsortierung entspricht bis auf Vorzeichen genau der Umkehrung der Konjugation mit $\sigma$ - ergibt sich wieder eine obere Dreiecksform:

$$
\left(\begin{array}{cccccccc}
1 & -[\alpha+2 \beta] & -[-\beta] & {[\alpha+\beta]} & -[\alpha+\beta] & {[2 \alpha+3 \beta]} & {[\alpha]} & {[[\alpha+\beta]]} \\
\cdot & 1 & -[-\alpha-3 \beta] & {[-\beta]} & -[-\beta] & {[\alpha+\beta]} & -[[-\beta]] & {[\alpha]} \\
\cdot & . & . & -[\alpha+2 \beta] & {[\alpha+2 \beta]} & -[[\alpha+2 \beta]] & -[\alpha+\beta] & {[2 \alpha+3 \beta]} \\
\cdot & . & . & 1 & . & {[\alpha+2 \beta]} & -[-\beta] & {[\alpha+\beta]} \\
\cdot & . & . & . & 1 & -[\alpha+2 \beta] & {[-\beta]} & -[\alpha+\beta] \\
\cdot & . & . & . & . & 1 & {[-\alpha-3 \beta]} & -[-\beta] \\
\cdot & . & . & . & . & . & 1 & -[\alpha+2 \beta] \\
\cdot & . & . & . & . & . & . & 1
\end{array}\right)
$$

Führte man die Permutation mit Vorzeichenwechseln aus, ergäbe sich zum Vergleich die folgende Gestalt. Das Muster der Vorzeichen ist dann automatisch dasselbe wie bei den Wurzeluntergruppen bezüglich der kanonischen Basis, dies aber um den Preis von Vorzeichen in der veränderten Basis.

$$
\left(\begin{array}{cccccccc}
1 & {[\alpha+2 \beta]} & -[-\beta] & {[\alpha+\beta]} & -[\alpha+\beta] & {[2 \alpha+3 \beta]} & {[\alpha]} & -[[\alpha+\beta]] \\
\cdot & 1 & {[-\alpha-3 \beta]} & -[-\beta] & {[-\beta]} & -[\alpha+\beta] & {[[-\beta]]} & {[\alpha]} \\
\cdot & . & 1 & -[\alpha+2 \beta] & {[\alpha+2 \beta]} & -[[\alpha+2 \beta]] & -[\alpha+\beta] & -[2 \alpha+3 \beta] \\
\cdot & . & . & 1 & . & {[\alpha+2 \beta]} & -[-\beta] & -[\alpha+\beta] \\
. & . & . & . & 1 & -[\alpha+2 \beta] & {[-\beta]} & {[\alpha+\beta]} \\
. & . & . & . & . & 1 & {[-\alpha-3 \beta]} & {[-\beta]} \\
. & . & . & . & . & . & 1 & {[\alpha+2 \beta]} \\
. & . & . & . & . & . & . & 1
\end{array}\right)
$$

Die Einträge in einer Flagge (egal auf welche der errechneten bezogen) sind 
tatsächlich in einer gewissen Reihenfolge geordnet. Zur Beschreibung dieser Ordnung beachte man zunächst, dass man den Basisvektoren über den Torus auf kanonische Weise eine Wurzel zuordnen kann: Jeder Basisvektor gehört vermöge seiner Position zu einem Eintrag im Torus; dieser Eintrag steht gleichzeitig für einen Charakter, der wiederum einer Wurzel zugeordnet ist. Identifiziert man so die Basisvektoren mit Wurzeln, wobei $e_{11}$ und $e_{22}$ keiner Wurzel beziehungsweise dem Nullvektor im Wurzelraum zugeordnet werden, so sind die Basiselemente in ihrer Reihenfolge des Auftretens in der Flagge genau absteigend nach ihrer Höhe als Wurzel geordnet.

Sie gehören der Reihe nach zu den Wurzeln

$$
\alpha+2 \beta, \alpha+\beta, \beta,(0,)(0,)-\beta,-\alpha-\beta \text { und }-\alpha-2 \beta .
$$

Wohlgemerkt sind dies sämtliche kurze Wurzeln.

Leicht zu verifizieren ist dann die Beobachtung, dass die Basisvektoren außer $e_{11}$ und $e_{22}$ - ebenso in der Reihenfolge für die Flagge durchlaufen werden, wenn man, beginnend bei der Wurzel größter Höhe, abwechselnd mit den Spiegelungen $s_{\beta}$ und $s_{\alpha}$ operiert.

\subsection{Algebraische Beschreibung der Flagge}

Satz 10.1 Eine von einer Borelgruppe fixierte Flagge in der Form 10.1) (siehe Seite 67) ist eineindeutig durch Angabe ihrer ersten beiden Räume gegeben. Zudem können diese ersten beiden Räume eineindeutig auf die folgende Art charakterisiert werden:

1. Man wähle einen eindimensionalen Unterraum $V$ in der Oktavalgebra, für den $V \cdot V=\{0\}$ gilt.

2. Man wähle einen zweidimensionalen Unterraum $W \supset V$ in der Oktavalgebra, für den $W \cdot W=\{0\}$ gilt.

Beweis. Leicht nachzuprüfen ist, dass der von $e_{11} \boldsymbol{a}$ und $e_{12} \boldsymbol{a}$ aufgespannte Raum nur triviale Produkte hat. Damit erfüllt die Flagge (10.1) die genannte Beschreibung, und es reicht für die Charakterisierung zu zeigen, dass jede Wahl von Räumen wie im Satz mit einem Oktaven-Isomorphismus in die Form (10.1) überführt werden kann.

Das Quadrat eines Oktaven-Elements $x=(y+z \boldsymbol{a})$ berechnet sich nach 2.2 ) wie folgt:

$$
x^{2}=(y+z \boldsymbol{a})^{2}=\operatorname{Spur}(y) \cdot x-N(x) \cdot \mathbf{1} .
$$

Im Folgenden seien Koordinatendarstellungen von Elementen der Oktaven weiterhin immer bezüglich der (Standardflaggen-)Basis

$$
B_{\mathrm{Fl}}=\left\{e_{11} \boldsymbol{a}, e_{12} \boldsymbol{a}, e_{21}, e_{11}, e_{22}, e_{12}, e_{21} \boldsymbol{a}, e_{22} \boldsymbol{a}\right\}
$$


gegeben.

Sei $V$ von $v=\left(v_{1}, \ldots, v_{8}\right)^{t}$ aufgespannt. Anhand der Rechenregeln ist sofort zu erkennen, dass in dem von $e_{11}$ und $e_{22}$ aufgespannten Raum kein Quadrat verschwindet. Daher darf, ggf. nach Operation mit einer geeigneten Potenz des Weylgruppenelements $\bar{\sigma}$ (das im Wesentlichen eine Permutation der anderen sechs Basiselemente darstellt) angenommen werden, dass $v_{1} \neq 0$ gilt, und damit auch, dass $v_{1}=1$ gilt.

Aus der Voraussetzung $v^{2}=0$ ergibt sich nun mit 10.2), dass die Spur der ersten Matrix in der üblichen Darstellung von $v$ verschwinden und somit $v_{4}+v_{5}=0$ sein muss. Weiter kann man aus 10.2 nur die ohnehin nötige Bedingung $0=N(v)=v_{4} v_{5}-v_{3} v_{6}-v_{1} v_{8}+v_{2} v_{7}$ folgern.

Damit ist aber zu erkennen, dass $v$ in der Bahn von $e_{11} \boldsymbol{a}$ unter der $\mathrm{Au}$ tomorphismengruppe liegt, denn durch Multiplikation von Elementen der Wurzeluntergruppen zu den Wurzeln $-\beta,-\alpha-\beta,-\alpha-2 \beta,-\alpha-3 \beta$ und $-2 \alpha-3 \beta$ lässt sich ein Automorphismus generieren, bei dem in der Matrixdarstellung in der ersten Spalte der zweite, dritte, vierte, sechste und siebte Eintrag frei wählbar sind (nämlich direkt über die Parameter in den Wurzeluntergruppen). Der fünfte Eintrag ist durch die Spurbedingung festgelegt und der achte Eintrag ohnehin durch die Bedingung, dass die Norm des Bildes von $e_{11} \boldsymbol{a}$ verschwinden muss.

Hierzu das Ergebnis der konkreten Rechnung zur Verdeutlichung:

$$
U_{-\beta}(p) \cdot U_{-\alpha-\beta}(q) \cdot U_{-\alpha-2 \beta}(r) \cdot U_{-\alpha-3 \beta}(s) \cdot U_{-2 \alpha-3 \beta}(t)=
$$

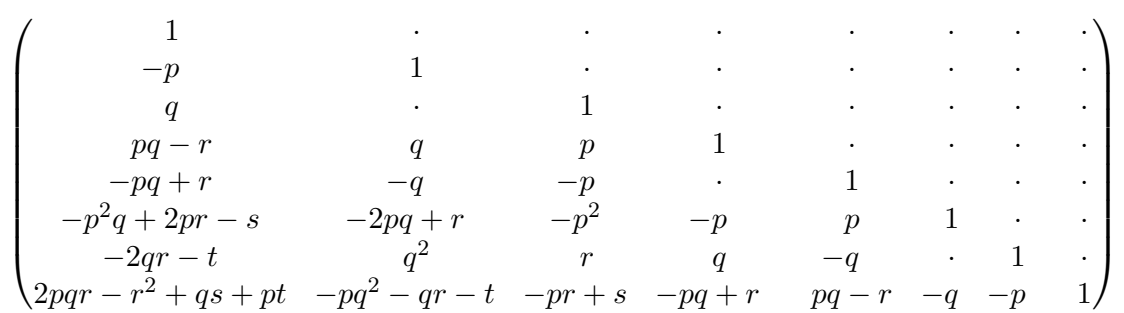

Die Rechnung ist im Anhang A.3 ausgeführt.

Damit kann ohne Einschränkung angenommen werden, dass $v=e_{11} \boldsymbol{a}$ ist. Aus der Multiplikationsvorschrift ist zu erkennen, dass die Menge aller Oktavenelemente, deren Produkt mit $e_{11} \boldsymbol{a}$ zu beiden Seiten verschwindet, der von $e_{11} \boldsymbol{a}, e_{12} \boldsymbol{a}$ und $e_{21}$ aufgespannte Raum ist.

Außerdem ist $\sigma \tau \sigma^{-1}=(23-2)(67-6)$ (vgl. Seite 71). Daher kann ggf. nach Operation mit $\sigma \tau \sigma^{-1}$ angenommen werden, dass es in $W$ ein Element $w=\left(w_{1}, \ldots, w_{8}\right)^{t}$ mit $w_{2}=1$ gibt. Dann ist es möglich, $w$ aus $e_{12} \boldsymbol{a}$ durch Multiplikation mit einem geeigneten Produkt aus Elementen der Wurzeluntergruppen $\mathrm{zu}-\alpha$ und $\beta$ ähnlich wie oben zu erzeugen. Damit ist die Eindeutigkeit der Charakterisierung der beiden ersten Räume gezeigt. 
Es bleibt zu zeigen, dass die restlichen Räume der Flagge bereits durch die ersten beiden eindeutig bestimmt sind. Dies geht am elegantesten auf algebraischem Wege.

Der dreidimensionale Raum $W_{3}$ der Flagge ist, wie schon indirekt oben angemerkt, genau der Raum aller Oktavenelemente, deren Produkt mit dem eindimensionalen Raum $V$ zu beiden Seiten verschwindet.

Der vierdimensionale Raum der Flagge lässt sich eindeutig beschreiben als der Raum aller Oktavenelemente $z$ mit $V \cdot z=\{0\}$.

Der fünfdimensionale Raum $W_{5}$ ist der Raum aller Oktavenelemente $z$ mit $z \cdot V \subseteq V$ bzw. äquivalent $V \cdot z \subseteq V$.

Der sechsdimensionale Raum ist der Raum aller Oktavenelemente $z$ mit $z \cdot W \subseteq W$ bzw. äquivalent $W \cdot z \subseteq W$.

Der siebendimensionale Raum der Flagge ist schließlich der Raum aller Oktavenelemente $z$ mit $z \cdot W_{5} \subseteq W_{5}$ bzw. äquivalent $W \cdot z \subseteq W_{5}$ bzw. äquivalent $W_{3} \cdot z \subseteq W_{5}$.

Alle diese Beschreibungen lassen sich leicht anhand der Multiplikationstabelle in der Form auf Seite 32 verifizieren.

Damit ist der Satz bewiesen.

Bemerkung 10.2 Dass bei der Beschreibung des vierdimensionalen Raumes eine asymmetrische Bedingung verwendet wurde, liegt an der Dualität von $e_{11}$ und $e_{22}$ : Genau bei den Basiselementen ungleich $e_{11}$ und $e_{22}$, zu denen $e_{11}$ eine Rechtseins ist, ist $e_{22}$ eine Linkseins und umgekehrt. $e_{11}$ und $e_{22}$ sind als Erzeuger bezüglich der Standardbasis in der Flagge problemlos vertauschbar. Wählt man das umgekehrte Produkt in der Bedingung für den vierdimensionalen Raum, also $z \cdot V=\{0\}$, so erhält man $e_{22}$ als vierten Erzeuger anstelle von $e_{11}$.

Bemerkung 10.3 Die fünf-bis siebendimensionalen Räume der Flagge besitzen eine weitere eindeutige Charakterisierung. Sie sind die zum drei- bis eindimensionalen Raum orthogonalen Räume. Dies ergibt sich aus der AntiDiagonalgestalt der Bilinearform, wie man leicht nachrechnet. Zudem ist der vierdimensionale Raum zu sich selbst orthogonal.

\subsection{Zerfällung des Torus}

Ähnlich wie bei der algebraischen Beschreibung der Flagge kann man einen zerfallenden maximalen Torus über algebraische Bedingungen beschreiben. Als ersten Raum in der Zerfällung nehme man einen (eindimensionalen) Raum $\tilde{V}$ mit $\tilde{V} \cdot \tilde{V}=\{0\}$. Wie schon oben gezeigt, lässt sich jeder solche Raum per Automorphismus in den Raum $V_{1}:=V=\left\langle e_{11} \boldsymbol{a}\right\rangle$ aus der Standardflagge überführen.

Als zweiten Raum wähle man einen Raum $\tilde{V}_{2}$ mit $\tilde{V}_{2} \cdot V_{1}=V_{1} \cdot \tilde{V}_{2}=\{0\}$. Aus dem Beweis oben folgt, dass $\tilde{V}_{2}$ von einem Vektor $v$ aus $W_{3}$ erzeugt 
werden muss und dass dieser mit einem Automorphismus in $e_{12} \boldsymbol{a}$ überführt werden kann. Man kann daher $V_{2}=\left\langle e_{12} \boldsymbol{a}\right\rangle$ annehmen.

Als dritten Raum wähle man einen weiteren Raum $\tilde{V}_{3}$ mit $\tilde{V}_{3} \cdot V_{1}=V_{1} \cdot \tilde{V}_{3}=$ $\{0\}$, der nicht in dem von $V_{1}$ und $V_{2}$ aufgespannten Raum liegt. (Alternativ zur letzten Bedingung könnte man $\tilde{V}_{3} \cdot V_{2}=V_{1}$ fordern.) In gleicher Weise wie oben lässt sich dieser Raum mit Hilfe der Wurzeluntergruppen zu $\alpha$ und $\alpha+\beta$ in $\left\langle e_{21}\right\rangle=V_{3}$ überführen.

Als vierten Raum wähle man einen von $V_{1}$ verschiedenen Raum $\tilde{V}_{4}$ mit $\tilde{V}_{4}$. $V_{2}=\{0\}$ und $\tilde{V}_{4} \cdot V_{3}=\{0\}$. (Statt $\tilde{V}_{4} \neq V_{1}$ zu fordern, könnte man auch $\tilde{V}_{4}$. $V_{1}=V_{1}$ oder $\tilde{V}_{4} \cdot \tilde{V}_{4}=\tilde{V}_{4}$ als Bedingung stellen.) Dies bestimmt $\tilde{V}_{4}$ eindeutig bis auf einen $V_{1}$-Anteil. Durch Operation mit der Wurzeluntergruppe zu $\alpha+2 \beta$ kann auch dieser Raum in $V_{4}=\left\langle e_{11}\right\rangle$ überführt werden, ohne dass sich - wie vorher auch entsprechend - an $V_{1}$ bis $V_{3}$ etwas ändert.

Als fünften Raum wähle man $V_{5}$ mit $V_{5} \cdot V_{4}=V_{4} \cdot V_{5}=\{0\}$. (Dadurch ist $V_{5}$ bereits eindeutig als $\left\langle e_{22}\right\rangle$ bestimmt.)

Als sechsten Raum wähle man $\tilde{V}_{6}$ mit $\tilde{V}_{6} \cdot V_{2}=\{0\}$ und $\tilde{V}_{6} \cdot V_{4}=\{0\}$. Dies bestimmt wiederum $\tilde{V}_{6}$ nur eindeutig bis auf einen $V_{1}$-Anteil. Durch Operation mit der Wurzeluntergruppe zu $\alpha+3 \beta$ kann dieser Raum ohne Veränderung der anderen Räume in $V_{6}=\left\langle e_{12}\right\rangle$ überführt werden.

Der siebte Raum $\tilde{V}_{7}$ ist bis auf einen $V_{1}$-Anteil eindeutig durch die Bedingungen $\tilde{V}_{7} \cdot V_{3}=\{0\}$ und $\tilde{V}_{7} \cdot V_{4}=\{0\}$ bestimmt. Hier führt eine Operation mit der Wurzeluntergruppe zu $2 \alpha+3 \beta$ zum kanonischen Raum $V_{7}=\left\langle e_{21} \boldsymbol{a}\right\rangle$. Der achte Raum $V_{8}=\left\langle e_{22} \boldsymbol{a}\right\rangle$ schließlich ist beispielsweise durch die Bedingungen $V_{8} \cdot V_{6}=\{0\}$ und $V_{8} \cdot V_{3}=V_{7}$ eindeutig bestimmt.

Damit ist gezeigt, dass in jedem Fall eine gleichgeartete, vollständige Zerfällung des Torus vorgenommen werden kann.

Bemerkung 10.4 Anders als es der Aufbau der Flagge vermuten lässt, ist es nicht möglich, den vierten Raum in der Zerfällung ohne Bezug auf den zweiten zu definieren, denn in dem von $e_{11}, e_{21}$ und $e_{11}$ a aufgespannten Raum lassen sich $e_{11}$ und zum Beispiel $e_{11}+e_{21}$ bezüglich ihres Verhaltens bei der Multiplikation nicht voneinander unterscheiden, und es gibt auch keinen Automorphismus, der einen $e_{21}$-Anteil entfernen kann, ohne einen vorher festgelegten Raum zu verändern. Analoges gilt auch für die mögliche Alternative $e_{22}$ und den von $e_{22}, e_{21}$ und $e_{11}$ a aufgespannten Raum. 


\section{Das Komplementärpolyeder für Bewertungen aus den Standardapartments}

Als Abschluss dieser Arbeit soll das Komplementärpolyeder zur Automorphismengruppe $G$ der zerfallenden Oktaven $C$ über $\mathbb{R}$ für die Fälle beleuchtet werden, in denen sämtliche Normen zum jeweiligen Standardapartment gehören.

Es sei $B$ eine Borelgruppe, $\left\{\alpha_{1}, \ldots, \alpha_{n}\right\}$ eine dazu passende Wurzelbasis und $\left\{\check{\lambda}_{1}, \ldots, \check{\lambda}_{n}\right\}$ das zugehörige System fundamental dominanter Gewichte.

Harder und Stuhler definieren für die Bildung des Komplementärpolyeders ([HS03, Definition 5.12]):

$$
d(C):=\sum_{i=1}^{n} \operatorname{deg}\left(\underline{\underline{u}}_{\alpha_{i}}\right) \check{\lambda}_{i} \in X_{*}(T) \oplus_{\mathbb{Z}} \mathbb{R}
$$

dabei sind die $\alpha_{i}$ die Wurzeln zu den Wänden eines Zimmers $C$, und $\underline{\underline{u}}_{\alpha_{i}}$ ist das Arakelov-Bündel der Lie-Algebra der Wurzeluntergruppe. Der Grad eines Arakelov-Bündels $L$ wurde als $\operatorname{deg}(L):=-\sum_{v \in X} \log \|a\|_{v}$ in Definition 4.15 festgelegt; dabei ist $a$ ein beliebiges Element aus $L$.

Eine ganze Struktur auf den Arakelov-Bündeln entsteht aus einer ganzen Struktur auf den Oktaven, indem in der Automorphismengruppe die (eindeutig bestimmte) ganze Struktur gewählt wird, die mit der ganzen Struktur auf den Oktaven verträglich ist, und diese ganze Struktur wird dann auf die Lie-Algebren übertragen.

In der Beschreibung durch Normen wird eine ganze Struktur als Einheitskugel zu der betreffenden Norm beschrieben.

Für eine nicht-archimedische Stelle $p$ ist die standard-ganze Struktur auf den Oktaven als die Oktav-Algebra mit $p$-ganzen Einträgen $C\left(\mathcal{O}_{p}\right)$ gegeben. Im Fall der archimedischen Stelle wird $C$ als 8-dimensionaler reeller Vektorraum mit kanonischem Skalarprodukt aufgefasst. Die zugehörige Einheitskugel stellt die standard-ganze Struktur dar.

Genau das kanonische Skalarprodukt bleibt unter der Operation der standard-maximal-kompakten Untergruppe von $G$ invariant: Dass dieses Skalarprodukt eine selbst-duale Algebra-Norm auf den Oktaven darstellt, geht aus GY03, Abschnitt 14.2] hervor. Korollar 14.5 dort bringt die Bijektion der Normen zu den maximal kompakten Untergruppen. Die maximal kompakte Untergruppe $K_{0} \subset G$ zur Standard-Norm entsteht als Schnitt von $G$ mit der $\mathrm{O}_{8}$, denn $K_{0}$ kann natürlich auch nur Elemente enthalten, deren Eigenwerte sämtlich dem Betrage nach 1 sind; die Inklusion in der anderen Richtung ist offensichtlich.

Die Standard-Involution, die auf Seite 58 definiert wurde, lässt erkennbar genau den Schnitt von $\mathfrak{g}$ mit der Lie-Algebra $\mathfrak{s o}_{8}$ invariant. Daher decken sich beide Beschreibungen; durch die Renormalisierung wurde also einzig 
dafür gesorgt, dass auch die Zahlenwerte der Normen auf der Lie-Algebra übereinstimmen.

Eine Einheitskugel o zu einem beliebigen speziellen Punkt (vgl. Seite 44) im Gebäude ist durch Multiplikation der Standard-Einheitskugel mit einem Element von $G$ bestimmt, das dem ausgewählten speziellen Punkt im Gebäude zugeordnet ist, in dem Sinne, dass seine Operation den Transfer vom Nullpunkt zum ausgewählten Punkt beschreibt.

Für das Standardapartment wird dieser Transport durch ein Element $t_{f, g}$ des Standardtorus dargestellt. Die Einheitskugel zum Punkt $(f, g)$ im Standardapartment der Normen ist daher für eine beliebige Norm $v$ aus $X$

$$
t_{f, g} o_{v} .
$$

Auf die Lie-Algebren-Ebene übertragen ist festzuhalten: Konjugation eines Elementes $x_{\eta}$ aus $\underline{U}_{\eta}$ mit $t_{f, g}$ entspricht der Multiplikation von $x_{\eta}$ mit $\eta\left(t_{f, g}\right)$. Da die Einheitskugel für eine abgeänderte Bewertung auf LieAlgebren-Ebene durch Konjugation entsteht, kann die Änderung der Bewertung durch Konjugation mit dem Inversen beschrieben werden. Die Norm von $x_{\eta}$ bezüglich der Stelle $v$ ändert sich daher um den Faktor

$$
\frac{1}{\left\|\eta\left(t_{f, g}\right)\right\|_{v}}
$$

im Vergleich zum Zahlenwert der Standardnorm.

Für die Darstellung einer Wurzel $\eta=\sum_{i} \xi_{i} \alpha_{i}$ mit Bezug auf die Wurzelbasis $\left\{\alpha_{1}, \ldots, \alpha_{n}\right\}$ gilt $\eta\left(t_{f, g}\right)=\sum_{i} \xi_{i} \alpha_{i}\left(t_{f, g}\right)$. Daher reicht es aus, die Wirkung des Toruselements auf der Wurzelbasis zu kennen.

Auf dem Standardapartment gilt nun sogar, dass bei Betrachtung eines festen $t_{f, g}$, also eines festen Punktes in jedem $p$-Apartment, der $p$-Anteil an $d(C)$ für jedes der Zimmer gleich ist. Dies ergibt sich aus dem folgenden Lemma. Dabei bezeichne $\alpha_{C, i}$ die Wurzel $\alpha_{i}$ zum Zimmer $C$, und in leicht missbräuchlicher Notation stehe $\left\|\underline{\underline{u}}_{\alpha_{C, i}}\right\|_{v}$ für die Norm eines beliebigen Elementes aus dem Arakelov-Bündel $\underline{\underline{u}}_{\alpha_{C, i}}$.

Lemma 11.1 Sei $v$ eine beliebige Stelle, $C$ ein Zimmer und $C^{\prime}:=\alpha_{C, k}(C)$ ein benachbartes Zimmer. Dann gilt:

$$
-\sum_{i=1}^{n} \log \left\|\underline{\underline{u}}_{\alpha_{C, i}}\right\|_{v} \check{\lambda}_{C, i}=-\sum_{i=1}^{n} \log \left\|\underline{\underline{u}}_{\alpha_{C^{\prime}, i}}\right\|_{v} \check{\lambda}_{C^{\prime}, i} .
$$

Beweis. Sei ohne Einschränkung $k=1$. Für die fundamentalen Wurzeln zu den beiden Zimmern gilt: $\alpha_{C^{\prime}, i}=s_{\alpha_{C, 1}}\left(\alpha_{C, i}\right)$, ebenso gilt $\check{\lambda}_{C^{\prime}, i}=s_{\alpha_{C, 1}}\left(\check{\lambda}_{C, i}\right)$. Damit ergibt sich: 


$$
\begin{aligned}
& -\sum_{i=1}^{n} \log \left\|\underline{\underline{u}}_{\alpha_{C^{\prime}, i}}\right\|_{v} \check{\lambda}_{C^{\prime}, i} \\
& =-\sum_{i=1}^{n} \log \left\|\underline{\underline{u}}_{s_{\alpha_{C, 1}}\left(\alpha_{C, i}\right)}\right\|_{v} s_{\alpha_{C, 1}}\left(\check{\lambda}_{C, i}\right) \\
& =-\log \left\|\underline{\underline{u}}_{-\alpha_{C, 1}}\right\|_{v}\left(\check{\lambda}_{C, 1}-\left\langle\alpha_{C, 1}, \check{\lambda}_{C, 1}\right\rangle \alpha_{C, 1}^{\vee}\right) \\
& -\sum_{i=2}^{n}\left(\log \left\|\underline{\underline{u}}_{\alpha_{C, i}}\right\|_{v}-\left\langle\alpha_{C, i}, \alpha_{C, 1}^{\vee}\right\rangle \log \left\|\underline{\underline{u}}_{\alpha_{C, 1}}\right\|_{v}\right)(\check{\lambda}_{C, i}-\underbrace{\left\langle\alpha_{C, 1}, \check{\lambda}_{C, i}\right\rangle}_{=0} \alpha_{C, 1}^{\vee}) \\
& =\log \left\|\underline{\underline{u}}_{\alpha_{C, 1}}\right\|{ }_{v}\left(\check{\lambda}_{C, 1}-\alpha_{C, 1}^{\vee}\right) \\
& -\sum_{i=2}^{n}\left(\log \left\|\underline{\underline{u}}_{\alpha_{C, i}}\right\|_{v}-\left\langle\alpha_{C, i}, \alpha_{C, 1}^{\vee}\right\rangle \log \left\|\underline{\underline{u}}_{\alpha_{C, 1}}\right\|_{v}\right) \check{\lambda}_{C, i} \\
& =-\sum_{i=1}^{n} \log \left\|\underline{\underline{u}}_{\alpha_{C, i}}\right\|_{v} \check{\lambda}_{C, i}+2 \log \left\|\underline{\underline{u}}_{\alpha_{C, 1}}\right\| \check{\lambda}_{v}-\log \left\|\underline{\underline{u}}_{\alpha_{C, 1}}\right\|{ }_{v} \alpha_{C, 1}^{\vee} \\
& +\sum_{i=2}^{n}\left\langle\alpha_{C, i}, \alpha_{C, 1}^{\vee}\right\rangle \log \left\|\underline{\underline{u}}_{\alpha_{C, 1}}\right\|_{v} \check{\lambda}_{C, i}
\end{aligned}
$$

Wegen $\left\langle\alpha_{C, i}, \check{\lambda}_{C, j}\right\rangle=\delta_{i j}$ und $\left\langle\alpha_{C, 1}, \alpha_{C, 1}^{\vee}\right\rangle=2$ gilt

$$
\begin{array}{r}
\left\langle\alpha_{C, j}, 2 \log \left\|\underline{\underline{u}}_{\alpha_{C, 1}}\right\| \check{\check{\lambda}}_{C, 1}-\log \left\|\underline{\underline{u}}_{\alpha_{C, 1}}\right\|_{v} \alpha_{C, 1}^{\vee}+\right. \\
\left.\sum_{i=2}^{n}\left\langle\alpha_{C, i}, \alpha_{C, 1}^{\vee}\right\rangle \log \left\|\underline{\underline{u}}_{\alpha_{C, 1}}\right\|_{v} \check{\lambda}_{C, i}\right\rangle=0 .
\end{array}
$$

für jedes $j$. Damit ist

$$
-\sum_{i=1}^{n} \log \left\|\underline{\underline{u}}_{\alpha_{C^{\prime}, i}}\right\| \check{\check{\lambda}}_{C^{\prime}, i}=-\sum_{i=1}^{n} \log \left\|\underline{\underline{u}}_{\alpha_{C, i}}\right\|{ }_{v} \check{\lambda}_{C, i}
$$

wie behauptet.

Daraus folgt außerdem:

Korollar 11.2 Sei $\left(C ;\|\cdot\|_{v}: v \in X\right)$ eine ganze Struktur auf $\mathfrak{g}$, bei der sämtliche Normen aus dem jeweiligen Standardapartment stammen. Dann entartet das Komplementärpolyeder zu einem Punkt.

Satz 11.3 Sei $\left(C ;\|\cdot\|_{v}: v \in X\right)$ eine ganze Struktur auf $\mathfrak{g}$, bei der sämtliche Normen aus dem jeweiligen Standardapartment stammen. Wenn es ein Toruselement gibt, das jede der verwendeten Normen beschreibt, das heißt also, wenn das Arakelov-Bündel vollständig kohärente Normen hat, dann liegt das (zum Punkt entartete) Komplementärpolyeder auf dem Nullpunkt. 
Beweis. Der Satz ergibt sich sofort aus dem zuvor Hergeleiteten sowie der Produktformel (4.1).

Nun stellt sich die Frage, für welche Verschiebung des Komplementärpolyeder-Punktes das Abändern einer einzigen Norm zu einer Stelle $v$ sorgt. Nach Korollar 11.2 kann man ohne Einschränkung die Verschiebung von $d(C)$ für das Zimmer zu den Wurzeln $\alpha$ und $\beta$ betrachten.

Betrachtet man die Situation im nicht-archimedischen Fall von der Bewertungsseite her, ist das Verschieben additiv. Daher genügt es, die Wirkung von $\hat{t}_{r, s}$ für $(r, s)=(1,0)$ und $(r, s)=(0,1)$ zu kennen. Man berechnet unter Beachtung von (11.1):

$$
\begin{aligned}
& -\log \left\|\hat{t}_{1,0}^{-1} \underline{\underline{u}}_{\alpha} \hat{t}_{1,0}\right\|_{v} \check{\lambda}_{\alpha}-\log \left\|\hat{t}_{1,0}^{-1} \underline{\underline{u}}_{\beta} \hat{t}_{1,0}\right\|_{v} \check{\lambda}_{\beta} \\
= & v\left(\alpha\left(\hat{t}_{1,0}\right)\right) \check{\lambda}_{\alpha}+v\left(\beta\left(\hat{t}_{1,0}\right)\right) \check{\lambda}_{\beta} \\
= & 2 \check{\lambda}_{\alpha}-\check{\lambda}_{\beta} \\
= & \check{\alpha}=a^{\vee}
\end{aligned}
$$

und

$$
\begin{aligned}
& -\log \left\|\hat{t}_{0,1}^{-1} \underline{\underline{u}}_{\alpha} \hat{t}_{0,1}\right\|_{v} \check{\lambda}_{\alpha}+\log \left\|\hat{t}_{0,1}^{-1} \underline{\underline{u}}_{\beta} \hat{t}_{0,1}\right\|_{v} \check{\lambda}_{\beta} \\
= & v\left(\alpha\left(\hat{t}_{0,1}\right)\right) \check{\lambda}_{\alpha}+v\left(\beta\left(\hat{t}_{0,1}\right)\right) \check{\lambda}_{\beta} \\
= & \check{\lambda}_{\alpha}=b^{\vee} .
\end{aligned}
$$

(Man vergleiche mit den Abbildungen auf Seite 42,) Die Rechnungen verlaufen für die archimedische Bewertung ganz entsprechend. Insbesondere ist also die Bahn der Verschiebung für jeden einzelnen Parameter eine Gerade, unabhängig von der Primstelle.

Wegen dieses gut kontrollierbaren Verhaltens der Torusoperation ist es möglich, die Verschiebung auch in Termen der Grade von $V$ und $W$ aus der invarianten Flagge zur Borelgruppe zu beschreiben (siehe Seite 67 und Satz 10.1). Beim Standardzimmer bewirkt die Multiplikation von $V=\left\langle e_{11} \boldsymbol{a}\right\rangle$ mit $\hat{t}_{r, s}^{-1}$ eine Änderung der Bewertung des Grades um $-s$, bei $W / V=$ $\left\langle e_{11} \boldsymbol{a}, e_{12} \boldsymbol{a}\right\rangle /\left\langle e_{11} \boldsymbol{a}\right\rangle$ eine Änderung um $-r-s$. Daher kann man das Verschieben des Komplementärpolyeders auch durch eine geeignete Linearkombination der Veränderungen der Grade von $V$ und $W$ darstellen. Und weil sich die Wirkungen der Torusoperation auf den Flaggenräumen beim Wechsel zu einem anderen Zimmer in gleicher Weise verändern wie die Wirkungen auf den Wurzeln, die die Zimmer definieren, gilt diese Aussage für alle Zimmer. Für das Standardzimmer $C$ berechnet man:

$$
\begin{aligned}
d\left(\hat{t}_{r, s}^{-1} C\right) & =\left(\operatorname{deg}\left(\hat{t}_{r, s}^{-1} V\right)-\operatorname{deg}\left(\hat{t}_{r, s}^{-1}(W / V)\right)\right) a^{\vee}-\operatorname{deg}\left(\hat{t}_{r, s}^{-1} V\right) b^{\vee} \\
& =\left(\operatorname{deg}\left(\hat{t}_{r, s}^{-1} V\right)-\operatorname{deg}\left(\hat{t}_{r, s}^{-1}(W / V)\right)-2 \operatorname{deg}\left(\hat{t}_{r, s}^{-1} V\right)\right) \check{\alpha}-\operatorname{deg}\left(\hat{t}_{r, s}^{-1} V\right) \check{\beta} \\
& =\left(-\operatorname{deg}\left(\hat{t}_{r, s}^{-1}(W / V)\right)-\operatorname{deg}\left(\hat{t}_{r, s}^{-1} V\right)\right) \check{\alpha}-\operatorname{deg}\left(\hat{t}_{r, s}^{-1} V\right) \check{\beta} .
\end{aligned}
$$


Damit folgt allgemein der

Satz 11.4 Sei $Z$ ein Zimmer im endlichen Apartment von Typ $\mathrm{G}_{2} z u G$, und sei $B$ die Borelgruppe $z u Z$. Sei $\gamma$ die lange und $\delta$ die kurze Wurzel in der Wurzelbasis zu Z. Seit ein Element des Standardtorus. Sei schließlich $0 \subset V \subset W$ der charakterisierende Teil der Flagge zu B gemäß Satz 10.1. Dann gilt:

$$
d(t Z)=(-\operatorname{deg}(t(W / V))-\operatorname{deg}(t V)) \check{\alpha}-\operatorname{deg}(t V) \check{\beta} .
$$

In gewisser Weise mag das Resultat, dass die Komplementärpolyeder entarten, nicht besonders spannend erscheinen, es ist jedoch in einem größeren Zusammenhang insofern gut zu erklären, als die zu den Normen gehörenden Gitter, solange man sich mit den Normen nur im Standardapartment bewegt, orthogonale Basen besitzen. Daraus ergibt sich, dass die Normen auf den Wurzeluntergruppen linear in Bezug auf den Raum der Wurzeln sind. Deswegen können sich keine besonderen Formen als Komplementärpolyeder bilden.

Es besteht die Hoffnung, dass die viele Einzelheiten beleuchtenden Untersuchungen in dieser Arbeit bei weiteren Forschungen zum allgemeinen Verständnis der Gestalt der Komplementärpolyeder im gesamten Gebäude beitragen können. 


\section{A Anhang}

Auch im Anhang sind in den großen Matrizen die Nullen durch Punkte ersetzt.

\section{A.1 Berechnung der weiteren Schnittbedingungen aus Ka- pitel 8}

- Zur Wurzel $-\alpha-3 \beta$ bzw. zum Charakter $[r-s]$ : Konjugation des Standardtorus mit

$$
\left(\begin{array}{cccccccc}
1 & \cdot & \cdot & \cdot & \cdot & \cdot & \cdot & \cdot \\
\cdot & 1 & \cdot & \cdot & \cdot & \cdot & \cdot & \cdot \\
\cdot & \cdot & 1 & \cdot & -b & \cdot & \cdot & \cdot \\
\cdot & \cdot & \cdot & 1 & \cdot & \cdot & \cdot & \cdot \\
\cdot & \cdot & \cdot & \cdot & 1 & \cdot & \cdot & \cdot \\
\cdot & \cdot & \cdot & b & \cdot & 1 & \cdot & \cdot \\
\cdot & \cdot & \cdot & \cdot & \cdot & \cdot & 1 & \cdot \\
\cdot & \cdot & \cdot & \cdot & \cdot & \cdot & \cdot & 1
\end{array}\right)
$$

liefert den Torus

$$
\left\{\left(\begin{array}{cccccccc}
1 & \cdot & \cdot & \cdot & \cdot & \cdot & \cdot & \cdot \\
\cdot & 1 & \cdot & \cdot & \cdot & \cdot & \cdot & \cdot \\
\cdot & \cdot & f & \cdot & -b(g-f) & \cdot & \cdot & \cdot \\
\cdot & \cdot & \cdot & \frac{1}{f} & \cdot & \cdot & \cdot & \cdot \\
\cdot & \cdot & \cdot & \cdot & g & \cdot & \cdot & \cdot \\
\cdot & \cdot & \cdot & b\left(\frac{1}{f}-\frac{1}{g}\right) & \cdot & \frac{1}{g} & \cdot & \cdot \\
\cdot & \cdot & \cdot & \cdot & \cdot & \cdot & f g & \cdot \\
\cdot & \cdot & \cdot & \cdot & \cdot & \cdot & \cdot & \frac{1}{f g}
\end{array}\right) \mid f, g \in K^{*}\right\} .
$$

Dieser hat zur Basis

$$
\left\{e_{11}, e_{22}, e_{12}, e_{21}+b \cdot e_{22} \boldsymbol{a}, e_{11} \boldsymbol{a}-b \cdot e_{12}, e_{22} \boldsymbol{a}, e_{12} \boldsymbol{a}, e_{21} \boldsymbol{a}\right\}
$$

Diagonalgestalt.

Der Schnittbereich des zugehörigen Apartments ergibt sich aus der Bedingung $r-s \leq v(b)$.

- Zur Wurzel $-2 \alpha-3 \beta$ bzw. zum Charakter $[-r-2 s]$ : Konjugation des Standardtorus mit

$$
\left(\begin{array}{rrrrrrrr}
1 & \cdot & \cdot & \cdot & \cdot & \cdot & \cdot & \cdot \\
\cdot & 1 & \cdot & \cdot & \cdot & \cdot & \cdot & \cdot \\
\cdot & \cdot & 1 & \cdot & \cdot & \cdot & \cdot & \cdot \\
\cdot & \cdot & \cdot & 1 & \cdot & \cdot & \cdot & \cdot \\
\cdot & \cdot & \cdot & \cdot & 1 & \cdot & \cdot & \cdot \\
\cdot & \cdot & \cdot & \cdot & \cdot & 1 & -b & \cdot \\
\cdot & \cdot & \cdot & \cdot & \cdot & \cdot & 1 & \cdot \\
\cdot & \cdot & \cdot & \cdot & -b & \cdot & \cdot & 1
\end{array}\right)
$$


liefert den Torus

$$
\left\{\left(\begin{array}{cccccccc}
1 & \cdot & \cdot & \cdot & \cdot & \cdot & \cdot & \cdot \\
\cdot & 1 & \cdot & \cdot & \cdot & \cdot & \cdot & \cdot \\
\cdot & \cdot & f & \cdot & \cdot & \cdot & \cdot & \cdot \\
\cdot & \cdot & \cdot & \frac{1}{f} & \cdot & \cdot & \cdot & \cdot \\
\cdot & \cdot & \cdot & \cdot & g & \cdot & \cdot & \cdot \\
\cdot & \cdot & \cdot & \cdot & \cdot & \frac{1}{g} & -b\left(f g-\frac{1}{g}\right) & \cdot \\
\cdot & \cdot & \cdot & \cdot & \cdot & \cdot & f g & \cdot \\
\cdot & \cdot & \cdot & \cdot & -b\left(g-\frac{1}{f g}\right) & \cdot & \cdot & \frac{1}{f g}
\end{array}\right) \mid f, g \in K^{*}\right\}
$$

Dieser hat zur Basis

$$
\left\{e_{11}, e_{22}, e_{12}, e_{21}, e_{11} \boldsymbol{a}-b \cdot e_{21} \boldsymbol{a}, e_{22} \boldsymbol{a}, e_{12} \boldsymbol{a}-b \cdot e_{22} \boldsymbol{a}, e_{21} \boldsymbol{a}\right\}
$$

Diagonalgestalt.

Der Schnittbereich des zugehörigen Apartments ergibt sich aus der Bedingung $-r-2 s \leq v(b) \Leftrightarrow r+2 s \geq-v(b)$.

- Zur Wurzel $-\alpha$ bzw. zum Charakter $[-2 r-s]$ : Konjugation des Standardtorus mit

$$
\left(\begin{array}{cccccccc}
1 & \cdot & \cdot & \cdot & \cdot & \cdot & \cdot & \cdot \\
\cdot & 1 & \cdot & \cdot & \cdot & \cdot & \cdot & \cdot \\
\cdot & \cdot & 1 & \cdot & \cdot & \cdot & \cdot & \cdot \\
\cdot & \cdot & \cdot & 1 & \cdot & \cdot & -b & \cdot \\
\cdot & \cdot & \cdot & \cdot & 1 & \cdot & \cdot & \cdot \\
\cdot & \cdot & \cdot & \cdot & \cdot & 1 & \cdot & \cdot \\
\cdot & \cdot & \cdot & \cdot & \cdot & \cdot & 1 & \cdot \\
\cdot & \cdot & -b & \cdot & \cdot & \cdot & \cdot & 1
\end{array}\right)
$$

liefert den Torus

$$
\left\{\left(\begin{array}{cccccccc}
1 & \cdot & \cdot & \cdot & \cdot & \cdot & \cdot & \cdot \\
\cdot & 1 & \cdot & \cdot & \cdot & \cdot & \cdot & \cdot \\
\cdot & \cdot & f & \cdot & \cdot & \cdot & \cdot & \cdot \\
\cdot & \cdot & \cdot & \frac{1}{f} & \cdot & \cdot & -b\left(f g-\frac{1}{f}\right) & \cdot \\
\cdot & \cdot & \cdot & \cdot & g & \cdot & \cdot & \cdot \\
\cdot & \cdot & \cdot & \cdot & \cdot & \frac{1}{g} & \cdot & \cdot \\
\cdot & \cdot & \cdot & \cdot & \cdot & \cdot & f g & \cdot \\
\cdot & \cdot & -b\left(f-\frac{1}{f g}\right) & \cdot & \cdot & \cdot & \cdot & \frac{1}{f g}
\end{array}\right) \mid f, g \in K^{*}\right\} .
$$

Dieser hat zur Basis

$$
\left\{e_{11}, e_{22}, e_{12}-b \cdot e_{21} \boldsymbol{a}, e_{21}, e_{11} \boldsymbol{a}, e_{22} \boldsymbol{a}, e_{12} \boldsymbol{a}-b \cdot e_{21}, e_{21} \boldsymbol{a}\right\}
$$

Diagonalgestalt.

Der Schnittbereich des zugehörigen Apartments ergibt sich aus der Bedingung $-2 r-s \leq v(b) \Leftrightarrow 2 r+s \geq-v(b)$. 
- Zur Wurzel $\alpha+3 \beta$ bzw. zum Charakter $[-r+s]$ : Konjugation des Standardtorus mit

$$
\left(\begin{array}{cccccccc}
1 & \cdot & \cdot & \cdot & \cdot & \cdot & \cdot & \cdot \\
\cdot & 1 & \cdot & \cdot & \cdot & \cdot & \cdot & \cdot \\
\cdot & \cdot & 1 & \cdot & \cdot & \cdot & \cdot & \cdot \\
\cdot & \cdot & \cdot & 1 & \cdot & -b & \cdot & \cdot \\
\cdot & \cdot & b & \cdot & 1 & \cdot & \cdot & \cdot \\
\cdot & \cdot & \cdot & \cdot & \cdot & 1 & \cdot & \cdot \\
\cdot & \cdot & \cdot & \cdot & \cdot & \cdot & 1 & \cdot \\
\cdot & \cdot & \cdot & \cdot & \cdot & \cdot & \cdot & 1
\end{array}\right)
$$

liefert den Torus

$$
\left\{\left(\begin{array}{cccccccc}
1 & \cdot & \cdot & \cdot & \cdot & \cdot & \cdot & \cdot \\
\cdot & 1 & \cdot & \cdot & \cdot & \cdot & \cdot & \cdot \\
\cdot & \cdot & f & \cdot & \cdot & \cdot & \cdot & \cdot \\
\cdot & \cdot & \cdot & \frac{1}{f} & \cdot & -b\left(\frac{1}{g}-\frac{1}{f}\right) & \cdot & \cdot \\
\cdot & \cdot & b(f-g) & \cdot & g & \cdot & \cdot & \cdot \\
\cdot & \cdot & \cdot & \cdot & \cdot & \frac{1}{g} & \cdot & \cdot \\
\cdot & \cdot & \cdot & \cdot & \cdot & \cdot & f g & \cdot \\
\cdot & \cdot & \cdot & \cdot & \cdot & \cdot & \cdot & \frac{1}{f g}
\end{array}\right) \mid f, g \in K^{*}\right\} .
$$

Dieser hat zur Basis

$$
\left\{e_{11}, e_{22}, e_{12}+b \cdot e_{11} \boldsymbol{a}, e_{21}, e_{11} \boldsymbol{a}, e_{22} \boldsymbol{a}-b \cdot e_{21}, e_{12} \boldsymbol{a}, e_{21} \boldsymbol{a}\right\}
$$

Diagonalgestalt.

Der Schnittbereich des zugehörigen Apartments ergibt sich aus der Bedingung $-r+s \leq v(b) \Leftrightarrow r-s \geq-v(b)$.

- Zur Wurzel $2 \alpha+3 \beta$ bzw. zum Charakter $[r+2 s]$ : Konjugation des Standardtorus mit

$$
\left(\begin{array}{llllllll}
1 & \cdot & \cdot & \cdot & \cdot & \cdot & \cdot & \cdot \\
\cdot & 1 & \cdot & \cdot & \cdot & \cdot & \cdot & \cdot \\
\cdot & \cdot & 1 & \cdot & \cdot & \cdot & \cdot & \cdot \\
\cdot & \cdot & \cdot & 1 & \cdot & \cdot & \cdot & \cdot \\
\cdot & \cdot & \cdot & \cdot & 1 & \cdot & \cdot & b \\
\cdot & \cdot & \cdot & \cdot & \cdot & 1 & \cdot & \cdot \\
\cdot & \cdot & \cdot & \cdot & \cdot & b & 1 & \cdot \\
\cdot & \cdot & \cdot & \cdot & \cdot & \cdot & \cdot & 1
\end{array}\right)
$$


liefert den Torus

$$
\left\{\left(\begin{array}{cccccccc}
1 & \cdot & \cdot & \cdot & \cdot & \cdot & \cdot & \cdot \\
\cdot & 1 & \cdot & \cdot & \cdot & \cdot & \cdot & \cdot \\
\cdot & \cdot & f & \cdot & \cdot & \cdot & \cdot & \cdot \\
\cdot & \cdot & \cdot & \frac{1}{f} & \cdot & \cdot & \cdot & \cdot \\
\cdot & \cdot & \cdot & \cdot & g & \cdot & \cdot & b\left(\frac{1}{f g}-g\right) \\
\cdot & \cdot & \cdot & \cdot & \cdot & \frac{1}{g} & \cdot & \cdot \\
\cdot & \cdot & \cdot & \cdot & \cdot & b\left(\frac{1}{g}-f g\right) & f g & \cdot \\
\cdot & \cdot & \cdot & \cdot & \cdot & \cdot & \cdot & \frac{1}{f g}
\end{array}\right) \mid f, g \in K^{*}\right\} .
$$

Dieser hat zur Basis

$$
\left\{e_{11}, e_{22}, e_{12}, e_{21}, e_{11} \boldsymbol{a}, e_{22} \boldsymbol{a}+b \cdot e_{12} \boldsymbol{a}, e_{12} \boldsymbol{a}, e_{21} \boldsymbol{a}+b \cdot e_{11} \boldsymbol{a}\right\}
$$

Diagonalgestalt.

Der Schnittbereich des zugehörigen Apartments ergibt sich aus der Bedingung $r+2 s \leq v(b)$.

Auch bei den Wurzeluntergruppen zu den anderen sechs Wurzeln zählen dieselben Argumente (insbesondere ist modulo $\mathcal{O}$ auch hier der Basiswechsel an der verschobenen Stelle eindeutig festgelegt, falls überhaupt möglich, da die Determinante der Basiswechselmatrix wiederum nur durch die Diagonaleinträge bestimmt wird).

- Zur Wurzel $-\alpha-2 \beta$ bzw. zum Charakter $[-s]$ : Konjugation des Standardtorus mit

$$
\left(\begin{array}{cccccccc}
1 & \cdot & \cdot & \cdot & -b & \cdot & \cdot & \cdot \\
\cdot & 1 & \cdot & \cdot & b & \cdot & \cdot & \cdot \\
\cdot & \cdot & 1 & \cdot & \cdot & \cdot & b & \cdot \\
\cdot & \cdot & \cdot & 1 & \cdot & \cdot & \cdot & \cdot \\
\cdot & \cdot & \cdot & \cdot & 1 & \cdot & \cdot & \cdot \\
b & -b & \cdot & \cdot & -b^{2} & 1 & \cdot & \cdot \\
\cdot & \cdot & \cdot & \cdot & \cdot & \cdot & 1 & \cdot \\
\cdot & \cdot & \cdot & b & \cdot & \cdot & \cdot & 1
\end{array}\right)
$$

liefert den Torus aller Elemente

$$
\left(\begin{array}{cccccccc}
1 & \cdot & \cdot & \cdot & -b(g-1) & \cdot & \cdot & \cdot \\
\cdot & 1 & \cdot & \cdot & b(g-1) & \cdot & \cdot & \cdot \\
\cdot & \cdot & f & \cdot & \cdot & \cdot & b(f g-f) & \cdot \\
\cdot & \cdot & \cdot & \frac{1}{f} & \cdot & \cdot & \cdot & \cdot \\
\cdot & \cdot & \cdot & \cdot & g & \cdot & \cdot & \cdot \\
b\left(1-\frac{1}{g}\right) & -b\left(1-\frac{1}{g}\right) & \cdot & \cdot & -b^{2}\left(g-2+\frac{1}{g}\right) & \frac{1}{g} & \cdot & \cdot \\
\cdot & \cdot & \cdot & \cdot & \cdot & \cdot & \cdot \\
\cdot & \cdot & \cdot & b\left(\frac{1}{f}-\frac{1}{f g}\right) & \cdot & \cdot & \cdot & \frac{1}{f g}
\end{array}\right)
$$

für $f, g \in K^{*}$. Dieser hat zur Basis 


$$
\begin{aligned}
& \left\{e_{11}+b \cdot e_{22} \boldsymbol{a}, e_{22}-b \cdot e_{22} \boldsymbol{a}, e_{12}, e_{21}+b \cdot e_{21} \boldsymbol{a},\right. \\
& \left.\quad e_{11} \boldsymbol{a}-b \cdot e_{11}+b \cdot e_{22}-b^{2} \cdot e_{22} \boldsymbol{a}, e_{22} \boldsymbol{a}, e_{12} \boldsymbol{a}+b \cdot e_{12}, e_{21} \boldsymbol{a}\right\}
\end{aligned}
$$

Diagonalgestalt.

Der Schnittbereich des zugehörigen Apartments ergibt sich aus der Bedingung $-s \leq v(b) \Leftrightarrow s \geq-v(b)$.

- Zur Wurzel $-\alpha-\beta$ bzw. zum Charakter $[-r-s]$ : Konjugation des Standardtorus mit

$$
\left(\begin{array}{rrrrrrrr}
1 & \cdot & \cdot & \cdot & \cdot & \cdot & b & \cdot \\
\cdot & 1 & \cdot & \cdot & \cdot & \cdot & -b & \cdot \\
\cdot & \cdot & 1 & \cdot & \cdot & \cdot & \cdot & \cdot \\
\cdot & \cdot & \cdot & 1 & b & \cdot & \cdot & \cdot \\
\cdot & \cdot & \cdot & \cdot & 1 & \cdot & \cdot & \cdot \\
\cdot & \cdot & -b & \cdot & \cdot & 1 & \cdot & \cdot \\
\cdot & \cdot & \cdot & \cdot & \cdot & \cdot & 1 & \cdot \\
b & -b & \cdot & \cdot & \cdot & \cdot & b^{2} & 1
\end{array}\right)
$$

liefert den Torus aller Elemente

$$
\left(\begin{array}{cccccccc}
1 & \cdot & \cdot & \cdot & \cdot & \cdot & b(f g-1) & \cdot \\
\cdot & 1 & \cdot & \cdot & \cdot & \cdot & -b(f g-1) & \cdot \\
\cdot & \cdot & f & \cdot & \cdot & \cdot & \cdot & \cdot \\
\cdot & \cdot & \cdot & \frac{1}{f} & b\left(g-\frac{1}{f}\right) & \cdot & \cdot & \cdot \\
\cdot & \cdot & \cdot & \cdot & g & \cdot & \cdot & \cdot \\
\cdot & \cdot & -b\left(f-\frac{1}{g}\right) & \cdot & \cdot & \frac{1}{g} & \cdot & \cdot \\
\cdot & \cdot & \cdot & \cdot & \cdot & \cdot & f g & \cdot \\
b\left(1-\frac{1}{f g}\right) & -b\left(1-\frac{1}{f g}\right) & \cdot & \cdot & \cdot & \cdot & b^{2}\left(f g-2+\frac{1}{f g}\right) & \frac{1}{f g}
\end{array}\right)
$$

für $f, g \in K^{*}$. Dieser hat zur Basis

$$
\begin{aligned}
& \left\{e_{11}+b \cdot e_{21} \boldsymbol{a}, e_{22}-b \cdot e_{21} \boldsymbol{a}, e_{12}-b \cdot e_{22} \boldsymbol{a}, e_{21},\right. \\
& \left.e_{11} \boldsymbol{a}+b \cdot e_{21}, e_{22} \boldsymbol{a}, e_{12} \boldsymbol{a}+b \cdot e_{11}-b \cdot e_{22}+b^{2} \cdot e_{21} \boldsymbol{a}, e_{21} \boldsymbol{a}\right\}
\end{aligned}
$$

Diagonalgestalt.

Der Schnittbereich des zugehörigen Apartments ergibt sich aus der Bedingung $-r-s \leq v(b) \Leftrightarrow r+s \geq-v(b)$.

- Zur Wurzel $\beta$ bzw. zum Charakter $[-r]$ : Konjugation des Standardtorus mit

$$
\left(\begin{array}{cccccccc}
1 & \cdot & b & \cdot & \cdot & \cdot & \cdot & \cdot \\
\cdot & 1 & -b & \cdot & \cdot & \cdot & \cdot & \cdot \\
\cdot & \cdot & 1 & \cdot & \cdot & \cdot & \cdot & \cdot \\
-b & b & -b^{2} & 1 & \cdot & \cdot & \cdot & \cdot \\
\cdot & \cdot & \cdot & \cdot & 1 & \cdot & b & \cdot \\
\cdot & \cdot & \cdot & \cdot & \cdot & 1 & \cdot & \cdot \\
\cdot & \cdot & \cdot & \cdot & \cdot & \cdot & 1 & \cdot \\
\cdot & \cdot & \cdot & \cdot & \cdot & b & \cdot & 1
\end{array}\right)
$$


liefert den Torus aller Elemente

$$
\left(\begin{array}{cccccccc}
1 & \cdot & b(f-1) & \cdot & \cdot & \cdot & \cdot & \cdot \\
\cdot & 1 & -b(f-1) & \cdot & \cdot & \cdot & \cdot & \cdot \\
\cdot & \cdot & f & \cdot & \cdot & \cdot & \cdot & \cdot \\
-b\left(1-\frac{1}{f}\right) & b\left(1-\frac{1}{f}\right) & -b^{2}\left(f-2+\frac{1}{f}\right) & \frac{1}{f} & \cdot & \cdot & \cdot & \cdot \\
\cdot & \cdot & \cdot & \cdot & g & \cdot & b(f g-g) & \cdot \\
\cdot & \cdot & \cdot & \cdot & \cdot & \frac{1}{g} & \cdot & \cdot \\
\cdot & \cdot & \cdot & \cdot & \cdot & \cdot & f g & \cdot \\
\cdot & \cdot & \cdot & \cdot & \cdot & b\left(\frac{1}{g}-\frac{1}{f g}\right) & \cdot & \frac{1}{f g}
\end{array}\right)
$$

für $f, g \in K^{*}$. Dieser hat zur Basis

$$
\begin{aligned}
\left\{e_{11}-b \cdot e_{21}, e_{22}+b \cdot e_{21},\right. & e_{12}+b \cdot e_{11}-b \cdot e_{22}-b^{2} \cdot e_{21}, e_{21}, \\
& \left.e_{11} \boldsymbol{a}, e_{22} \boldsymbol{a}+b \cdot e_{21} \boldsymbol{a}, e_{12} \boldsymbol{a}+b \cdot e_{11} \boldsymbol{a}, e_{21} \boldsymbol{a}\right\}
\end{aligned}
$$

Diagonalgestalt.

Der Schnittbereich des zugehörigen Apartments ergibt sich aus der Bedingung $-r \leq v(b) \quad \Leftrightarrow \quad r \geq-v(b)$.

- Zur Wurzel $\alpha+2 \beta$ bzw. zum Charakter [s]: Konjugation des Standardtorus mit

$$
\left(\begin{array}{rrrrrrrr}
1 & \cdot & \cdot & \cdot & \cdot & -b & \cdot & \cdot \\
\cdot & 1 & \cdot & \cdot & \cdot & b & \cdot & \cdot \\
\cdot & \cdot & 1 & \cdot & \cdot & \cdot & \cdot & \cdot \\
\cdot & \cdot & \cdot & 1 & \cdot & \cdot & \cdot & -b \\
b & -b & \cdot & \cdot & 1 & -b^{2} & \cdot & \cdot \\
\cdot & \cdot & \cdot & \cdot & \cdot & 1 & \cdot & \cdot \\
\cdot & \cdot & -b & \cdot & \cdot & \cdot & 1 & \cdot \\
\cdot & \cdot & \cdot & \cdot & \cdot & \cdot & \cdot & 1
\end{array}\right)
$$

liefert den Torus aller Elemente

$$
\left(\begin{array}{cccccccc}
1 & \cdot & \cdot & \cdot & \cdot & -b\left(\frac{1}{g}-1\right) & \cdot & \cdot \\
\cdot & 1 & \cdot & \cdot & \cdot & b\left(\frac{1}{g}-1\right) & \cdot & \cdot \\
\cdot & \cdot & f & \cdot & \cdot & \cdot & \cdot & \cdot \\
\cdot & \cdot & \cdot & \frac{1}{f} & \cdot & \cdot & \cdot & -b\left(\frac{1}{f g}-\frac{1}{f}\right) \\
b(1-g) & -b(1-g) & \cdot & \cdot & g & -b^{2}\left(\frac{1}{g}-2+g\right) & \cdot & \cdot \\
\cdot & \cdot & \cdot & \cdot & \cdot & \frac{1}{g} & \cdot & \cdot \\
\cdot & \cdot & -b(f-f g) & \cdot & \cdot & \cdot & f g & \cdot \\
\cdot & \cdot & \cdot & \cdot & \cdot & \cdot & \cdot & \frac{1}{f g}
\end{array}\right)
$$

für $f, g \in K^{*}$. Dieser hat zur Basis

$$
\begin{aligned}
& \left\{e_{11}+b \cdot e_{11} \boldsymbol{a}, e_{22}-b \cdot e_{11} \boldsymbol{a}, e_{12}-b \cdot e_{12} \boldsymbol{a}, e_{21},\right. \\
& \left.\quad e_{11} \boldsymbol{a}, e_{22} \boldsymbol{a}-b \cdot e_{11}+b \cdot e_{22}-b^{2} \cdot e_{11} \boldsymbol{a}, e_{12} \boldsymbol{a}, e_{21} \boldsymbol{a}-b \cdot e_{21}\right\}
\end{aligned}
$$

Diagonalgestalt. 
Der Schnittbereich des zugehörigen Apartments ergibt sich aus der Bedingung $s \leq v(b)$.

- Zur Wurzel $\alpha+\beta$ bzw. zum Charakter $[r+s]$ : Konjugation des Standardtorus mit

$$
\left(\begin{array}{rrrrrrrr}
1 & \cdot & \cdot & \cdot & \cdot & \cdot & \cdot & -b \\
\cdot & 1 & \cdot & \cdot & \cdot & \cdot & \cdot & b \\
\cdot & \cdot & 1 & \cdot & \cdot & b & \cdot & \cdot \\
\cdot & \cdot & \cdot & 1 & \cdot & \cdot & \cdot & \cdot \\
\cdot & \cdot & \cdot & -b & 1 & \cdot & \cdot & \cdot \\
\cdot & \cdot & \cdot & \cdot & \cdot & 1 & \cdot & \cdot \\
-b & b & \cdot & \cdot & \cdot & \cdot & 1 & b^{2} \\
\cdot & \cdot & \cdot & \cdot & \cdot & \cdot & \cdot & 1
\end{array}\right)
$$

liefert den Torus aller Elemente

$$
\left(\begin{array}{cccccccc}
1 & \cdot & \cdot & \cdot & \cdot & \cdot & \cdot & -b\left(\frac{1}{f g}-1\right) \\
\cdot & 1 & \cdot & \cdot & \cdot & \cdot & \cdot & b\left(\frac{1}{f g}-1\right) \\
\cdot & \cdot & f & \cdot & \cdot & b\left(\frac{1}{g}-f\right) & \cdot & \cdot \\
\cdot & \cdot & \cdot & \frac{1}{f} & \cdot & \cdot & \cdot & \cdot \\
\cdot & \cdot & \cdot & -b\left(\frac{1}{f}-g\right) & g & \cdot & \cdot & \cdot \\
\cdot & \cdot & \cdot & \cdot & \cdot & \frac{1}{g} & \cdot & \cdot \\
-b(1-f g) & b(1-f g) & \cdot & \cdot & \cdot & \cdot & f g & b^{2}\left(\frac{1}{f g}-2+f g\right) \\
\cdot & \cdot & \cdot & \cdot & \cdot & \cdot & \cdot & \frac{1}{f g}
\end{array}\right)
$$

für $f, g \in K^{*}$. Dieser hat zur Basis

$$
\begin{aligned}
& \left\{e_{11}-b \cdot e_{12} \boldsymbol{a}, e_{22}+b \cdot e_{12} \boldsymbol{a}, e_{12}, e_{21}-b \cdot e_{11} \boldsymbol{a},\right. \\
& \left.\quad e_{11} \boldsymbol{a}, e_{22} \boldsymbol{a}+b \cdot e_{12}, e_{12} \boldsymbol{a}, e_{21} \boldsymbol{a}-b \cdot e_{11}+b \cdot e_{22}+b^{2} \cdot e_{12} \boldsymbol{a}\right\}
\end{aligned}
$$

Diagonalgestalt.

Der Schnittbereich des zugehörigen Apartments ergibt sich aus der Bedingung $r+s \leq v(b)$.

- Zur Wurzel $-\beta$ bzw. zum Charakter $[r]$ : Konjugation des Standardtorus mit

$$
\left(\begin{array}{rrrrrrrr}
1 & \cdot & \cdot & b & \cdot & \cdot & \cdot & \cdot \\
\cdot & 1 & \cdot & -b & \cdot & \cdot & \cdot & \cdot \\
-b & b & 1 & -b^{2} & \cdot & \cdot & \cdot & \cdot \\
\cdot & \cdot & \cdot & 1 & \cdot & \cdot & \cdot & \cdot \\
\cdot & \cdot & \cdot & \cdot & 1 & \cdot & \cdot & \cdot \\
\cdot & \cdot & \cdot & \cdot & \cdot & 1 & \cdot & -b \\
\cdot & \cdot & \cdot & \cdot & -b & \cdot & 1 & \cdot \\
\cdot & \cdot & \cdot & \cdot & \cdot & \cdot & \cdot & 1
\end{array}\right)
$$

liefert den Torus aller Elemente 


$$
\left(\begin{array}{cccccccc}
1 & \cdot & \cdot & b\left(\frac{1}{f}-1\right) & \cdot & \cdot & \cdot & \cdot \\
\cdot & 1 & \cdot & -b\left(\frac{1}{f}-1\right) & \cdot & \cdot & \cdot & \cdot \\
-b(1-f) & b(1-f) & f & -b^{2}\left(\frac{1}{f}-2+f\right) & \cdot & \cdot & \cdot & \cdot \\
\cdot & \cdot & \cdot & \frac{1}{f} & \cdot & \cdot & \cdot & \cdot \\
\cdot & \cdot & \cdot & \cdot & g & \cdot & \cdot & \cdot \\
\cdot & \cdot & \cdot & \cdot & \cdot & \frac{1}{g} & \cdot & -b\left(\frac{1}{f g}-\frac{1}{g}\right) \\
\cdot & \cdot & \cdot & \cdot & -b(g-f g) & \cdot & f g & \frac{1}{f g}
\end{array}\right)
$$

für $f, g \in K^{*}$. Dieser hat zur Basis

$$
\begin{aligned}
\left\{e_{11}-b \cdot e_{12}, e_{22}+b \cdot e_{12},\right. & e_{12}, e_{21}+b \cdot e_{11}-b \cdot e_{22}-b^{2} \cdot e_{12}, \\
& \left.e_{11} \boldsymbol{a}-b \cdot e_{12} \boldsymbol{a}, e_{22} \boldsymbol{a}, e_{12} \boldsymbol{a}, e_{21} \boldsymbol{a}-b \cdot e_{22} \boldsymbol{a}\right\}
\end{aligned}
$$

Diagonalgestalt.

Der Schnittbereich des zugehörigen Apartments ergibt sich aus der Bedingung $r \leq v(b)$.

\section{A.2 Berechnung für Beispiel 8.7}

$$
\begin{aligned}
& U_{-\alpha}(c) \cdot U_{\alpha}(b) \cdot t_{f, g} \cdot U_{\alpha}^{-1}(b) \cdot U_{-\alpha}^{-1}(c)
\end{aligned}
$$

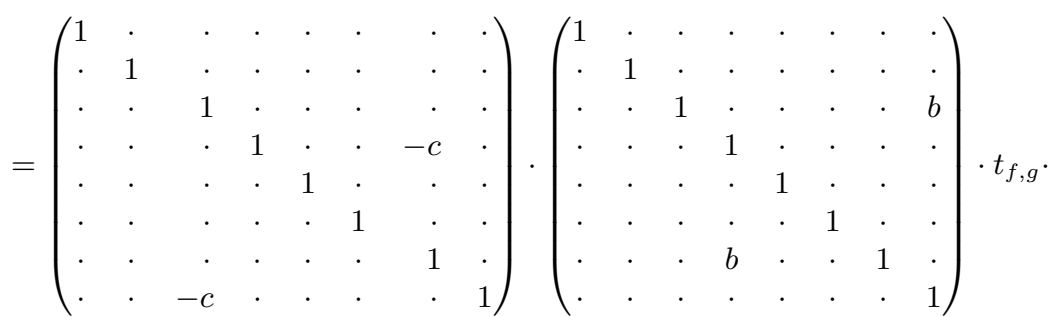

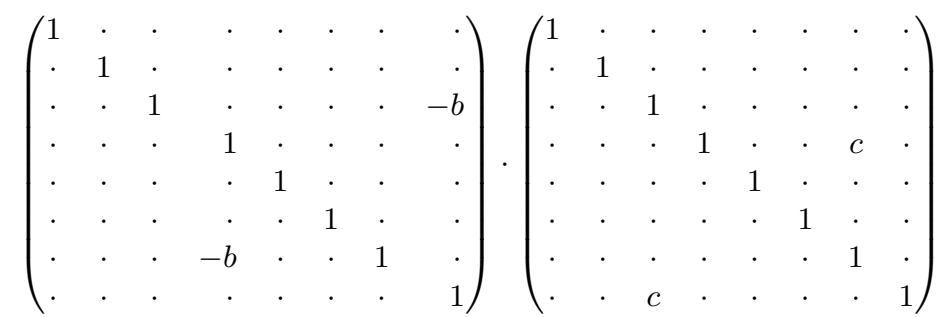

$$
\begin{aligned}
& =\left(\begin{array}{cccccccc}
\cdot & \cdot & \cdot & \cdot & \cdot & \cdot & \cdot & \cdot \\
\cdot & 1 & \cdot & \cdot & \cdot & \cdot & \cdot & \cdot \\
\cdot & \cdot & f & \cdot & \cdot & \cdot & \cdot & \frac{b}{f g} \\
\cdot & \cdot & \cdot & \frac{1-b c}{f} & \cdot & \cdot & -c f g & \cdot \\
\cdot & \cdot & \cdot & \cdot & g & \cdot & \cdot & \cdot \\
\cdot & \cdot & \cdot & \cdot & \cdot & \frac{1}{g} & \cdot & \cdot \\
\cdot & \cdot & \cdot & \frac{b}{f} & \cdot & \cdot & f g & \cdot \\
\cdot & \cdot & -c f & \cdot & \cdot & \cdot & \cdot & \frac{1-b c}{f g}
\end{array}\right) .
\end{aligned}
$$




$$
\left(\begin{array}{rccccccc}
1 & \cdot & \cdot & \cdot & \cdot & \cdot & \cdot & \cdot \\
\cdot & 1 & \cdot & \cdot & \cdot & \cdot & \cdot & \cdot \\
\cdot & \cdot & 1-b c & \cdot & \cdot & \cdot & \cdot & -b \\
\cdot & \cdot & \cdot & 1 & \cdot & \cdot & c & \cdot \\
\cdot & \cdot & \cdot & \cdot & 1 & \cdot & \cdot & \cdot \\
\cdot & \cdot & \cdot & \cdot & \cdot & 1 & \cdot & \cdot \\
\cdot & \cdot & \cdot & -b & \cdot & \cdot & 1-b c & \cdot \\
\cdot & \cdot & c & \cdot & \cdot & \cdot & \cdot & 1
\end{array}\right)=
$$

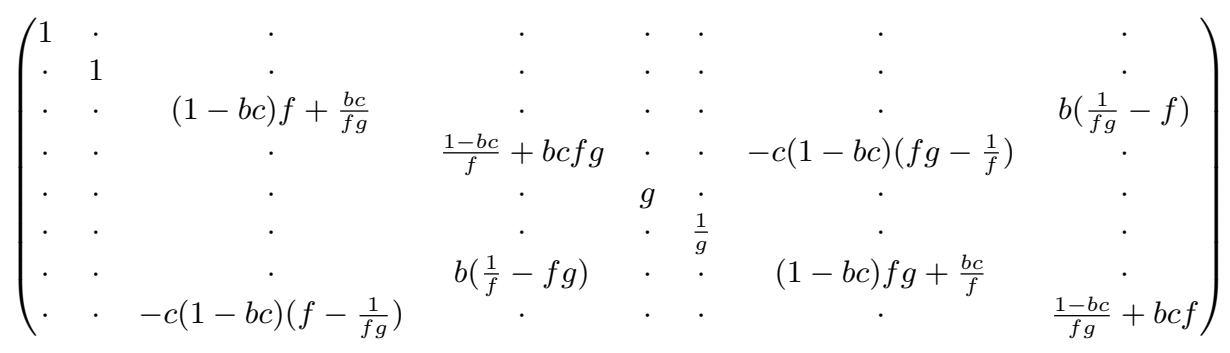

\section{A.3 Berechnung der Gleichung (10.3)}

$$
\begin{aligned}
& U_{-\beta}(p) \cdot U_{-\alpha-\beta}(q) \cdot U_{-\alpha-2 \beta}(r) \cdot U_{-\alpha-3 \beta}(s) \cdot U_{-2 \alpha-3 \beta}(t)=
\end{aligned}
$$

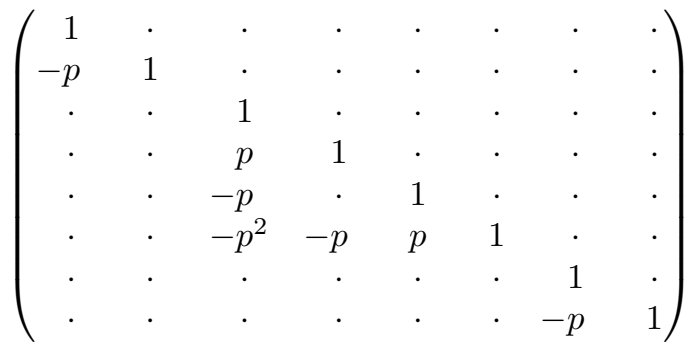

$$
\begin{aligned}
& \left(\begin{array}{cccccccc}
1 & \cdot & \cdot & \cdot & \cdot & \cdot & \cdot & \cdot \\
\cdot & 1 & \cdot & \cdot & \cdot & \cdot & \cdot & \cdot \\
q & \cdot & 1 & \cdot & \cdot & \cdot & \cdot & \cdot \\
\cdot & q & \cdot & 1 & \cdot & \cdot & \cdot & \cdot \\
\cdot & -q & \cdot & \cdot & 1 & \cdot & \cdot & \cdot \\
\cdot & \cdot & \cdot & \cdot & \cdot & 1 & \cdot & \cdot \\
\cdot & q^{2} & \cdot & q & -q & \cdot & 1 & \cdot \\
\cdot & \cdot & \cdot & \cdot & \cdot & -q & \cdot & 1
\end{array}\right) \\
& U_{-\alpha-2 \beta}(r) \cdot U_{-\alpha-3 \beta}(s) \cdot U_{-2 \alpha-3 \beta}(t) \\
& =\left(\begin{array}{cccccccc}
1 & \cdot & \cdot & \cdot & \cdot & \cdot & \cdot & \cdot \\
-p & 1 & \cdot & \cdot & \cdot & \cdot & \cdot & \cdot \\
q & \cdot & 1 & \cdot & \cdot & \cdot & \cdot & \cdot \\
p q & q & p & 1 & \cdot & \cdot & \cdot & \cdot \\
-p q & -q & -p & \cdot & 1 & . & \cdot & \cdot \\
-p^{2} q & -2 p q & -p^{2} & -p & p & 1 & \cdot & \cdot \\
\cdot & q^{2} & \cdot & q & -q & \cdot & 1 & \cdot \\
\cdot & -p q^{2} & \cdot & -p q & p q & -q & -p & 1
\end{array}\right) .
\end{aligned}
$$




$$
\begin{aligned}
& \left(\begin{array}{rrrrrrrr}
1 & \cdot & \cdot & \cdot & \cdot & \cdot & \cdot & \cdot \\
\cdot & 1 & \cdot & \cdot & \cdot & \cdot & \cdot & \cdot \\
\cdot & \cdot & 1 & \cdot & \cdot & \cdot & \cdot & \cdot \\
-r & \cdot & \cdot & 1 & \cdot & \cdot & \cdot & \cdot \\
r & \cdot & \cdot & \cdot & 1 & \cdot & \cdot & \cdot \\
\cdot & r & \cdot & \cdot & \cdot & 1 & \cdot & \cdot \\
\cdot & \cdot & r & \cdot & \cdot & \cdot & 1 & \cdot \\
-r^{2} & \cdot & \cdot & r & -r & \cdot & \cdot & 1
\end{array}\right) \cdot U_{-\alpha-3 \beta}(s) \cdot U_{-2 \alpha-3 \beta}(t) \\
& =\left(\begin{array}{cccccccc}
1 & \cdot & \cdot & \cdot & \cdot & \cdot & \cdot & \cdot \\
-p & 1 & \cdot & \cdot & \cdot & \cdot & \cdot & \cdot \\
q & \cdot & 1 & \cdot & \cdot & \cdot & \cdot & \cdot \\
p q-r & q & p & 1 & \cdot & \cdot & \cdot & \cdot \\
-p q+r & -q & -p & \cdot & 1 & \cdot & \cdot & \cdot \\
-p^{2} q+2 p r & -2 p q+r & -p^{2} & -p & p & 1 & \cdot & \cdot \\
-2 q r & q^{2} & r & q & -q & \cdot & 1 & \cdot \\
2 p q r-r^{2} & -p q^{2}-q r & -p r & -p q+r & p q-r & -q & -p & 1
\end{array}\right) . \\
& \left(\begin{array}{cccccccc}
1 & \cdot & \cdot & \cdot & \cdot & \cdot & \cdot & \cdot \\
\cdot & 1 & \cdot & \cdot & \cdot & \cdot & \cdot & \cdot \\
\cdot & \cdot & 1 & \cdot & \cdot & \cdot & \cdot & \cdot \\
\cdot & \cdot & \cdot & 1 & \cdot & \cdot & \cdot & \cdot \\
\cdot & \cdot & \cdot & \cdot & 1 & \cdot & \cdot & \cdot \\
-s & \cdot & \cdot & \cdot & \cdot & 1 & \cdot & \cdot \\
\cdot & \cdot & \cdot & \cdot & \cdot & \cdot & 1 & \cdot \\
\cdot & \cdot & \cdot & \cdot & \cdot & \cdot & \cdot & 1
\end{array}\right) \cdot U_{-2 \alpha-3 \beta}(t) \\
& =\left(\begin{array}{cccccccc}
1 & \cdot & \cdot & \cdot & \cdot & \cdot & \cdot & \cdot \\
-p & 1 & \cdot & \cdot & \cdot & \cdot & \cdot & \cdot \\
q & \cdot & 1 & \cdot & \cdot & \cdot & \cdot & \cdot \\
p q-r & q & p & 1 & \cdot & \cdot & \cdot & \cdot \\
-p q+r & -q & -p & \cdot & 1 & \cdot & \cdot & \cdot \\
-p^{2} q+2 p r-s & -2 p q+r & -p^{2} & -p & p & 1 & \cdot & \cdot \\
-2 q r & q^{2} & r & q & -q & \cdot & 1 & \cdot \\
2 p q r-r^{2}+q s & -p q^{2}-q r & -p r+s & -p q+r & p q-r & -q & -p & 1
\end{array}\right) \\
& \left(\begin{array}{cccccccc}
1 & \cdot & \cdot & \cdot & \cdot & \cdot & \cdot & \cdot \\
\cdot & 1 & \cdot & \cdot & \cdot & \cdot & \cdot & \cdot \\
\cdot & \cdot & 1 & \cdot & \cdot & \cdot & \cdot & \cdot \\
\cdot & \cdot & \cdot & 1 & \cdot & \cdot & \cdot & \cdot \\
\cdot & \cdot & \cdot & \cdot & 1 & \cdot & \cdot & \cdot \\
\cdot & \cdot & \cdot & \cdot & \cdot & 1 & \cdot & \cdot \\
-t & \cdot & \cdot & \cdot & \cdot & \cdot & 1 & \cdot \\
\cdot & -t & \cdot & \cdot & \cdot & \cdot & \cdot & 1
\end{array}\right)
\end{aligned}
$$




$$
=\left(\begin{array}{cccccccc}
1 & \cdot & \cdot & \cdot & \cdot & \cdot & \cdot & \cdot \\
-p & 1 & \cdot & \cdot & \cdot & \cdot & \cdot & \cdot \\
q & \cdot & 1 & \cdot & \cdot & \cdot & \cdot & \cdot \\
p q-r & q & p & 1 & \cdot & \cdot & \cdot & . \\
-p q+r & -q & -p & \cdot & 1 & \cdot & \cdot & \cdot \\
-p^{2} q+2 p r-s & -2 p q+r & -p^{2} & -p & p & 1 & \cdot & . \\
-2 q r-t & q^{2} & r & q & -q & \cdot & 1 & . \\
2 p q r-r^{2}+q s+p t & -p q^{2}-q r-t & -p r+s & -p q+r & p q-r & -q & -p & 1
\end{array}\right)
$$




\section{Literatur}

[Bae02] Baez, John C.: The octonions. Bull. Amer. Math. Soc. (N.S.), 39(2):145-205, 2002.

[Beh95] Behrend, Kai A.: Semi-stability of reductive group schemes over curves. Math. Ann., 301(2):281-305, 1995.

[Bor91] Borel, Armand: Linear algebraic groups, Band 126 der Reihe Graduate Texts in Mathematics. Springer-Verlag, New York, 2. Auflage, 1991.

[GS06] Gille, Philippe und Szamuely, Tamás: Central simple algebras and Galois cohomology, Band 101 der Reihe Cambridge Studies in Advanced Mathematics. Cambridge University Press, Cambridge, 2006.

[GY03] Gan, Wee Teck und Yu, Jiu Kang: Schémas en groupes et immeubles des groupes exceptionnels sur un corps local. I. Le groupe $G_{2}$. Bull. Soc. Math. France, 131(3):307-358, 2003.

[HS03] Harder, Günter und Stuhler, Ulrich: Canonical parabolic subgroups of Arakelov group schemes. [http://www.unimath.gwdg.de/stuhler/arakelov.pdf, Preprint, 2003.

[Ker07] Kersten, Ina: Lineare algebraische Gruppen. Universitätsdrucke Göttingen. Göttingen: Universitätsverlag., 2007.

[Kne02] Kneser, Martin: Quadratische Formen. Neu bearbeitet und herausgegeben in Zusammenarbeit mit Rudolf Scharlau. Springer-Verlag, Berlin, 2002.

[Lan96] Landvogt, Erasmus: A compactification of the Bruhat-Tits building, Band 1619 der Reihe Lecture Notes in Mathematics. SpringerVerlag, Berlin, 1996.

[Spr63] Springer, Tonny A.: Oktaven, Jordan-Algebren und Ausnahmegruppen. Mathematisches Institut der Universität Göttingen, Göttingen, 1963.

[Str02] Stroth, Kristin: Quaternionenalgebren über rationalen Funktionenkörpern in zwei Unbestimmten. Diplomarbeit, Georg-August-Universität Göttingen, 2002.

[SV00] Springer, Tonny A. und Veldkamp, Ferdinand D.: Octonions, Jordan algebras and exceptional groups. Springer Monographs in Mathematics. Springer-Verlag, Berlin, 2000. 
[Tit79] Tits, J.: Reductive groups over local fields. In: Automorphic forms, representations and L-functions (Proc. Sympos. Pure Math., Oregon State Univ., Corvallis, Ore., 1977), Part 1, Proc. Sympos. Pure Math., XXXIII, Seiten 29-69. Amer. Math. Soc., Providence, R.I., 1979 .

[Wat79] Waterhouse, William C.: Introduction to affine group schemes, Band 66 der Reihe Graduate Texts in Mathematics. SpringerVerlag, New York, 1979. 


\section{Index}

$\alpha, 42$

$\beta, 42$

$a, 10$

$\pi, 49$

$\sigma, 32$

$\tau, 32$

affine Weylgruppe, 42

affines Gruppenschema, 12

Apartment, 40

Arakelov-Bündel, 18

Arakelov-Geradenbündel, 18

Grad, 19

Bewertung, 46

Bruhat-Tits-Gebäude, 40

Cartan-Killing-Form, 58

Cayley-Dickson-Verfahren, 7

Cayley-Zahlen, 5

darstellbarer Funktor, 12

Determinanten-Geradenbündel, 18

dominantes Gewicht, 25

duale Polyeder, 28

Ecke, 26

exakte Sequenz, 19

Facette, 22

fundamental dominantes Gewicht, 25

fundamentales Gewicht, 25

Funktor

darstellbarer, 12

natürlicher, 13

Gewicht, 24

dominantes, 25

fundamental dominantes, 25

fundamentales, 25

Gitter

der Gewichte, 24

der Kogewichte, 24
Grad, 19, 29

Hamiltonsche Quaternionen, 9

Harder-Narasimhan-Filtration, 21

HN-Filtration, 21

Homomorphismus

von Arakelov-Bündeln, 18

Hopf-Algebra, 15

inneres Produkt, 6

invariant, 58

Ko-Charaktere des Torus, 40

Koeins, 14

Kogewichte, 24

kohärente Normen, 17

Koinverses, 14

Komplementärpolyeder, 28

Komposition, 6

Kompositions-Unteralgebra, 6

Kompositionsalgebra, 6

über Ringen, 7

Unteralgebra, 6

zerfallende, 9

Komultiplikation, 14

Konjugation, 6

Kowurzeln, 22

Lemma

von Yoneda, 13

Modul

nichtsingulärer, 5

natürlicher Funktor, 13

negative Wurzeln, 25

nichtausgeartet, 5

nichtsingulär, 5

Norm, 6, 16

kohärente, 17

normierter Vektorraum, 16

Oktav-Algebra, 9 
Oktaven, 5, 9

Oktonionen, 9

Oktonionen-Algebra, 9

positive Wurzeln, 25

Produktformel, 16

Projektion, 27

quadratische Form, 5 nicht ausgeartete, 5

Quaternionen, 9

Hamiltonsche, 9

Quaternionenalgebra, 9

Quotientenbündel, 19

Reduktion, 27

reduziertes Wurzelsystem, 22

renormalisiert, 61 .

semistabil, 20, 30

spaltende Basis, 46

spezielle Punkte, 44

stabil, 20

Standard-Involution, 58

Standard-Torus, 31

Standardflagge, 67

Steigung, 20

symmetrischer Raum, 60

uniformisierendes Element, 49

Verdopplungsverfahren, 7

Weylgruppe, 33

affine, 42

Wurzeln, 22

negative, 25

positive, 25

Wurzelsystem, 22

Yoneda-Lemma, 13

zerfallend, 9

Zimmer, 22 


\title{
Lebenslauf
}

\section{Karsten Roeseler}

\author{
6. März 1975 Geboren in Hamburg \\ ledig, keine Kinder
}

\section{Ausbildung}

1981-1985

Carl-Orff-Schule in Bargteheide

1985-1994

Kreisgymnasium Bargteheide

$07 / 1994$

Abitur am Kreisgymnasium Bargteheide Leistungsfächer: Mathematik, Chemie

08/1994-11/1995 Zivildienst beim Deutschen Roten Kreuz (mobiler sozialer Hilfsdienst) in Bargteheide

10/1995 Beginn des Studiums der Mathematik an der Universität Hamburg

10/1995-12/2000 Stipendiat der Studienstiftung des deutschen Volkes

09/1997 Vordiplom in Mathematik an der Universität Hamburg; anschließend Studienortwechsel nach Göttingen

02/2002 Diplom in Mathematik am Mathematischen Institut der Georg-August-Universität Göttingen;

Betreuer der Diplomarbeit: Prof. Dr. Stuhler.

Seit 03/2002 Promotionsstudium am Mathematischen Institut der GeorgAugust-Universität Göttingen, Betreuer: Prof. Dr. Stuhler

03/2002-02/2005 Stipendiat der DFG im Graduiertenkolleg „Gruppen und Geometrie"

Göttingen, den 14. Januar 2011 